\title{
Analysis of peroxisomal turnover and myelin maintenance in mice with oligodendrocyte-specific MFP2-deficiency
}

\author{
Dissertation \\ For the award of the degree \\ "Doctor rerum naturalium" (Dr. rer. nat.) \\ of the Georg-August University Göttingen \\ within the doctoral program Biology \\ of the Georg-August University School of Science (GAUSS)
}

submitted by

\author{
Sarah Richert \\ from Celle, Germany
}

Göttingen,

2016 


\section{Examination board}

Prof. Klaus-Armin Nave Ph.D. (Reviewer)

Department of Neurogenetics

Max Planck Institute of Experimental Medicine, Göttingen

Prof. Dr. Ernst A. Wimmer (Reviewer)

Department of Developmental Biology

Johann-Friedrich-Blumenbach-Institute of Zoology and Anthropology (GZMB)

Georg-August University, Göttingen

Priv.-Doz. Dr. Sven Thoms

Department of Pediatrics and Pediatric Neurology

University Medical Center, Göttingen

Prof. Dr. Volker Lipka

Schwann-Schleiden Research Centre

Department of Plant Cell Biology

Georg-August University, Göttingen

Prof. Dr. Peter Schu

Department of Cellular Biochemistry

University Medical Center, Göttingen

Prof. Dr. Ralf Heinrich

Department of Neurobiology

Institute for Zoology and Anthropology

Georg-August University, Göttingen

Date of oral examination: 17.10.2016 


\section{Declaration}

I hereby declare that the $\mathrm{PhD}$ thesis entitled 'Analysis of peroxisomal turnover and myelin maintenance in mice with oligodendrocyte-specific MFP2-deficiency' has been written independently, with no other aids or sources than quoted.

Göttingen,

Sarah Richert 


\section{Table of contents}

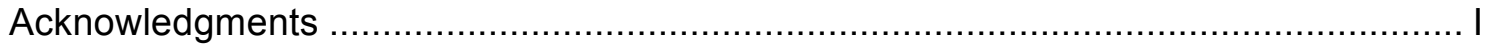

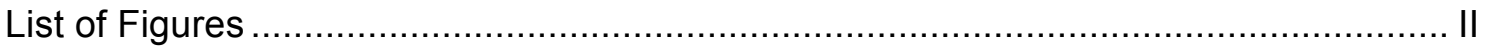

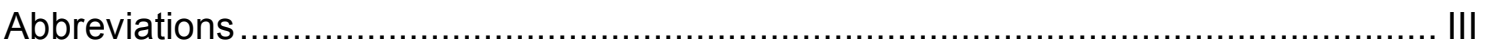

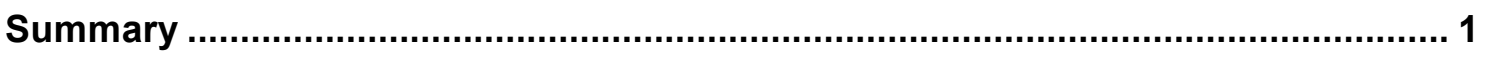

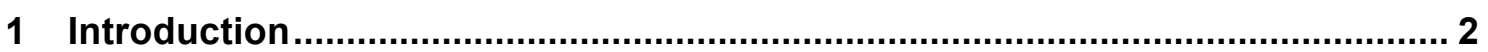

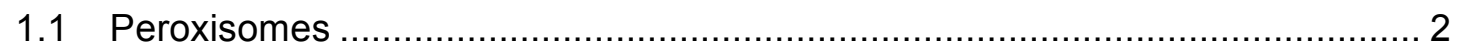

1.1.1 Peroxisomal metabolism and its biological importance ........................... 2

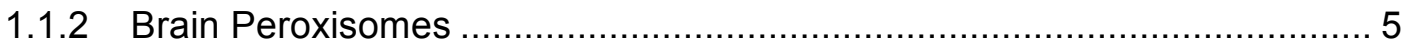

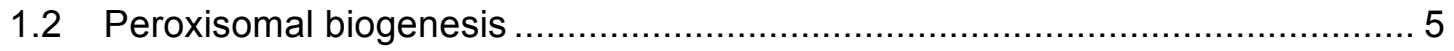

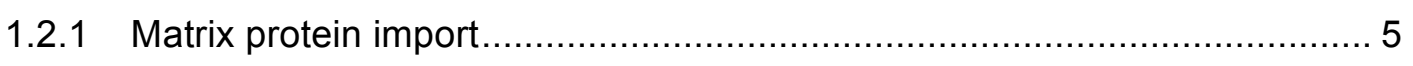

1.2.2 Insertion of peroxisomal membrane proteins ...................................... 7

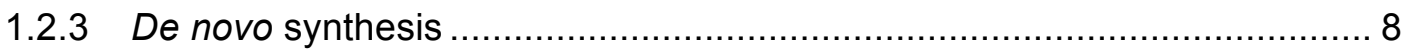

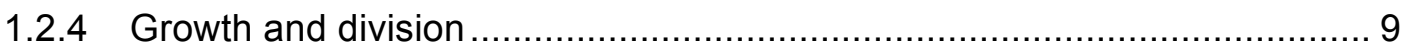

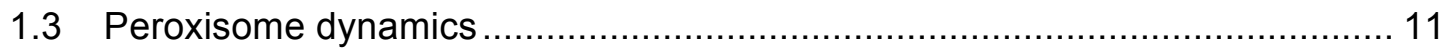

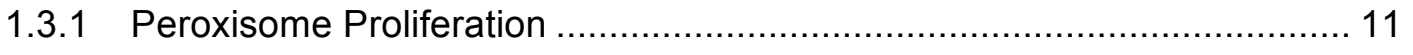

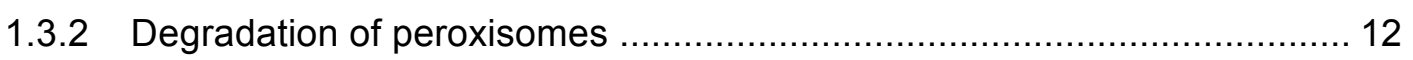

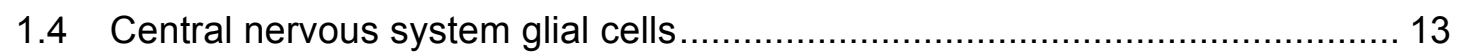

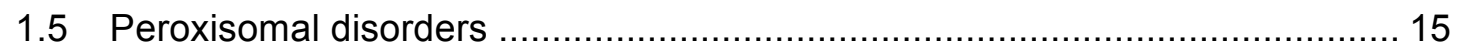

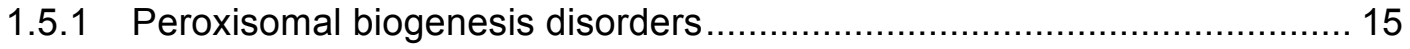

1.5.2 Single peroxisome transporter and enzyme deficiencies ....................... 16

1.5.3 Mouse models of peroxisomal diseases ........................................... 17

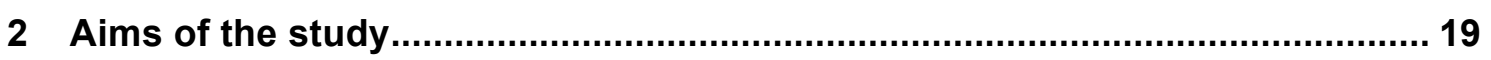

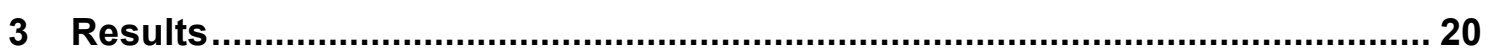

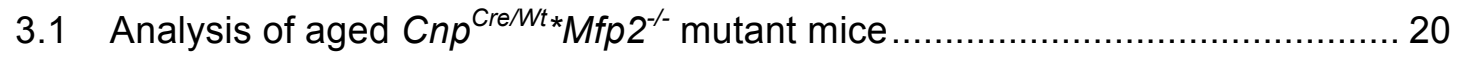

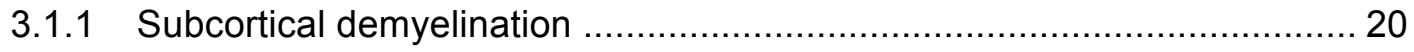

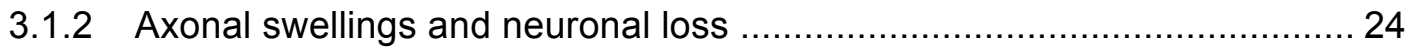

3.1.3 Reactive gliosis in the frontal corpus callosum ..................................... 25

3.1.4 T-lymphocyte infiltration in sites of demyelination ................................. 27

3.2 Generation and analysis of Cnp-mEos2-SKL transgenic mice ...................... 28

3.2.1 Generation of Cnp-mEos2-PTS1 transgenic mice ................................ 28

3.2.2 Spatio-temporal expression pattern of Cnp-mEos2-PTS1 ...................... 29

3.2.3 Peroxisomal targeting of mEos2-SKL ................................................... 32

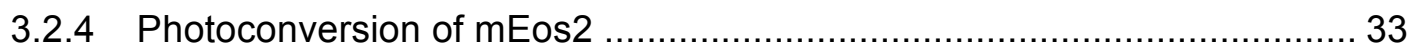


3.2.5 Cnp-mEos2-PTS1 expression in oligodendrocytes is not toxic

3.3 Reduced peroxisomal abundance and slowed peroxisomal turn-over in $\operatorname{tg} \mathrm{Cnp}^{\mathrm{Cre} / \mathrm{Wt} *} \mathrm{Mfp} 2^{-/}$mice

3.3.1 Reduced peroxisomal abundance in $\operatorname{tgCnp}{ }^{\mathrm{Cre} / W t \star M f p 2^{-/}}$mice ................... 37

3.3.2 $\operatorname{tgAbcd1/}$ mutants do not exhibit decreased peroxisome abundance ....... 40

3.3.3 MFP2-deficient peroxisomes differ in shape and size ............................ 41

3.3.4 Peroxisomal degradation and proliferation in oligodendrocytes ............... 42

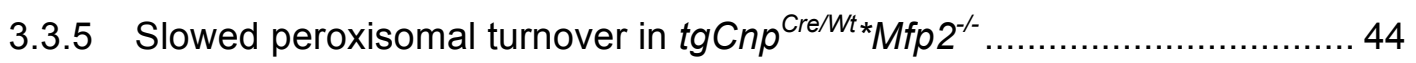

3.3.6 3-Methyladenine treatment reduces autophagic degradation and

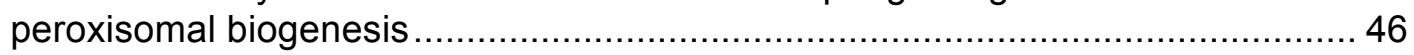

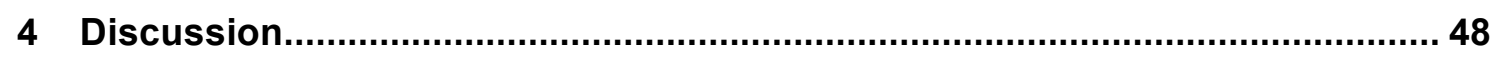

4.1 Cnp ${ }^{\mathrm{Cre} / \mathrm{Wt}_{*}} \mathrm{Mfp}^{-{ }^{-/}}$display a late-onset subcortical demyelination reminiscent of mice with peroxisomal biogenesis defects in oligodendrocytes.

4.2 Cnp-mEos2-PTS1 mice enable cell type-specific analysis of peroxisomes..... 52

4.3 Progressive loss of oligodendroglial peroxisomes in $\operatorname{tgCnp}{ }^{\mathrm{Cre} / \mathrm{Wt} *} \mathrm{Mfp}^{-/-}$mutants,

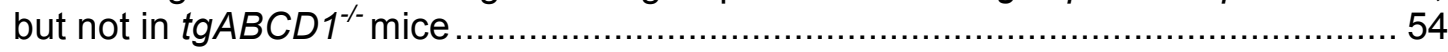

4.4 MFP2-deficiency decreases peroxisomal turnover in oligodendrocytes .......... 56

4.4.3 Direct effects of beta-oxidation impairment on fission? ............................ 57

4.4.4 Direct effects of beta-oxidation impairment on autophagy? ...................... 58

4.5 Heterozygosity is not responsible for the pathology of $\mathrm{Cnp}^{\mathrm{Cre} / \mathrm{Wt}{ }_{*}} \mathrm{Mfp} 2^{-/-}$ mutants..

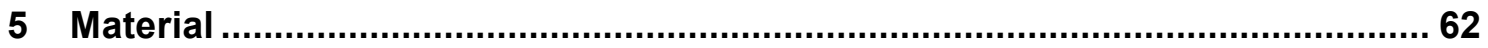

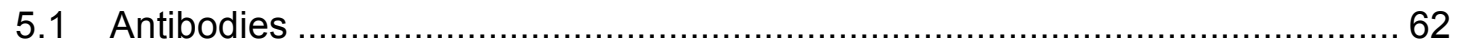

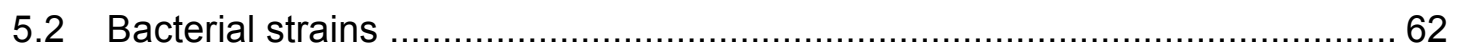

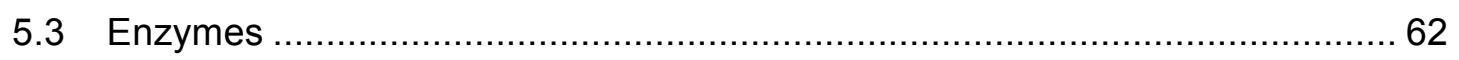

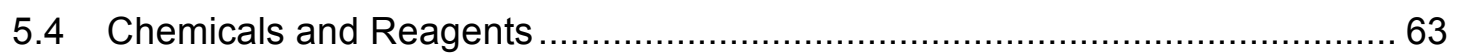

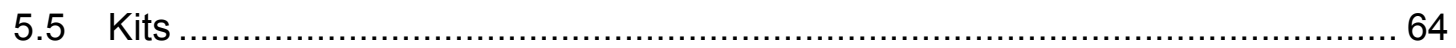

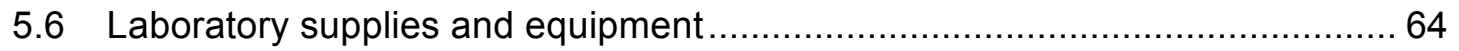

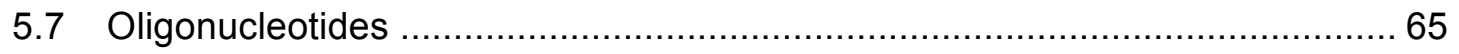

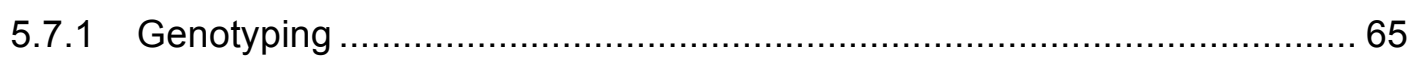

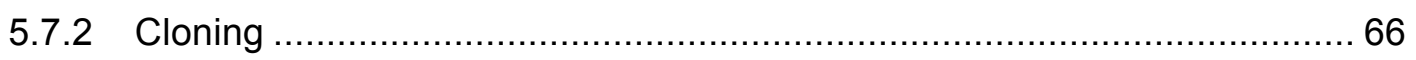

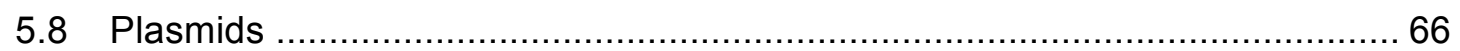

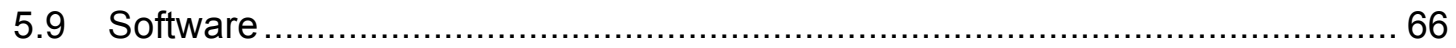

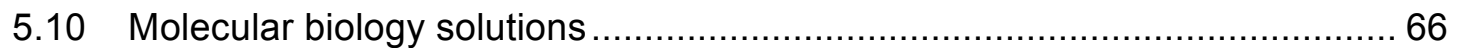

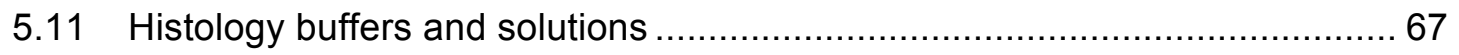

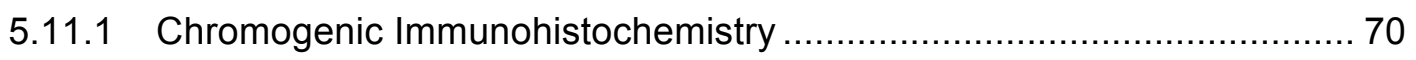

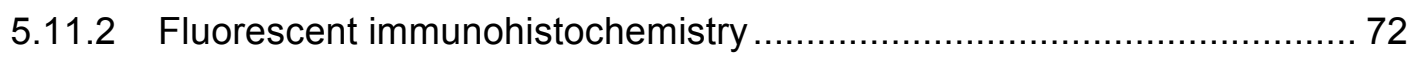




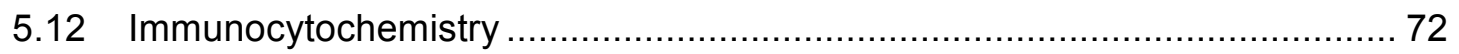

5.13 Solutions and media for primary oligodendrocytes .................................. 73

5.14 Solutions for genomic DNA preparation................................................ 73

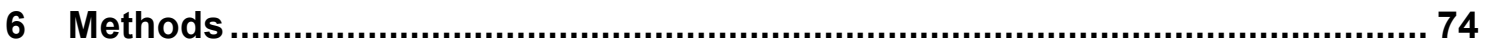

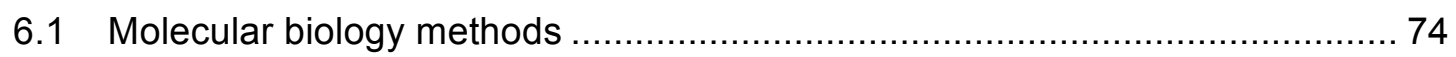

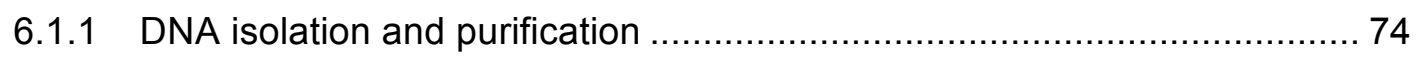

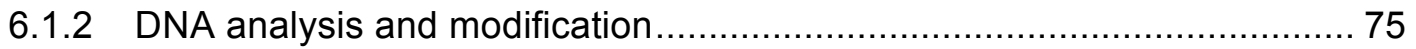

6.1.3 Transformation of chemically competent bacteria ................................ 78

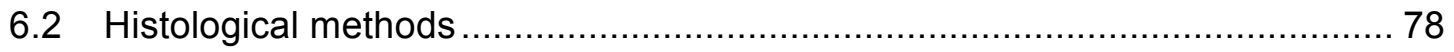

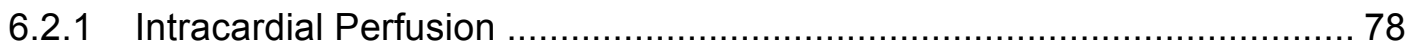

6.2.2 Procedures for tissue embedding and sectioning .............................. 79

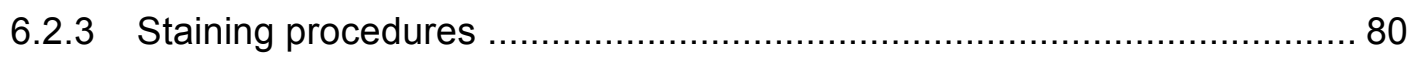

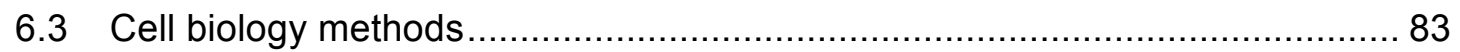

6.3.1 Preparation and culture of primary oligodendrocytes ............................ 83

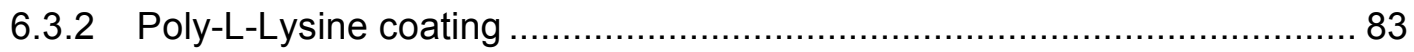

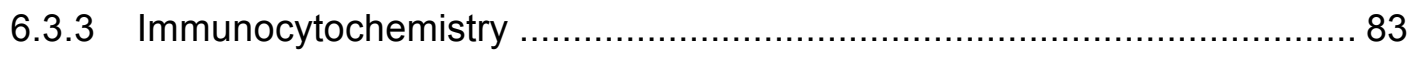

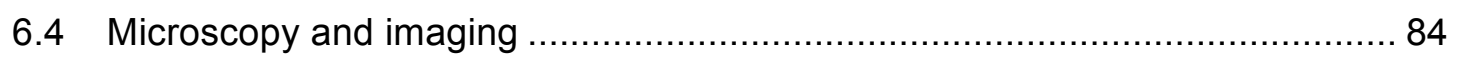

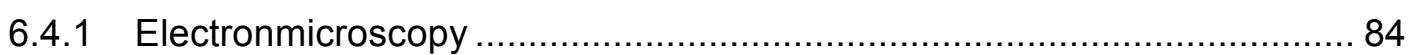

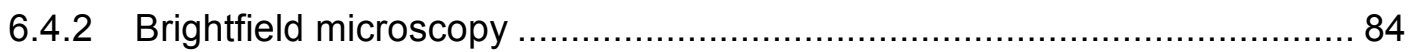

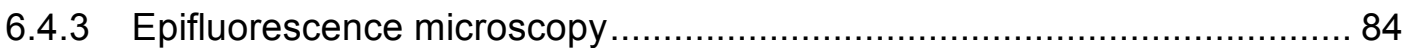

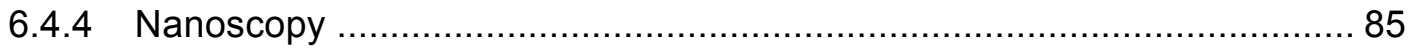

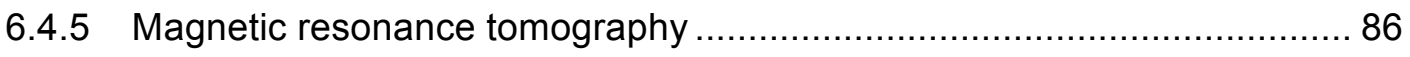

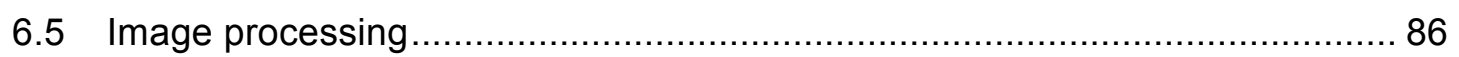

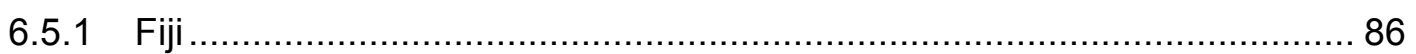

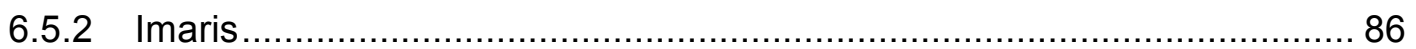

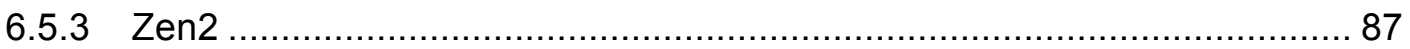

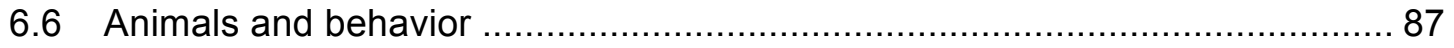

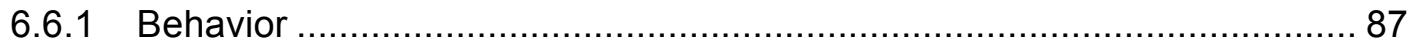

6.6.2 Generation of transgenic Cnp-mEos2-PTS1 mice .............................. 88

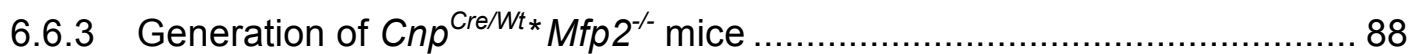

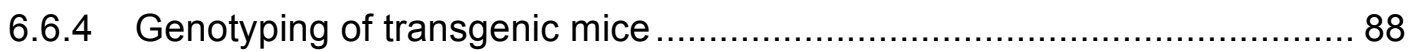

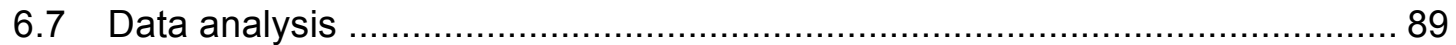

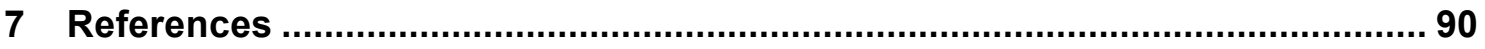

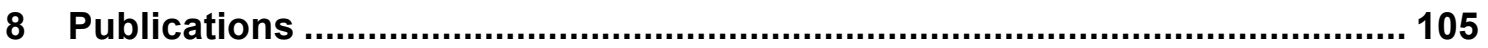




\section{Acknowledgments}

I would like to express my sincere gratitude and appreciation to my supervisor Dr. Celia Kassmann, whose positive attitude and dedication to science greatly motivated and inspired me. I feel grateful for the many stimulating discussions, your support and guidance.

I thank Prof. Klaus-Armin Nave for the opportunity to work in his department, which provided a great working environment. Thank you for sharing your scientific experience and for interesting discussions.

My thanks also go to my thesis committee members, Prof. Dr. Ernst A. Wimmer and Priv.-Doz. Dr. Sven Thoms for fruitful discussions, advices and support during the thesis. Furthermore, I want to appreciate the participation of Prof. Dr. Volker Lipka, Prof. Dr. Peter Schu and Prof. Dr. Ralf Heinrich in the defense.

\section{Many Thanks also to....}

... the whole Kassmann Group, especially Jenni Günther. I'm extremely grateful for all the help throughout the years and also for your friendship.

... Michaela Schmalstieg and Gabriele Endo for your care and support.

... Ulli Bode for technical help in many ways, but also for making everyday life more fun.

... Dr. Wiebke Möbius, Torben Ruhwedel and Boguslawa Sadowski for everything related to electron microscopy.

... Annette Fahrenholz for support with immunohistology.

... Hajo Horn, Beate Beschke and Lothar Demel for support with computer issues.

... the animal caretakers for their great work.

... Prof. Myriam Baes for collaboration and providing mice.

... Prof. Gerd Ulrich Nienhaus for providing the mEos2 construct.

... Dr. Katrin Willig for enabling STED analysis.

... Dr. Thomas Michaelis for the MRI analysis of our mice.

... my girls, Hannah, Lotti, Resi, and Uli for the great times we spent inside and outside the lab. For your help, support, and always being there. This joint experience will unite us for the rest of our lives.

... My roommate Jule, who went with me through the same ups and downs of a PhD thesis. Thank you for your open ear when I needed it, for your relaxing attitude and the great times we spent together.

... Luis, Mona and my family, especially my mother, grandmother and brothers, who always had an open ear for me and supported and encouraged me even though sometimes they would have to endure endless monologues on peroxisomes. 


\section{List of Figures}

Fig. I: A schematic view of main metabolic pathways in peroxisomes ....................... 4

Fig. II: Import of peroxisomal proteins containing a PTS1 .................................... 7

Fig. III: Schematic representation of peroxisome proliferation ................................ 10

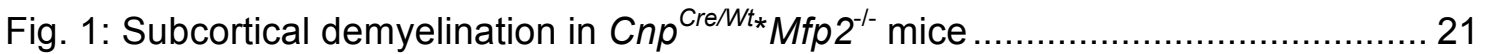

Fig. 2: Clinical symptoms and sings for ataxia in $\geq 16$-month-old $C n p^{\mathrm{Cre} / W t *} \mathrm{Mfp}^{-/-}$

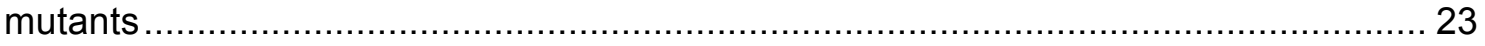

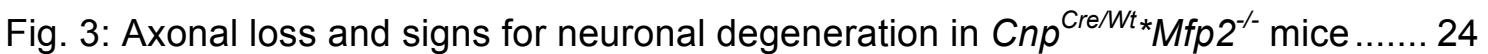

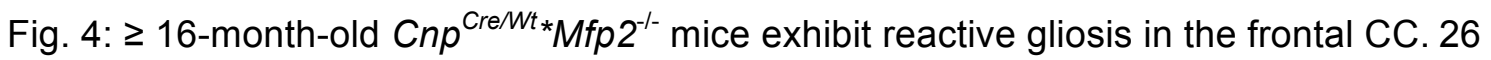

Fig. 5: T-lymphocyte infiltration in the anterior $\mathrm{CC}$ in $\mathrm{Cnp}^{\mathrm{Cre} / W{ }_{*}} \mathrm{Mfp}^{-{ }^{-/}}$mice .................. 27

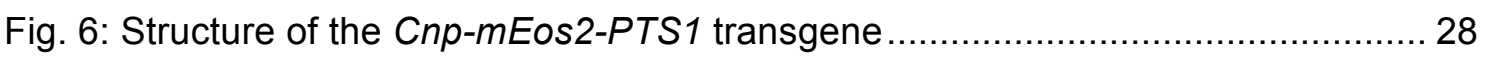

Fig. 7: Expression of mEos2 in the CNS of Cnp-mEos2-PTS1 transgenic mice .......... 30

Fig. 8: The majority of Olig2+ cells in Cnp-mEos2-PTS1 transgenic mice show

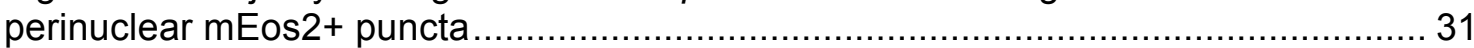

Fig. 9: mEos2+ puncta are absent from astrocytes, microglia, and neurons .............. 32

Fig. 10: mEos2 targets exclusively to peroxisomes in Cnp-mEos2-PTS1 mice.........33

Fig. 11: 2D contour plot from a $\lambda$ scan of Cnp-mEos2-PTS1 transfected cells confirms

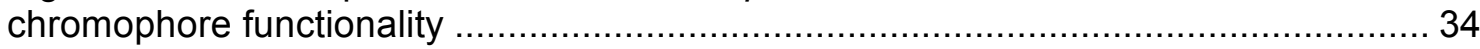

Fig.12: Photoconversion of live cells expressing mEos2-PTS1 …........................... 35

Fig. 13: Photoconversion in different regions of vibratome sections from Cnp-mEos2-

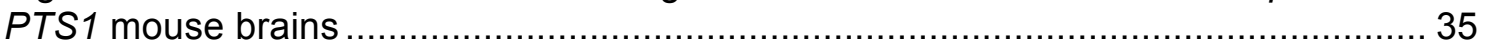

Fig.14: 12-month-old Cnp-mEos2-PTS1 mice show no signs of reactive gliosis ......... 36

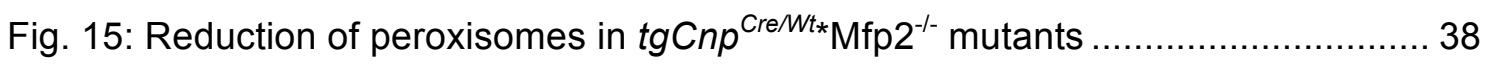

Fig. 16: Reduction of peroxisomes in areas without overt pathology .......................... 39

Fig. 17: Peroxisome reduction is not a feature of $\operatorname{tg} A b c d 1^{-/}$mutants ....................... 40

Fig. 18: STED microscopy confirms increased size and altered shape of

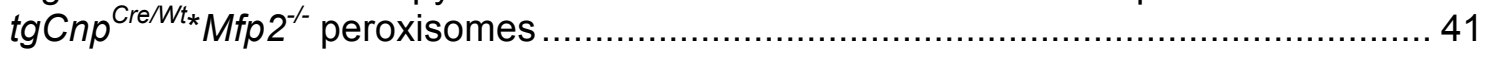

Fig. 19: Peroxisomal turnover in CTR oligodendrocytes .......................................... 43

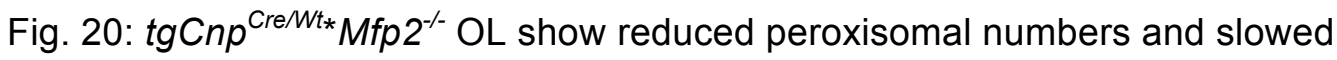

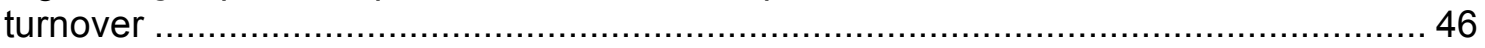

Fig. 21: Reduced autophagic degradation also impairs peroxisomal biogenesis ......... 47 


\section{Abbreviations}

ABCD

ACAA1

ACOX1/2

AGPS

ALD

AMACR

AMN

AmpR

ATP

AU

BCFA

BSA

CAT

CC

CCALD

cDNA

cKO

CMV

CNP

CNS

DAB

DBP

DMEM

DMSO

DNA

DNase

dNTP

DTT

E.coli

EDTA

EGFP

et al.

$\mathrm{EtOH}$

FA

FBS
ATP-binding cassette protein subtype D

3-ketoacyl-CoA thiolase,

Acyl-CoA oxidase 1/2

Alkyl-dihydroxyacetonephosphate synthase,

Adrenoleukodystrophy

2-methylacyl-CoA racemace,

Adrenomyeloneuropathy

Ampicillin resistance

Adenosine triphosphate

Arbitrary units

Branched-chain FA

Bovine serum albumin

Catalase

Corpus Callosum

Childhood cerebral ALD

Complementary DNA

Conditional Knock-out mouse

Cytomegalovirus

$2,3^{\prime}, 3$-cyclic nucleotide phosphodiesterase

Central nervous system

3,3'Diaminobenzidine

D-bifunctional protein

Dulbecco's modified Eagle Medium

Dimethyl sulfoxide

Deoxyribonucleic acid

Deoxyribonuclease

Deoxyribonucleotide triphosphate

1,4-Dithiothreitol

Escherichia coli

Ethylendiamintetraacetate

Enhanced Green Fluorescent Protein

And others

Ethanol

Fatty acid

Fetal Bovine Serum 


\begin{tabular}{|c|c|}
\hline GFAP & Glial fibrillary acidic protein \\
\hline GNPAT & Dihydroxyacetonephosphate acyltransferase, \\
\hline HBSS & Hank's buffered salt solution \\
\hline HE & Haematoxylin-Eosin \\
\hline HS & Horse Serum \\
\hline IHC & Immunohistochemistry \\
\hline KanR & Kanamycin resistance \\
\hline $\mathrm{KO}$ & Knock-out mouse \\
\hline LB & Luria and Bertani medium (or Luria's broth) \\
\hline LBP & L-bifunctional protein \\
\hline $\mathrm{LCl}$ & Live cell imaging \\
\hline LFB & Luxol fast blue \\
\hline MBP & Myelin basic protein \\
\hline MFP2 & Multifunctional protein 2 \\
\hline mRNA & Messenger-RNA \\
\hline ON & Overnight \\
\hline PBS & Phosphate-buffered Saline \\
\hline PCR & Polymerase Chain Reaction \\
\hline Pex & Peroxin \\
\hline PFA & Paraformaldehyde \\
\hline $\mathrm{pH}$ & Negative decimal logarithm of hydrogen ions \\
\hline PLL & Poly-L-Lysin \\
\hline PMP & Peroxisomal membrane Protein \\
\hline PNS & Peripheral nervous system \\
\hline Pex & Peroxin \\
\hline qRT-PCR & Quantitative real-time PCR \\
\hline RNA & Ribonucleic acid \\
\hline RNase & Ribonuclease \\
\hline RT & Room Temperature \\
\hline SCPX & Sterol carrier protein $X$ \\
\hline SDS & Sodium Dodecyl Sulfate \\
\hline SV40 & Simian Virus 40 \\
\hline Taq & Thermus aquaticus \\
\hline TBS & Tris buffered saline \\
\hline $\operatorname{Tg}$ & Transgenic \\
\hline $\mathrm{Tm}$ & Melting temperature \\
\hline
\end{tabular}


UV

VLCFA

WT

$X-A L D$

ZSS
Ultraviolet

Very long chain fatty acids

Wild type

X-linked Adrenoleukodystrophy

Zellweger syndrome spectrum

\section{Units of measurement}

$\begin{array}{ll}\mathrm{g} & \text { Gram } \\ \mathrm{b} & \text { Bases } \\ \mathrm{bp} & \text { Base pairs } \\ { }^{\circ} \mathrm{C} & \text { Degrees Celsius } \\ \mathrm{h} & \text { Hour } \\ \mathrm{kb} & \text { Kilobase } \\ \mathrm{M} & \text { Molar } \\ \mathrm{mA} & \text { Milliampere } \\ \mathrm{min} & \text { Minute } \\ \mathrm{n} & \text { Number } \\ \mathrm{OD} & \text { Optical density } \\ \mathrm{rpm} & \text { Rotation per minute } \\ \mathrm{sec} & \text { Seconds } \\ \mathrm{U} & \text { Units of enzymatic activity } \\ \mathrm{V} & \text { Volt } \\ \mathrm{w} / \mathrm{V} & \text { Weight / volume }\end{array}$

Power prefixes:

$\begin{array}{ll}\mathrm{m} & \text { milli }\left(10^{-3}\right) \\ \mu & \text { micro }\left(10^{-6}\right) \\ \mathrm{n} & \text { nano }\left(10^{-9}\right)\end{array}$




\section{Summary}

Although caused by distinct genetic mutations, the peroxisomal disorders X-linked adrenoleukodystrophy (X-ALD), pseudoneonatal adrenoleukodystrophy (pseudo NALD), and neonatal adrenoleukodystrophy (NALD) share several disease hallmarks. Strikingly, the underlying dysfunctions are either related to generalized peroxisomal defects or to defects in peroxisomal $\beta$-oxidation. In contrast, leukodystrophy is not a feature of other peroxisomal disorders e.g. in $\alpha$-oxidation or plasmalogen-synthesis defects. This indicates a common pathomechanism for $\beta$-oxidation and generalized peroxisomal defects. Further indications for this derive from mice lacking complete peroxisomal function only in oligodendrocytes. The pattern of inflammatory subcortical demyelination in these $\mathrm{Cnp}{ }^{\mathrm{Cre} / \mathrm{Wt} *} \mathrm{Pex} 5^{-/}$mutants is reminiscent of the cerebral pathology known from X-ALD patients lacking peroxisomal $\beta$-oxidation of very long chain fatty acids. This led to the hypothesis that impaired $\beta$-oxidation may culminate in secondary loss of further peroxisomal functions. So far, several mouse models with defective peroxisomal $\beta$-oxidation were generated, but failed to develop cerebral demyelination. In search for an appropriate model with impaired peroxisomal $\beta$-oxidation to investigate possible secondary peroxisomal defects and subsequent pathology, aged

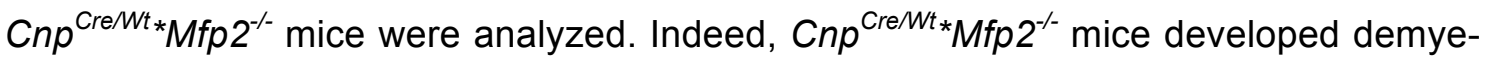
linating lesions in the frontal corpus callosum when aged $\geq 16$ months. This was accompanied by reactive gliosis, lymphocyte infiltration, and behavioral alterations. Thus

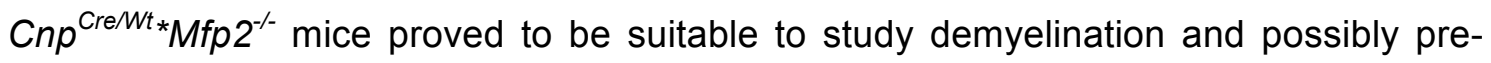
ceding effects of impaired peroxisomal $\beta$-oxidation on peroxisomes. To facilitate oligodendrocyte specific analysis of oligodendroglial peroxisomes novel transgenic mice with fluorescently labeled peroxisomes in oligodendrocytes (Cnp-mEos2-PTS1) were generated. Employing a photo-convertible fluorescent protein enabled 'pulse-chase' experiments to provide insight into peroxisomal biogenesis and degradation. Brain sections from double-transgenic Cnp-mEos2-PTS ${ }^{*} \mathrm{C} n p^{\mathrm{Cr} / \mathrm{Wt} *}{ }^{*} \mathrm{Mfp} 2^{-/-}$mice revealed a progressively decreasing number and increased size of peroxisomes. Alterations were observed already at 2 months, preceding disease onset by approximately one year. At 16 months of age only $50 \%$ of peroxisomes were preserved. In vitro experiments using primary MFP2-deficient oligodendrocytes proved a dramatically reduced peroxisomal turnover by both, decreased degradation of pulse-labeled peroxisomes, i.e. enhanced organelle aging, and diminished appearance of new peroxisomes. The mechanism of this decreased organelle turnover remains elusive. Interestingly, inhibiting pexophagy in control oligodendrocytes by use of 3-Methyladenine also blocked peroxisomal biogenesis, which indicates tight coupling between peroxisomal biogenesis and degradation. Together the data suggest that perturbation of peroxisomal $\beta$-oxidation in oligodendrocytes causes secondary impairment of peroxisomal functions, which precedes and possibly triggers cerebral demyelination. 


\section{Introduction}

\subsection{Peroxisomes}

In an electron microscopic study in 1954 Johannes Rhodin observed small, single membrane bounded structures, which he referred to as microbodies (Rhodin 1954). After the discovery of hydrogen peroxide producing and degrading enzymes in their matrix, microbodies were termed peroxisomes (De Duve \& Baudhuin 1966). Peroxisomes are ubiquitous organelles throughout the eukaryote domain and are found in all nucleated mammalian cells (Lazarow \& Fujiki 1985). Not only the peroxisomal number, but also their size varies considerably between cell types. In the mammalian brain peroxisomes can be as small as $0.1 \mu \mathrm{m}$ in diameter, whereas in liver they can measure up to $1 \mu \mathrm{m}$ (Colton 2004). They are abundant in liver and brain. In the latter they are especially numerous in glial cells, such as oligodendrocytes and astrocytes (TrofferCharlier et al. 1998). The significance of peroxisomes for normal development and maintenance has been shown by the identification of severe diseases related to peroxisomal biogenesis or single enzyme defects.

\subsubsection{Peroxisomal metabolism and its biological importance}

At least 50 proteins with well-established enzymatic activity have been identified in the peroxisomal matrix, of which more than half participate in lipid metabolism. Moreover, enzymes with unknown functions have been described, as well as enzymatic reactions that could not yet be ascribed to specific proteins (Wanders \& Waterham 2006). Apart from luminal enzymes, peroxisomes contain specific integral membrane proteins such as substrate transporters and proteins for peroxisomal matrix protein import (Heiland \& Erdmann 2005).

Peroxisomes play an essential role in a number of catabolic reactions, such as the $\beta$ oxidation of very long-chain fatty acids (VLCFA; > 22 carbon atoms), branched-chain $\mathrm{FA}$, monounsaturated $\mathrm{FA}$, polyunsaturated $\mathrm{FA}$, and their derivatives, the eicosanoides and docosanoids. a-oxidation occurs exclusively in peroxisomes, as well as certain steps of purine-, polyamine-, D-amino acid, ethanol, vitamin $\mathrm{E}$ and $\mathrm{K}$, and cholesterol degradation (Fig. 1; Wanders \& Waterham, 2006). In yeast and plants peroxisomes are the sole location of $\beta$-oxidation, whereas in higher eukaryotes they complement mitochondrial degradation of short to long chain fatty acids, as the sole site for VLCFA degradation. In contrast to mitochondria, peroxisomal $\beta$-oxidation of acyl-CoA to enoyl-CoA by acyl-coenzyme $A$ oxidase (ACOX) results in the formation of $\mathrm{H}_{2} \mathrm{O}_{2}$, which requires detoxification by intraperoxisomal catalase. During mitochondrial acyl-CoA oxidation, $F A D$ is reduced to $F A D H_{2}$ providing protons for the respiratory chain. In peroxisomes enoyl-CoA is further hydrated and subsequently oxidized by the multifunctional Protein 2 (MFP2), also called D-bifunctional Protein (DBP), before a thiolytic cleavage cata- 
lyzed by the acetyl-CoA acyltransferase 1 (ACAA1) releases a shortened FA and an acetyl-CoA. MFP2 also accepts branched chain FA as substrates. FA undergo $\beta$ oxidation until the formation of octanoyl-CoA, which cannot further be degraded in peroxisomes. Resulting acetyl-CoAs and octanoyl-CoAs are carnitylated and exported for degradation and ATP production in mitochondria (Lazarow 1978; Wanders 2014).

Substrates for $\beta$-oxidation are transported across the peroxisomal membrane as activated $C o A$ esters by one of three ATP-binding cassette transporters subtype $D$ (ABCD1; ABCD2; ABCD3; Theodoulou et al. 2006). In addition, diffusion of free fatty acids across membranes has been described (Pillai et al. 2009).

Besides catabolic reactions peroxisomes participate in a number of anabolic reactions including elongation of $F A$, formation of bile acid, and polyunsaturated FA, e.g docosahexaenoic acid (DHA; C24:6), which is an abundant polyunsaturated FA in the brain (Wanders \& Waterham 2006; Jing \& Lim 2012; Itoyama et al. 2012). Furthermore, indications for involvement in cholesterol biosynthesis have been found (Smith \& Aitchison 2013).

In the liver peroxisomes are of vital importance. Apart from participating in the formation of bile acids they are involved in the degradation of various toxins. About $25 \%$ of the ingested ethanol is degraded by liver peroxisomes (Alberts et al. 2002). Moreover, peroxisomes are crucial for proper brain function, especially for myelin, an extremely lipid rich structure that enwraps neuronal processes. Peroxisomal metabolites participate in the formation of several plasma membrane lipid classes. Sphingolipids, form the head group of phospholipids and glycolipids, which are major membrane components. They contain long chain and very long chain FA, mostly C24:0 and C24:1. The latter are exclusive peroxisomal metabolites (Joseph et al. 1972). Plasmalogens form part of the glycerophospholipids, which constitute the biggest fraction of phospholipids in the brain, especially in myelin (Yanagihara \& Cumings 1969; Thomas et al. 1990; Farooqui et al. 2000). The first steps of plasmalogen synthesis are mediated by the peroxisomal enzymes glyceronephosphate O-acyltransferase and alkylglycerone phosphate synthase and their loss of function results in a serve disorder in humans, Chondrodysplasia punctata (Hardeman \& van den Bosch 1991; Hajra 1997). Apart from their importance for membrane dynamics plasmalogens serve as a reservoir for secondary messengers (Brites et al. 2008). Cholesterol, which can be degraded and likely partly be synthesized in peroxisomes, belongs to another main component of plasma membranes, the sterols.

Recently reactive oxygen species (ROS) and reactive nitrogen species (RNS) lost their oath as purely toxic byproducts of cellular metabolism and have become acknowledged as intracellular messengers, if present in subtoxic concentrations (Fransen et al. 2012). They have been implicated in the regulation of cell proliferation, gene expression and programmed cell death (Scherz-Shouval \& Elazar 2011). Furthermore, redox signaling 
can be mediated by ROS and RNS oxidation of cysteine residues, which influences the enzymatic activity of proteins. (Barford 2004). Peroxisomes as platforms of abundant ROS and RNS generation likely play an important role not only in ROS detoxification, but as well in redox signaling. In rat liver $35 \%$ of all $\mathrm{H}_{2} \mathrm{O}_{2}$ generated is derived from peroxisomal oxidases (Boveris et al. 1972). Imbalanced peroxisomal metabolism thus may lead to a failure in redox signaling and to increased oxidative stress as observed in peroxisome related diseases (Galino et al. 2011).

Peroxisomes malfunction has been associated with inflammatory processes by ROS production, but also other peroxisomal products like plasmalogen and DHA serve as precursors for bioactive molecules involved in inflammation, e.g. leukotrienes, prostaglandins, and eicosanoids (Braverman \& Moser 2012). Moreover, eicosanoids are degraded in peroxisomes and play a critical role in inflammatory reactions (Funk 2001).

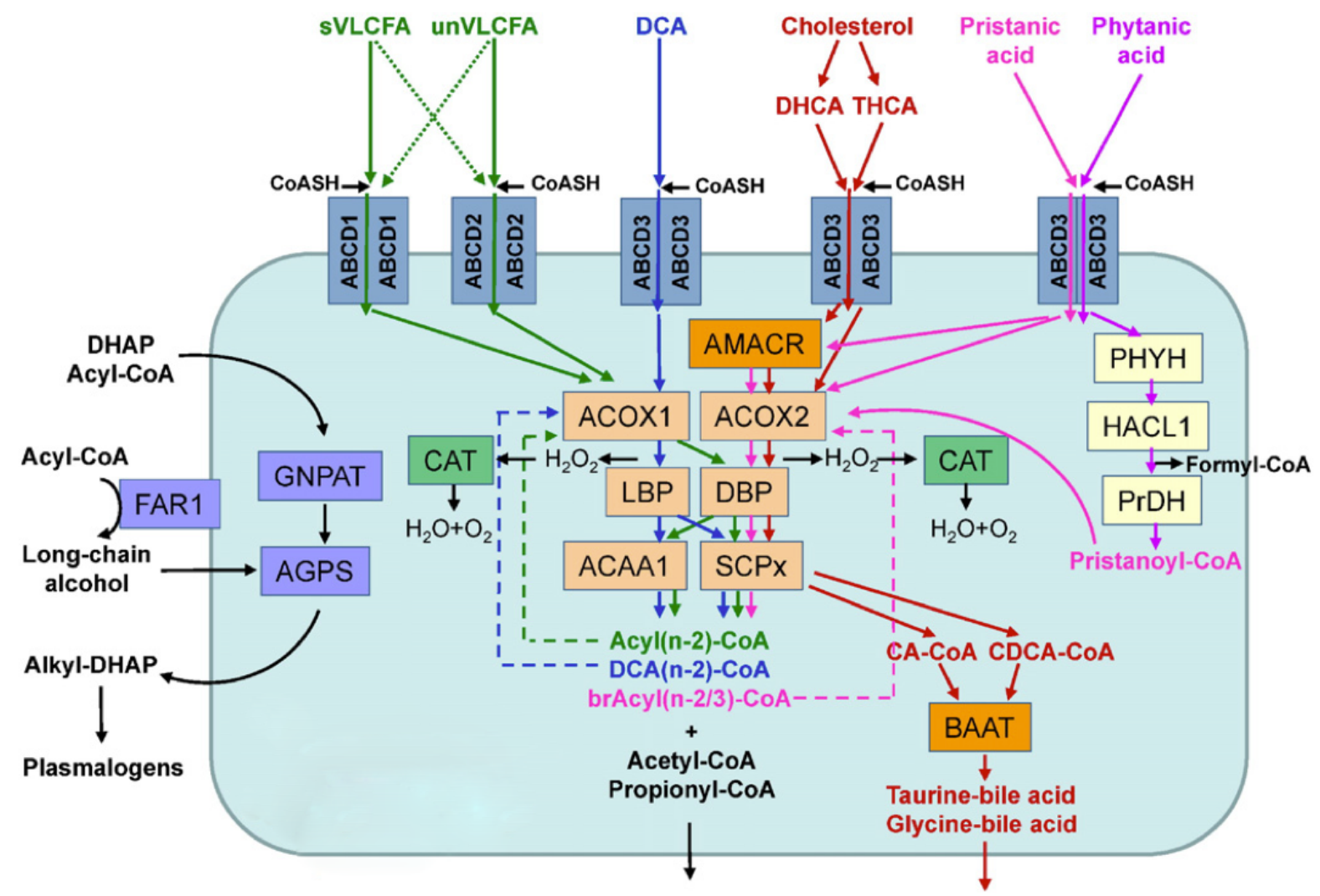

Fig. I: A schematic view of main metabolic pathways in peroxisomes

Enzymes are accentuated by colored boxes according to the different pathways. While enzymes of the Peroxisomal $\beta$-oxidation are highlighted in light orange, $\alpha$-oxidation enzymes are shown in yellow and enzymes involved in bile acid synthesis are depicted in light and dark orange. The different types of substrates for the $\beta$-oxidation are indicated by distinct colors. Abbreviations not mentioned in the text: AGPS = alkyl-dihydroxyacetonephosphate synthase, AMACR $=2$-methylacyl-CoA racemace, $\mathrm{BAAT}=$ bile acidCoA:amino acid N-acyltransferase, brAcyl = branched-acyl, CA = cholic acid, CAT = catalase, CDCA = chenodeoxycholic acid, DCA = dicarboxylic acids, DHAP = dihydroxyacetone phosphate, DHCA = dihydroxycholestanoic acid, FAR1 = fatty acyl reductase 1, HACL1 = 2-hydroxyphytanoyl-CoA lyase, LBP = Lbifunctional protein, $\mathrm{PHYH}=$ phytanoyl-CoA 2-hydoxylase, $\mathrm{PrDH}=$ pristanal dehydrogenase, $\mathrm{SCPx}=$ sterol carrier protein X, THCA = trihydroxycholestanoic acid (Adapted from Waterham et al., 2016) 


\subsubsection{Brain Peroxisomes}

Peroxisomes are involved in the degradation and formation of several metabolites that are highly abundant in the brain. Brain peroxisomes are strikingly smaller than in liver or other tissues. Whereas liver peroxisomes measure between 0.3 and $0.9 \mu \mathrm{m}$ in diameter, peroxisomes in astrocytes and oligodendrocytes have been found to measure about $0.2 \mu \mathrm{m}$ and $0.05-0.1 \mu \mathrm{m}$, respectively and are thus termed microperoxisomes (McKenna et al. 1976; Colton 2004; Kassmann et al. 2011). Peroxisomes are found in all CNS cell types, but interestingly peroxisomes in white matter tracts are frequently associated with the cytoplasmic regions of oligodendrocytes rather than with axons. In neurons peroxisomes are mostly detected in the cell soma (Ahlemeyer et al. 2007; Kassmann et al. 2011). In myelin preparations it was shown that the peroxisomal protein content differs between CNS cell types. Pex5 and Pex14, both peroxins involved in peroxisome protein import, were much more abundant in total brain lysate than in myelin enriched fractions. Contrariwise, Pex11 $\beta$ and catalase were enriched in myelin peroxisomes (Kassmann et al. 2011; and unpublished data). The contribution of peroxisomal metabolism to proper brain function is highlighted by severe CNS pathology seen in peroxisomal disorders patients. Furthermore, impaired myelination and demyelination in Zellweger syndrome spectrum patients emphasize the importance of peroxisomes for myelin.

\subsection{Peroxisomal biogenesis}

Several proteins, so-called peroxins (PEX), are essential for peroxisome assembly and maintenance (Kiel et al. 2006). They enable the targeting and insertion of peroxisomal membrane and matrix proteins, as well as the biogenesis of peroxisomes via de novo synthesis or through growth and division of preexisting peroxisomes. Lack of certain peroxins can either result in the total absence of peroxisomes or in the formation of dysfunctional peroxisomal 'ghosts', if the import of matrix proteins is impaired.

\subsubsection{Matrix protein import}

Different from mitochondria peroxisomes do not contain DNA. Peroxisomal proteins are encoded in the nucleus and synthesized on free ribosomes in the cytoplasm. Two different classes of peroxisomal targeting sequences (PTS) label proteins for peroxisomal import. Most proteins contain the C-terminal target sequence type 1 (PTS1), consisting of the three amino acids Ser-Lys-Leu (SKL) or conserved variants (S/A/C-K/R/H-L; Gould et al. 1989). Different targeting sequences can vary in their efficacy of peroxisomal targeting. A well known example is catalase, which contains a weak peroxisomal targeting signal, the tetrapeptide KANL. PTS1 is recognized by the cytosolic recycling receptor Pex5. The Pex5-cargo complex interacts with the importomer on the peroxi- 
somal membrane consisting of a docking complex and the RING-finger complex. The former is composed of PEX14, PEX13 and PEX17 (Fig. 2; Lazarow \& Fujiki 1985; Léon et al. 2006; Brocard \& Hartig 2014). PEX5 binds to PEX14 and PEX13, which likely results in the formation of an aqueous pore that allows protein translocation of fully folded proteins (Meinecke et al. 2010). After cargo release into the peroxisomal matrix, a process that is not fully understood yet, PEX5 is mono-ubiquitinated by the RINGfinder complex and exported to the cytoplasm via the ATPases PEX1 and PEX6 (Carvalho et al. 2007). In the cytosol the ubiquitin moiety is rapidly removed and PEX5 can enter the next round of protein import (Grou et al. 2008; 2012).

Many proteins carrying PTS1 sequences have been identified so far, but recently additional proteins with PTS1 sequences located downstream of a stop codon were encountered. Only by translational read through of the original stop codon a peroxisomal targeting sequence is appended to these proteins as e.g. in about $1.6 \%$ of transcribed lactate dehydrogenase B (LDHB), which normally localizes to mitochondria (Schueren et al. 2014). Moreover, PEX5 protein import has lately been described to be redox sensitive. Under increased oxidative stress and in aged cells, PEX5 accumulates on the peroxisomal membrane and peroxisomal protein import is decreased (Apanasets et al. 2013).

PTS2 consists of a N-terminally located nonapeptide with the consensus sequence (R/K)-(L/V/I/Q)-XX-(L/V/I/H)-(L/S/G/A)-X-(H/Q)-(L/A) and is recognized by PEX7, which interacts with co-receptors PEX5L, a splice variant of PEX5 (Swinkels et al. 1991; Marzioch et al. 1994). Formerly, it was assumed that PTS2 proteins are imported via the same import machinery as PTS1 proteins. However, Montilla-Martinez and colleagues recently encountered a pore formed by the PTS2 co-receptor Pex18 and the docking complex PEX14 and PEX17 (Montilla-Martinez et al. 2015). A minor portion of proteins without PTS reach the peroxisomal lumen by association to PTS1 or PTS2 containing proteins in a so-called piggy-back mechanism. 


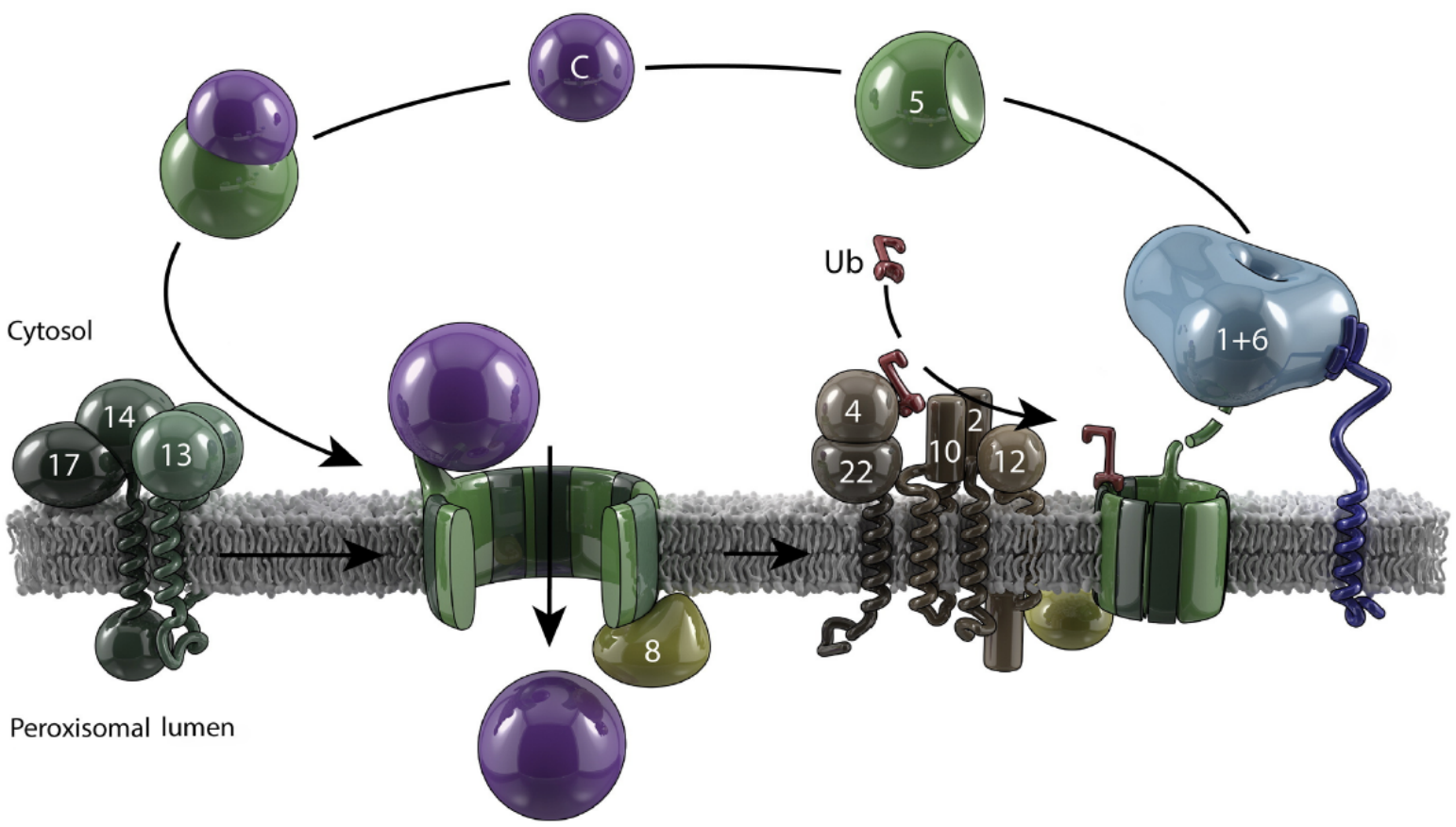

Fig. II: Import of peroxisomal proteins containing a PTS1

The cargo $(C)$ is recognized in the cytosol by PEX5, which subsequently interacts with the docking complex consisting of peroxins 13,14 and 17 to form a transient pore. After cargo release in the peroxisomal lumen PEX5 is ubiquitinated (Ub) by the Ring-finger complex (PEX 2, 10, 12), E2 ubiquitin-conjugating enzyme (PEX4) and the ubiquitin-protein transferase activating protein (PEX22). The receptor release is mediated via the ATPases PEX1 and 6 (Adapted from Meinecke et al., 2016).

\subsubsection{Insertion of peroxisomal membrane proteins}

Protein insertion into the peroxisomal membrane is mediated by PEX3, PEX16, and PEX19. Loss of any of these three peroxins results in the absence of detectable peroxisomal structures (Matsuzono et al. 1999; Fang et al. 2004). PEX19 is a predominantly cytosolic receptor, which recognizes membrane peroxisome targeting sequences (mPTS) and serves as a chaperon for the hydrophilic PMPs in the cytosol (Shibata et al. 2004). PEX3 and PEX16 are integral membrane proteins. A broad range of peroxisomal membrane proteins (PMP) contain MPTSs, which consists of up to two transmembrane segments interspersed with positively charged amino acid residues (Dyer et al. 1996; Brosius et al. 2002). This group of proteins is defined as class I PMPs, in contrary to class II PMPs, which do not interact with PEX19 for peroxisomal targeting (Theodoulou et al. 2006). Class I PMPs bind to the C-terminal region of PEX19 in the cytosol. The receptor-cargo complex subsequently docks to PEX3 in the peroxisomal membrane via the $\mathrm{N}$-terminus. It is speculated that PEX3 perturbs the peroxisomal membrane and thereby facilitates PMP insertion (Pinto et al. 2009). In mammalian cells PEX16, which like PEX3 is a class II PMP, presumably acts as a receptor for both PEX3 and PEX19 (Kim 2006).

Besides the classical class I pathway, class II PMPs have been observed to traffic to peroxisomes via the ER (Geuze et al. 2003). By the insertion of PMPs into the ER spe- 
cialized subdomains can be formed, which are referred to as peroxisomal ER (pER), (Lam et al. 2011; Agrawal et al. 2011). In yeast it has been shown that a part of the machinery involved in the secretory pathway, the Sec61 translocon and the GET machinery, participate in class II PMP insertion into the ER (van der Zand et al. 2010; Thoms et al. 2012). Little is known about the signals necessary for PMP sorting into specialized ER domains. Recently an N-terminal 17 amino acid segment was identified in Pex3p that is necessary and sufficient for intra-ER sorting (Fakieh et al. 2013). Exit of preperoxisomal vesicles is independent of COPII, a protein involved in ER vesicle budding, but it involves PEX3 and PEX19, proteins not related to secretion so far (Sacksteder et al. 2000; South et al. 2000).

In vitro studies using mammalian cells have shown that PEX16 targets to peroxisomes and to the ER where it can mediate insertion of several PMPs (Kim 2006; Toro et al. 2009; Aranovich et al. 2014). Direct insertion of PMP into peroxisomes or indirect trafficking via the ER are not mutually exclusive. Apart from PEX16 also PEX3 and PMP34 have been described to reach peroxisomal localization via both pathways (Aranovich et al. 2014). PEX16 furthermore is required for the exit of PMPs from the ER together with SEC16B, a scaffold protein found in ER exit sites (Kim 2006; Sprangers \& Rabouille 2015).

It is still controversially discussed whether these preperoxisomal vesicles derived from the ER develop into mature peroxisomes or if they serve to replenish preexisting peroxisomes with PMPs and lipids.

\subsubsection{De novo synthesis}

In electron microscopy studies connections between peroxisomes and the endoplasmic reticulum (ER) were observed, providing first evidence for peroxisome de novo biogenesis from the ER (Novikoff et al. 1972). The reappearance of peroxisomes in peroxisome devoid cells upon the reintroduction of the necessary genes demonstrated the possibility of de novo peroxisome formation from the ER in yeast and human fibroblasts (Hoepfner et al. 2005; Toro et al. 2009). However, it is not unambiguously proven yet whether de novo synthesis takes place under normal physiological conditions, or if this is an artifact of peroxisome depleted cells.

The role of the ER for peroxisome biogenesis has been studied in most detail in different yeast strains. Van der Zand et al. presented a detailed model for de novo formation of peroxisomes in S.cerevisiae according to which PMPs are imported into the ER in a Sec61 translocon and a GET complex dependent manner, where they localize to specialized domains (Kim 2006; Schuldiner et al. 2008; Thoms et al. 2012; van der Zand et al. 2012). Interestingly different components of the peroxisomal matrix import machinery localize to distinct ER subdomains. The docking complex (Pex13p, Pex14p), responsible for the first steps of protein import, and a RING finger complex (Pex $2 p$, 
Pex10p and Pex12p) are recruited to different peroxisomal ER (pER) sites (Lam et al. 2011). Subsequently, $p E R$ regions containing PMPs bud off the ER in a Pex19p dependent manner, thereby forming premature peroxisomes with different sets of PMPs and densities (Agrawal et al. 2011). Pex1p and Pex6p mediate a heterotypic fusion of the vesicles, which enables the assembly of functional protein translocon complexes facilitating the import of peroxisomal matrix proteins and finally the formation of mature peroxisomes (Titorenko et al. 2000).

Less details are known about the de novo peroxisome biogenesis in mammals. In contrast to yeast only 13 peroxins necessary for peroxisome formation have been identified in mammals so far (Fujiki 2014). Mutations in three of these peroxins, Pex3, Pex16 and Pex19 result in the absence of any peroxisomal structures (Hua \& Kim 2015). PEX16 has been observed to co-translationally insert into the ER where it can facilitate PEX3 recruitment. PEX3 itself interacts with PEX19 to enable insertion of PMPs (Fang et al. 2004). In two laboratories assays were developed that allow to distinguish peroxisomes generated by growth and fission from peroxisomes formed by de novo synthesis via the presence or absence of fluorescent signals. Both groups observed an increase in the number of fluorescently tagged peroxisome formed by growth and division side by side with the reappearance of unlabeled peroxisomes. These observations though cannot prove the occurrence of de novo synthesis, as unlabeled peroxisomes can also arise by asymmetrical fission or subsequent fission events resulting in a dilution of the fluorescent signal (Kim 2006; Huybrechts et al. 2009).

\subsubsection{Growth and division}

Peroxisomal biogenesis by growth and division occurs via three main events: elongation, constriction, and finally fission. In mammalian cells the integral membrane protein PEX11 $\beta$ is a key player in these processes. PEX11 $\beta$ is a highly conserved protein, which interacts with membrane lipids and initiates the formation of membrane protrusions that elongate into tubular structures. PEX $11 \beta$ is found periodically distributed along elongated peroxisomes and presumably plays a role in membrane constriction (Delille et al. 2010; Koch \& Brocard 2011). Moreover, it is involved in the recruitment of the adaptor proteins mitochondrial fission factor (MFF) and fission factor 1 (FIS1), which interact with the mechanochemical protein, dynamin related protein 1 (DRP1; Itoyama et al. 2013; Koch \& Brocard 2011). Interestingly, mitochondria share all core components of organelle fission like MFF, FIS1, and DRP1, only PEX11 $\beta$ acts exclusively on peroxisomes. DRP1 is mainly found in the cytoplasm and translocates as monomers to membranes, where it polymerases in a GTP hydrolysis dependent manner. Subsequently GTP dependent constriction of the DRP1 ring results in membrane separation and formation of peroxisomes, which rejuvenate by import of newly formed peroxisomal matrix proteins. In mitochondria additional adaptor proteins have been described (MiD49 and 51), each of which can mediate DRP1 recruitment to the mito- 
chondrial membrane (Fujiki 2014). In contrast, in peroxisomes only combined overexpression of MFF and PEX11 $\beta$ results in fission (Itoyama et al. 2013). Recent experiments indicate that PEX11 $\beta$, apart from its dual role in membrane elongation and adapter protein recruitment, plays an additional role in the activation of DLP1 GTP hydrolysis activity driving membrane fission (Williams \& van der Klei 2014).

The existence of both biogenesis pathways is generally accepted, but their relative contribution and regulation remain enigmatic and may vary enormously in different organism and under different physiological conditions (Rachubinski 2014). In mammalian cells the majority of peroxisomes appears to arise via growth and fission under the experimental condition tested (Huybrechts et al. 2009; Delille et al. 2010). It remains to be determined whether ER derived preperoxisomal vesicles mainly deliver PMPs and lipids to preexisting peroxisomes or also contribute significantly to peroxisomal biogenesis by de novo synthesis.

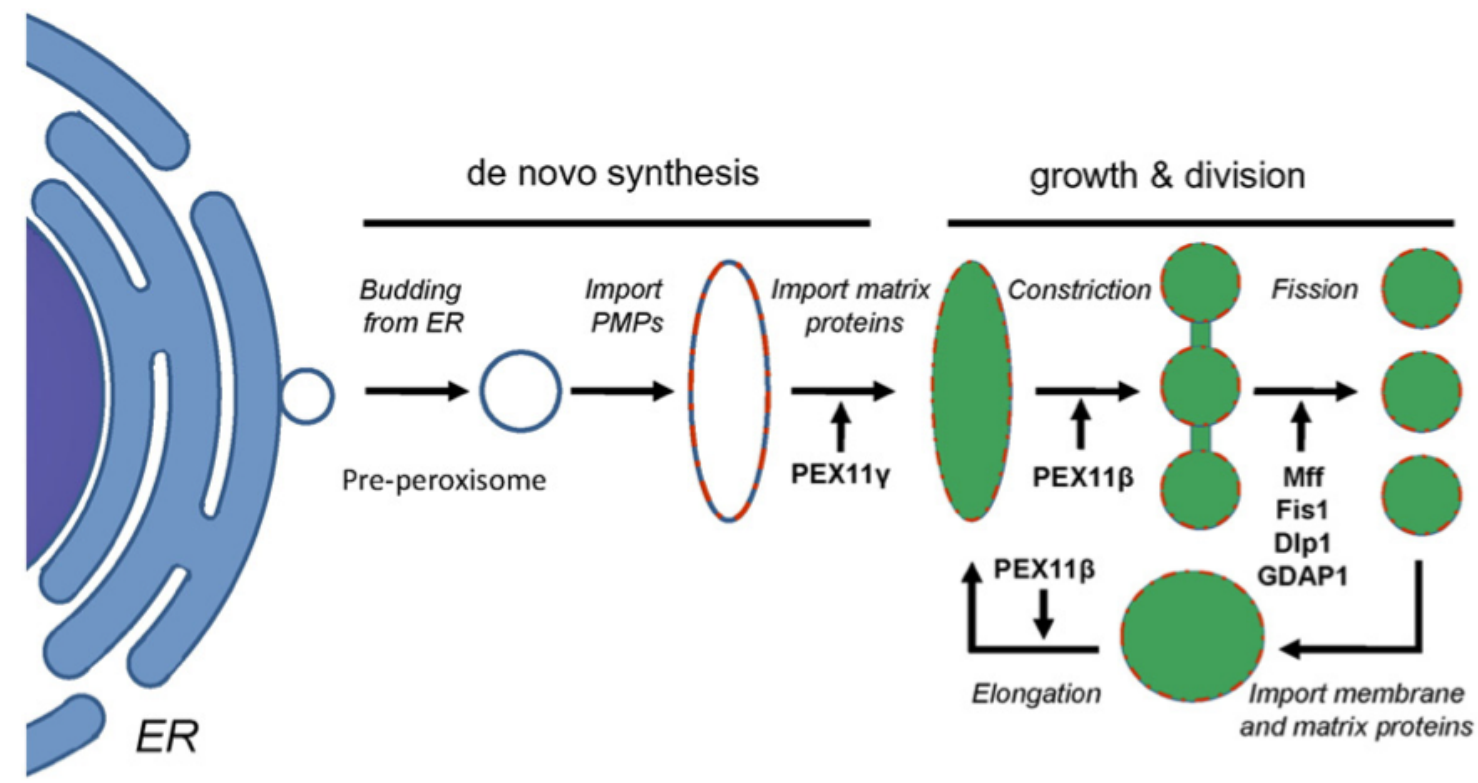

Fig. III: Schematic representation of peroxisome proliferation

It is assumed that peroxisomes can arise via two distinct pathways. (1) De novo synthesis: preperoxisomal vesicles can bud of the ER and develop into mature peroxisomes by the import of matrix proteins and PMPs (left). (2) Growth and division: Mature peroxisomes elongate, constrict and divide resulting in the formation of multiple daughter peroxisomes (right). Proteins known to participate in peroxisomal fission are indicated. (Adapted from Schrader 2016) 


\subsection{Peroxisome dynamics}

\subsubsection{Peroxisome Proliferation}

Peroxisomes are dynamic organelles whose abundance and protein composition is influenced by environmental and developmental signals. First evidence for the influence of exogenous stimuli on peroxisomes was published by Hess et al. in 1965 who observed peroxisome proliferation in rat liver cells in response to treatment with fibrates (Hess et al. 1965). Fibrates and other peroxisome proliferators were later shown to act via members of the steroid hormone receptor superfamily, which were subsequently termed peroxisome proliferator activated receptors (PPAR; Issemann \& Green 1990). Upon ligand binding activated PPARs translocate to the nucleus where they heterodimerize with retinoid-X-receptors and bind to peroxisome proliferator response elements (PPRE) in the DNA to induce gene expression (Kliewer et al. 1992). PPREs consist of tandem repeats of the conserved consensus sequence AGGTCA localized in the promoter region of target genes, such as enzymes of the peroxisomal $\beta$-oxidation (Lemay 2006; Michalik et al. 2006). The three isoforms of PPARs are structurally homologous, but differ in their ligand binding domain and in tissue specific expression patterns (Issemann \& Green 1990; Dreyer et al. 1992).

In the rodent brain all PPAR isoforms are expressed during late embryonic development. Whereas PPAR $\alpha$ and PPARס expression decreases postnatally, PPAR $\beta$ expression remains abundant (O Braissant 2001). Little is known about PPARs in the human CNS, but in situ data indicates that all PPAR isoforms are present in the adult human brain (www.brain-map.org).

Responses to PPARa treatment vary between species. Fibrate treatments in rodents can result in the formation of hepatic tumors, whereas in humans PPARa mediated effects are less pronounced (Peters et al. 2005). In the later PPARa appears to be mainly involved in inflammation, glucose homeostasis, and lipid regulation (Rakhshandehroo et al. 2010). Although PPARa is the most-studied PPAR so far, the mechanism of peroxisome proliferation induction remains elusive. No regulatory effect of PPAR $\alpha$ on Pex11 $\beta$ gene expression, which likely is a major player in peroxisome biogenesis, has been detected (Li et al. 2002).

PPAR $\beta$ and PPARY appear to exert a major role in the brain, as several studies could show beneficial effects of PPAR $\beta$ or PPARY ligand treatment in neurodegenerative diseases (Heneka \& Landreth 2007). Activation of PPAR $\beta$ in oligodendrocyte precursor cells promotes differentiation and myelin maturation (Saluja et al. 2001). The CNS expression of Acyl-CoA synthase 2 (ACCA2), an enzyme of the peroxisomal $\beta$-oxidation, can be regulated by PPAR $\beta$, indicating a role in lipid homeostasis. Moreover, PPAR $\beta$ null mutant mice exhibit altered myelination in the corpus callosum (Peters et al. 2000). PPARY appears to exert a beneficial role in protection against brain inflammation and demyelination as PPARy-deficient heterozygous mutant mice with induced experi- 
mental allergic encephalitis (EAE), a model for multiple sclerosis, show enhanced symptoms (Bright et al. 2003). Furthermore, treatment with the PPARY agonist Pioglitazone improved the outcome of EAE induction in regard to inflammation and demyelination (Feinstein et al. 2002).

Aside from PPARs, members of the PEX11 family seem to be involved in peroxisome proliferation. Mammalians contain three PEX11 variants: PEX11 $\alpha, \beta$, and $\gamma$, each of which is encoded by a single gene. Up-regulation of PEX11 $\beta$ results in peroxisomal elongation and subsequent fission, while depletion leads to increased peroxisomal sizes and strongly reduced peroxisomal numbers (Li \& Gould 2002; Rottensteiner et al. 2003). Pex11 $\alpha$ is known to be induced via PPARa signaling, but has little effect on peroxisome proliferation (McMullen et al. 2014). Light exposure can induce Pex11 $\beta$ expression in plants, but in mammals control of Pex11 $\beta$ expression remains enigmatic (Hu \& Desai 2014). It has been shown that docosahexanoic acid (DHA; C22:6n), a product of peroxisomal $\beta$-oxidation, induces peroxisome proliferation in fibroblast from patients with impaired $\beta$-oxidation, in a PEX11 $\beta$ dependent manner (Itoyama et al. 2012).

\subsubsection{Degradation of peroxisomes}

In mammals three independent systems for degradation of peroxisomes have been identified. 15-Lipoxygenase is a cytosolic enzyme that associates to the peroxisomal membrane and mediates membrane perforation. The peroxisomal matrix is thus exposed to cytosolic proteases, which results in rapid protein degradation (Yokota 2003). LON proteases are serine peptidases, which recognize and degrade misfolded proteins. The peroxisome specific LON protease has been described to degrade proteins in the peroxisomal lumen and might not only be responsible for a reduction of peroxisomal mass, but also for peroxisomal protein quality control (Kikuchi et al. 2004). These two pathways account for about $20-30 \%$ of peroxisomal degradation. The majority of peroxisomes is degraded via autophagic mechanisms, termed pexophagy, which was shown in autophagy impaired Atg $7^{-/-}$mice (Iwata et al. 2006). Selective binding of adapter molecules to the peroxisomal surface and LC3 protein mediate the engulfment of peroxisomes by autophagosomes, which subsequently fuse with lysosomes. Peroxisomal membranes and proteins are subsequently degraded by lysosomal hydrolases (Suzuki \& Ohsumi 2007).

How peroxisomal degradation is regulated in not well known yet. Ubiquitination is a common recognition signal for selective autophagy (Shaid et al. 2012). Ectopic expression of ubiquitinylated PMPs triggered peroxisomal degradation via the adaptor proteins p62 and NBR1 revealing a role for ubiquitin (Ub) also for pexophagy (Kim et al. 2008). However, which proteins serve as physiological targets for ubiquitination and associated regulatory mechanism remain elusive. Recently, two studies revealed a 
ROS induced ATM kinase dependent phosphorylation of PEX5, which lead to monoubiquitination of peroxisome localized PEX5. Ub-PEX5 is subsequently recognized by an adaptor protein, which mediates autophagosome engulfment. ATM kinase is known as a DNA-damage sensor, but was recently found to also reside on the cytoplasmic side of the peroxisomal membrane, a strategic localization for sensing peroxisomal derived ROS. Apart from its role in selective autophagy ATM has been found to enhance the autophagic flux via TSC2 activation, which itself inhibits mTORC1 signaling and thus stimulates autophagy (Nordgren et al. 2015; Zhang et al. 2015). Lately also TSC1 and 2 have been detected on the peroxisomal membrane (Zhang et al. 2013).

Peroxisomal degradation can be induced by treatment and subsequent removal of proliferating agents (Iwata et al. 2006). Furthermore starvation conditions have been shown to result in pexophagy involving PEX14 LC3 interaction (Hara-Kuge \& Fujiki 2008).

\subsection{Central nervous system glial cells}

Glial cells account for about $90 \%$ of all brain cells in humans, whereas neurons make up the residual $10 \%$. Similarly, in rodents the percentage of glial cells $(65 \%)$ outnumbers neurons (35\%; Baumann \& Pham-Dinh 2001). Central nervous system (CNS) glia comprise astrocytes, microglia and oligodendrocytes, which participate i.a. in the trophic support of neurons and regulation of extracellular ion- and transmitter concentrations. Furthermore, they enable enhanced nerve conduction velocity and mediate the immune response.

The name astrocyte derives from the star-shaped morphology of the cells. Astrocytic endfeet interact with endothelial blood capillary cells thereby contributing to the formation of the blood-brain barrier, which tightly controls the entry of substances from the blood (Ballabh et al. 2004). Astrocytes are in close contact to synapses where they not only control the ion homeostasis in the extrasynaptic space, but as well provide metabolic support for neurons and even play a role in synaptic plasticity (Mauch et al. 2001; Pellerin et al. 2007; Suzuki et al. 2012; Saab et al. 2013). Astroglia are activated in response to brain insults like injury, infection and neurodegenerative diseases. Such a reactive gliosis is associated with morphological changes and differential regulation of certain genes e.g. the upregulation of glial fibrillary acidic protein (GFAP; Pekny \& Pekna 2004).

Microglia are the immune cells of the brain. These are monocyte derived cells like their peripheral counterpart, the macrophages. For a long time they were considered as resting cells when not activated, but as the microglial processes were observed to constantly move, it is now believed that in the healthy brain these cells actively and continuously scan the brain parenchyma (Nimmerjahn et al. 2005). When activated they re- 
organize, become motile and migrate towards the site of injury. Furthermore, they can release cytotoxic substances and clear pathogens or apoptotic cells by phagocytosis (Bechmann \& Nitsch 1997; Brown \& Neher 2014). This goes along with a shortening and thickening of the long, branched processed and an increased expression of several genes such as the cytosolic ionized calcium-binding adapter molecule 1 (IBA1) and surface antigens, such as MAC-3 (Ito et al. 1998). Transient microglial activation appears to be beneficial whereas prolonged periods of reactive microgliosis have detrimental effects (Hanisch \& Kettenmann 2007). In the CNS, oligodendrocytes enwrap axons with multiple membranes to form myelin, a lipid rich insulation that enables rapid action potential propagation. Moreover, myelin may provide routes for oligodendrocytes-derived metabolites to trophically support the axonal energy metabolism (Griffiths et al. 1998; Kassmann et al. 2007). Myelination starts prenatally in humans and mice and likely continues throughout life (Young et al. 2014). During embryogenesis oligodendrocyte progenitor cells derive from the neuroepithelium of the ventral neural tube. The majority of these progenitor cells proliferate and mature to myelinating oligodendrocytes (Doetsch et al. 1997). A subpopulation of progenitors, referred to as oligodendrocyte precursor cells (OPC), does not differentiate and remains in a proliferative state. OPCs have a homogeneous distribution throughout the brain parenchyma and are oligodendrocyte lineage restricted. They can develop into mature oligodendrocytes and aid remyelination during brain insults (de Castro 2013).

Oligodendrocytes enwrap up to 20 to 60 individual axon segments with their processes, which broaden at the contact sides and form myelin stacks (internodes) with up to 100 membrane layers. Internodes are regularly interspersed by short unmyelinated regions, the Nodes of Ranvier, which enable electric impulse propagation via voltage-gated sodium channels (Matthews \& Duncan 1971; Simons \& Nave 2016). Myelin is composed of compacted membranes and only the inner and the outer tongue, myelinic channels and paranodal loops, encompassing the Nodes of Ranvier, contain cytoplasm. Compared to other biological membranes myelin contains higher amounts of lipids, which make up 70 to $85 \%$ of the dry mass. They mainly comprise phospholipids (43\%), glycolipids (32\%) and cholesterol (27\%; Evans \& Finean 1965; Stoffel \& Bosio 1997). The lipid rich composition results in a high electrical resistance of myelin, which lowers the axonal capacitance. Myelin lipid composition differs from other membranes. Although there are no unique myelin lipids, cerebosides are much more abundant in myelin than in other biological membranes. Moreover, white matter lipids contain higher levels of saturated long-chain fatty acids (O'Brien \& Sampson 1965). The high percentage of lipids in myelin membranes goes along with a reduced variety of myelin proteins. The proteolipid protein (PLP) and the myelin basic protein (MBP) together make up about $80 \%$ of the protein mass (Boiko \& Winckler 2006). Both proteins are integral membrane proteins. $2^{\prime} 3^{\prime}$-Cyclic nucleotide-3’-phosphodiesterase (CNP) is found in cytoplasmic regions. CNP is an early marker for oligodendrocytes as it is already expressed in early stages of oligodendrocyte differentiation (Scherer et al. 1994). 
For experimental purposes OPCs can be derived from newborn mice and cultured on astrocytes in a fetal bovine containing serum that drives OPC proliferation. OPC are subsequently separated and cultured in a medium allowing OPC differentiation into mature non-myelinating $\mathrm{OL}$ that form myelin-like membrane sheets (McCarthy \& de Vellis 1980).

\subsection{Peroxisomal disorders}

Peroxisomal disorders can be separated into two groups. I) Peroxisomal biogenesis disorders (PBDs), which are caused by mutations in peroxins essential for peroxisome formation and II) single peroxisome transporter or enzyme deficiencies (PTEDs). Xlinked adrenoleukodystrophy (X-ALD), a PTED, shows the highest incidence among peroxisomal diseases, with about 1:17,000 newborn boys affected. Taken together peroxisomal disorders have an expected incidence of about 1:5,000 individuals accounting for a substantial fraction of inborn metabolic diseases (Waterham et al. 2016).

\subsubsection{Peroxisomal biogenesis disorders}

Human peroxisomal biogenesis disorders are autosomal recessive diseases caused by mutations in one of 13 peroxins essential for peroxisome biogenesis and membrane or matrix protein import (Gould \& Valle 2000). These mutations result either in a generalized loss of peroxisomal function (peroxisomal ghosts) or in the case of Pex3, Pex16 and Pex19 mutations in the absence of peroxisomal structures (Matsuzono et al. 1999; Sacksteder et al. 2000). PBDs are subdivided into Zellweger syndrome spectrum (ZSS) disorders and rhizomelic chondrodysplasia punctata type 1 (RCDP). The latter is caused by mutations of Pex7, the cytosolic receptor for peroxisomal proteins, containing a PTS2, which comprise enzymes involved in plasmalogen synthesis and phytanic acid degradation, resulting in deficiency and accumulation of these metabolites respectively (Jansen et al. 2004; Wierzbicki 2007). The clinical phenotype of RCDP1 patients is less severe than in ZSS patients (Rosewich 2005; Trompier et al. 2013).

ZSS disorders are further differentiated into a severe manifestation, the Zellweger syndrome (ZS), an intermediate form, the neonatal adrenoleukodystrophy (NALD) and the least severe presentation, the infantile Refsum disease (IRD). ZSS disorders range from multisystemic disorders resulting in death within the first year of life (ZS) to developmental defects with a higher probability to survive into adolescence or adulthood (NALD and IRD). All encompass malformations of cortex and cerebellum, peripheral neuropathy and inflammatory demyelination (Waterham et al. 2016). 


\subsubsection{Single peroxisome transporter and enzyme deficiencies}

Single peroxisome transporter or enzyme deficiency (PTED) mostly comprise defects in enzymes involved in peroxisomal $\alpha$ - or $\beta$-oxidation or ether lipid synthesis, as well as peroxisomal membrane transporters mediating metabolite transport across the peroxisomal membrane.

Phytanoyl-CoA 2-hydroxylase (PHYH) participates in peroxisomal a-oxidation. Mutations in PHYH result mainly in the accumulation of phytanic acid, which causes a slowly progressive phenotype starting in late childhood, called Refsum disease (Jansen et al. 2004). Apart from $\alpha$ and $\beta$-oxidation peroxisomes play an important role in ether lipid synthesis as they harbor three enzymes necessary for plasmalogen synthesis. Mutations in any of these enzymes result in rhizomelic chondrodysplasia punctata type 2, 3 and 4 (RCDP2-4), which usually exhibit milder pathology than RCDP1 (Ofman et al. 1998; Itzkovitz et al. 2011).

The most common PTED, X-adrenoleukodystrophy, is caused by mutations in the $A B C D 1$ gene, which encodes for a peroxisomal membrane protein. $A B C D 1$ mediates import of straight chain very long chain fatty acids (VLCFA) into peroxisomes, which are further degraded by peroxisomal $\beta$-oxidation (Tanaka et al. 2002; Wiesinger et al. 2013). In patients VLCFA accumulate in cells and plasma. The pathology is extremely variable even within families, but two distinct clinical manifestation can be defined. I) Severe cerebral ALD (CALD) involves cerebral demyelination, neurological deterioration, and premature death. It often already develops in infancy (3-10 years) and is then termed childhood CALD (CCALD). Inflammation is a prominent feature of CALD including reactive gliosis as well as macrophage and lymphocyte infiltration (Moser et al. 1992; Powers et al. 1992; Eichler et al. 2008). II) Adrenomyeloneuropathy (AMN) on the contrary is a slow progressive variant with an onset in the second to third decade of life. It primarily affects the peripheral nervous system and 2/3 of the patients develop adrenocortical insufficiency (Moser et al. 1992). The factors determining the severity of the disease remain elusive.

Patients with peroxisomal $\beta$-oxidation defects are most severely affected among the PTEDs, and show a pathology comparable to ZSS patients. They comprise mutations in Acyl-CoA oxidase 1 (ACOX1), multifunctional protein 2 (MFP2) and acetylCoenzyme $A$ acyltransferase1 (ACAA1). Whereas ACOX1 is solely involved in the $\beta$ oxidation of straight chain VLCFA, MFP2 catalyzes the second and third step in the $\beta$ oxidation of both straight chain VLCFA and branched-chain FA. Accordingly ACOX1deficient patients only exhibit elevated levels of VLCFAs, whereas DBP-deficient patients show increased levels of VLCFA, pristanic acid and bile acid intermediates (PollThe et al. 1988; Ferdinandusse et al. 2005). The clinical presentation of ACOX1-deficient patients is, as observed in X-ALD, very variable. Although the number of described patients is limited, early fatal to mild late-onset phenotypes have been reported (Ferdinandusse et al. 2007). Most MFP2-deficient patients exhibit a severe 
pathology resembling the Zellweger syndrome spectrum, including brain malformation and psychomotor retardation. However, whole exome sequencing identified MFP2-deficient patients with milder presentations surviving into adulthood (Ferdinandusse et al. 2006; Lieber et al. 2014; Lines et al. 2014). Leukodystrophy, the degeneration of brain white matter, is a common pathological hallmark of the ZSS and most diseases caused by dysfunctional enzymes of the peroxisomal $\beta$-oxidation and as well by ABCD1-deficiency.

Peroxisomal $\beta$-oxidation defects and PBDs appear to represent a continuum of phenotypes, which cannot clearly be distinguished by only the biochemical or clinical representation. The historical naming stresses the phenotypic similarities between different peroxisomal diseases. In reference to X-ALD certain clinical phenotypes of the Zellweger syndrome spectrum are referred to as neonatal ALD while ACOX1-deficiency is called pseudoneonatal ALD. The link between biochemical alterations and neurodegeneration in peroxisomal $\beta$-oxidation defects remains unresolved, but the overlapping phenotypes may indicate a common pathomechanism of PBDs and peroxisomal $\beta$-oxidation defects including X-ALD. The severity of the phenotype in peroxisomal $\beta$ oxidation impairment suggests that dysfunction of the peroxisome $\beta$-oxidation is mainly responsible for disease etiology. It could also be envisioned that impaired $\beta$-oxidation might cause the loss of additional peroxisomal functions and explain the similarity between single enzyme defects and peroxisomal biogenesis defects.

\subsubsection{Mouse models of peroxisomal diseases}

The Abcd1 null mouse mutant was the first peroxisomal disease model. It was generated in three independent laboratories in 1997 as a model for X-ALD (Kobayashi 1997; Lu et al. 1997; Forss-Petter et al. 1997). Since then a variety of conventional and conditional mouse mutants have been developed to investigate the pathomechanism of peroxisomal diseases. ABCD1-deficient mice exhibit elevated levels of VLCFA, but fail to develop cerebral neurodegeneration. Instead, a late onset myeloneuropathy is observed similar to AMN patients (Pujol et al. 2002).

PEX5 is the import receptor for most peroxisomal proteins. In its absence peroxisomal structures are formed, which are almost devoid of enzymatic activity (Lazarow \& Fujiki 1985, Yamasaki et al. 1999). Interestingly, $\mathrm{Cnp}^{\mathrm{Cre} / W{ }^{*}} P \operatorname{ex} 5^{-/}$conditional knock-out mice lacking peroxisomal function in oligodendrocytes display inflammatory subcortical demyelination, axonal degeneration and other hallmarks of CALD starting around 4 months of age (Kassmann et al. 2007). These observations suggest that in CALD patients, defects beyond ABCD1-dysfunction might cause demyelination. As demyelinating lesions start in densely myelinated areas of the brain an activity or energy dependent mechanism might be envisioned for the development of secondary peroxisomal defects. 
The ablation of either Acox 1 or Scpx both show rather mild phenotypes in mice and fail to develop cerebral demyelination. MFP2 is a bifunctional enzyme, which catalyzes two central steps in the peroxisomal $\beta$-oxidation. Its absence in man results in a severe ZSS like phenotype. MFP2-deficient mice are indistinguishable from their wildtype littermates at birth but subsequently fail to thrive. One third die within the first three weeks whereas the surviving mice develop progressive motor deficits and die around six month of age. Similar to MFP2-deficiency in humans, mice show elevated levels of VLCFA and impaired bile acid synthesis, but unlike patients they do not exhibit neurodevelopmental defects or cerebral inflammatory demyelination. Instead white matter tracts are spared of reactive gliosis, which is mainly located in grey matter (Baes 2000; Huyghe et al. 2006). To enable the investigation of the consequences of peroxisomal $\beta$-oxidation impairment on demyelination the oligodendrocyte specific

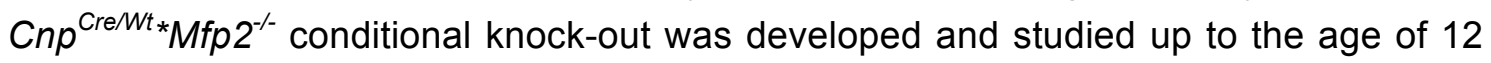
month (Verheijden et al. 2013). Interestingly, mice remained clinically inconspicuous until that age. 


\section{Aims of the study}

Mice lacking peroxisomal function in oligodendrocytes $\left(\mathrm{Cnp} \mathrm{Cre}^{\mathrm{CWt}}{ }^{*} \mathrm{Pex} 5^{-/-}\right)$exhibit reactive gliosis, inflammation, and symmetrical demyelination in the corpus callosum, a major white matter tract of the CNS (Kassmann et al. 2007). This pathology is reminiscent of human peroxisomal diseases that are caused by single mutations in enzymes or transporters involved in peroxisomal $\beta$-oxidation, such as MFP2, ACOX1, and $A B C D 1$. The phenomenon of a similar pathology by naturally occurring peroxisomal enzyme defects on the one side, and by the experimental approach to cell typespecifically ablation of the complete organelle function on the other side, may be explained by a two-step disease mechanism. Impaired peroxisomal $\beta$-oxidation possibly induces a chemical imbalance inside the organelles, especially in cells of metabolically challenged tissue, e.g. in oligodendrocytes of lipid-rich myelin. A perturbed intraorganelle equilibrium may then result in secondary defects and a broad dysfunction of peroxisomes. According to this hypothesis, the rate of peroxisomal turnover may be one important factor to influence such accumulation of defects. A second important factor may be the mode of peroxisomal proliferation, which is in principal possible by both, de novo synthesis from the ER and fission of preexisting peroxisomes.

The aim of this work was to investigate mouse models with impaired peroxisomal $\beta$ oxidation regarding a late-onset cerebral pathology and possible secondary defects in oligodendroglial peroxisomes. To this end first, novel transgenic animals expressing the photoconvertible fluorescent protein mEos2 fused to a peroxisomal targeting sequence (PTS) under control of the oligodendrocyte-specific Cnp-promoter (Cnp-mEos2-PTS1) should be generated. Secondly, a mouse model with cell typespecific ablation of the essential peroxisomal $\beta$-oxidation enzyme, multifunctional protein 2 (MFP2), were to analyze regarding late-onset white matter defects. And thirdly, the conditional MFP2-deficient mice and conventional ABCD1 knockout mice, that only lack peroxisomal $\beta$-oxidation of very long chain fatty acids, shall be intercrossed to CNP-mEos2-PTS mice. These double transgenic animals shall facilitate the analysis of oligodendroglial peroxisomes in mouse models with defects in peroxisomal $\beta$-oxidation in vivo and in vitro. Therefore, an in vitro assay was established that allows to study peroxisomal degradation as well as peroxisomal biogenesis. 


\section{Results}

\subsection{Analysis of aged Cnp ${ }^{\mathrm{Cre} / W t *} M \mathrm{Mfp}^{-/-}$mutant mice}

Mice lacking complete peroxisome function in myelinating glial cells $\left(\mathrm{Cnp}{ }^{\mathrm{Cre} / \mathrm{Wt} *} \mathrm{Pex} 5^{-/-}\right.$ cKO) exhibit symmetrical subcortical demyelination, reactive gliosis and inflammation that is reminiscent of human leukodystrophy patients with a defect only in peroxisomal $\beta$-oxidation. This similarity may be explained by a secondary loss of peroxisome function in patients due to impaired peroxisomal $\beta$-oxidation. To investigate a possible gradual loss of peroxisome function a suitable mouse model was to be developed. The multifunctional protein 2 (MFP2) was a potential candidate as it participates essentially in peroxisomal $\beta$-oxidation. To circumvent developmental effects of MFP2-deficiency and to concentrate on white matter, an oligodendrocyte specific MFP2-conditional mutant mouse $\left(\mathrm{Cnp}^{\mathrm{Cre} / \mathrm{Wt}_{*}} \mathrm{Mfp}^{-/}\right)$was generated. Up to 12 months of age mice were phenotypically inconspicuous, which is not unexpected since diseases caused by peroxisomal enzyme defects often display an age related onset (Verheijden et al. 2013). We investigate the long term effects of impaired peroxisomal $\beta$-oxidation on peroxisome function in metabolically challenged tissue in aged $C n p^{\mathrm{Cre} /{ }^{*}}{ }^{*} M f p 2^{-/-}$mutants $(\geq 16$ months) and focused on the corpus callosum (CC), the brains largest myelinated structure, which is strongly affected in different peroxisomal diseases (Kassmann et al. 2007; van der Knaap et al. 2012).

\subsubsection{Subcortical demyelination}

Progressive symmetrical demyelination of the corpus callosum is a hallmark of several peroxisomal diseases. Mice lacking functional peroxisomes from myelinating oligodendrocytes exhibited a pathology comparable to human patients, as shown by immunohistochemical stainings for myelin proteins and myelin specific dyes (Kassmann et al. 2007). To study white matter integrity in the brain of aged ( $\geq 16$ months) $\mathrm{Cnp}^{\mathrm{Cre} / W{ }^{*}}{ }^{\mathrm{Mfp}} 2^{-}$ /- (hereafter termed mutants) and Mfp $2^{\text {flox/flox }}$ mice (hereafter referred to as CTR) the myelin specific Gallyas impregnation and Luxol fast blue staining for myelin lipoproteins were conducted. Focal demyelination was observed in the Genu, the anterior end of the corpus callosum (Fig. 1A; B), but was less obvious in other myelin structures (not shown). Electron microscopy was conducted in collaboration with Dr. Wiebke Möbius and confirmed the decrease of myelin in the anterior Corpus callosum (Fig. 1C).

Disease progression in patients with peroxisomal $\beta$-oxidation defects is monitored by regular magnetic resonance tomography (MRI) measures. In T2 weighted magnetic resonance images demyelination was revealed by the change from hypointense (dark) to hyperintense (bright) signals in lipid rich tissue. In both patients and conditional $P e x 5^{-/}$mice symmetrical subcortical loss of myelin can be observed by MRI (Kass-

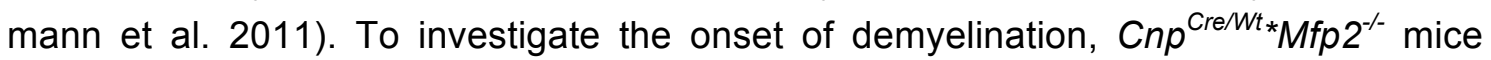


underwent magnetic resonance imaging in collaboration with T. Michaelis (Biomedical NMR, Göttingen) at 2, 6, 14 and 19 months. No alteration in myelin compartments could be observed, indicating a more subtle demyelination compared to patients and conditional $\mathrm{Cnp}^{\mathrm{Cre} / \mathrm{Wt}{ }^{*}} \mathrm{Pex} 5^{-/}$mice (Fig. 1D). Although demyelination was undetectable in MRI the histological findings proved a late onset demyelination in mutants that resembles human peroxisome $\beta$-oxidation defects.

A

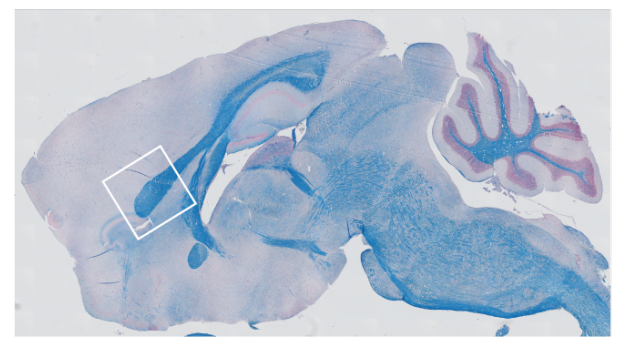

$\mathbf{B}$
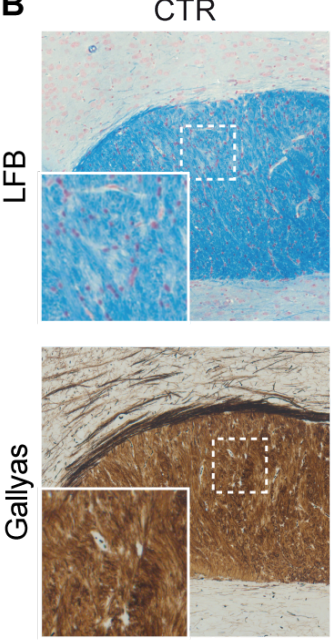

C

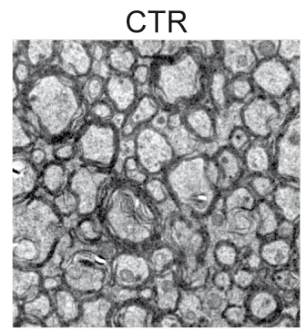

Cnp ${ }^{\mathrm{Cre} W t *} \mathrm{Mfp}^{-/-}$
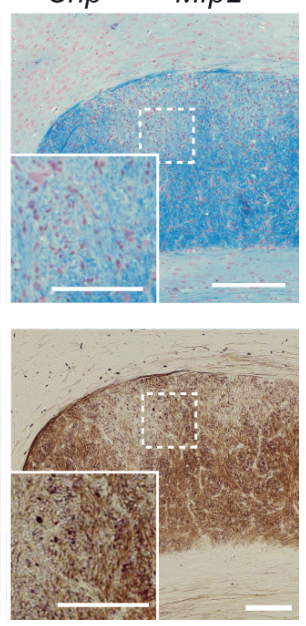

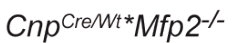

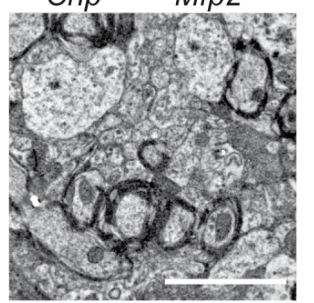

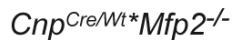

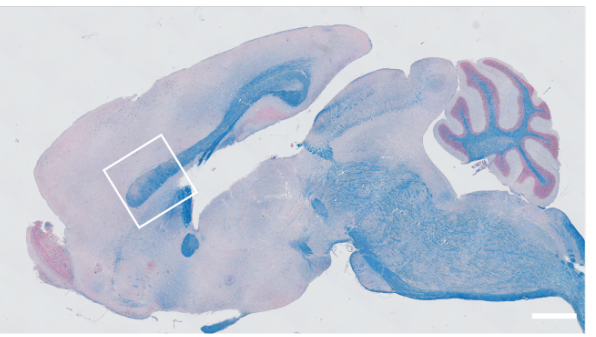

D

CTR

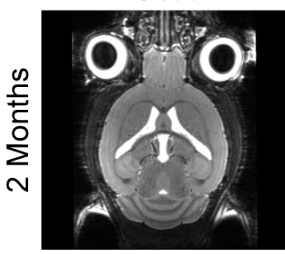

Cnp ${ }^{\mathrm{CreW} t *} \mathrm{Mfp}^{-/-}$
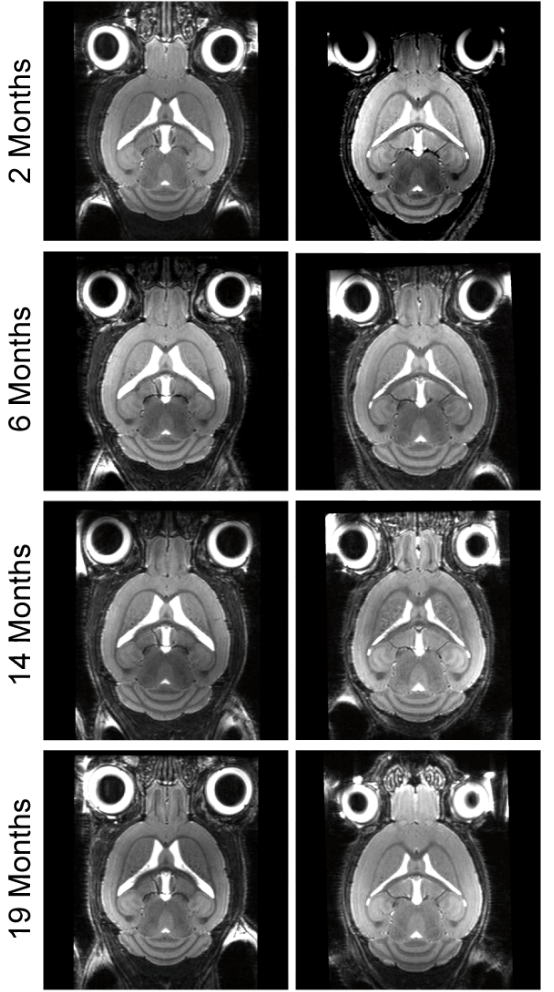

Fig. 1: Subcortical demyelination in $\mathrm{Cnp}^{\mathrm{Cre} / W t_{*}} \mathrm{Mfp}^{-{ }_{-}}$mice

(A) Overview of wax embedded CTR (Mfp2 $\left.2^{\text {flox/flox }}\right)$ and mutant brains; anterior CC highlighted by white box. Scale bar: $1000 \mu \mathrm{m}$. (B) Myelin stainings of sagittal wax sections from $\geq 16$ months-old control and mutant mice. Luxol Fast Blue together with Haematoxilin-Eosin staining (top) and Gallyas silver impregnation (bottom) reveal demyelination of the anterior CC in conditional mutant mice. Images show the Genu of CC and a magnification. Scale bar: $200 \mu \mathrm{m}$; magnified image: $50 \mu \mathrm{m}$. (C) Electron micrographs of the anterior corpus callosum confirm demyelination in $\mathrm{Cnp}^{\mathrm{Cre} / W{ }^{*}}{ }^{M f p} 2^{-j_{-}}$mice. Scale Bar: $500 \mathrm{~nm}$ (D) T2 weighted magnetic resonance images of control and mutant mice at different ages do not show subcortical demyelination. 
Histological alterations were accompanied by altered cage behavior of $\mathrm{Cnp}{ }^{\mathrm{Cre} / \mathrm{Wt} *} \mathrm{Mfp}^{-/-}$ mice, which were readily distinguishable from CTR littermates at $\geq 16$ months. The grid test showed increased numbers of forelimb and hindlimb slips for most mutant animals indicating ataxia (Fig. 2A). Using the elevated beam test, differences were even more prominent. While control mice reached the hiding box failing once at maximum, mutant mice had difficulties to accomplish the task (Fig. 2B). One mutant animal was even excluded from the tests due to complete hindlimb paralysis. Furthermore the mice were scored regarding presence $(+)$ or absence (-) of altered fur appearance, kyphosis, gait abnormalities, tremor, passiveness and hindlimb paralysis. Control mice showed a score distribution between 0 and 2, mutant mice between 3 and 6 . Additionally, bodyweight of mutants was gaining less starting from approximately 11 month of age. Due to mouse-keeping problems the number of animals tested so far is too low to allow statistical analysis. However, the motor impairment of mutants at higher age was apparent and consistent with observations of the same mutants made in another laboratory ( $M$ Baes, personal communication). 
A Grid test

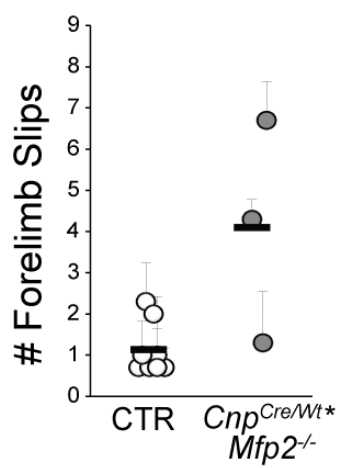

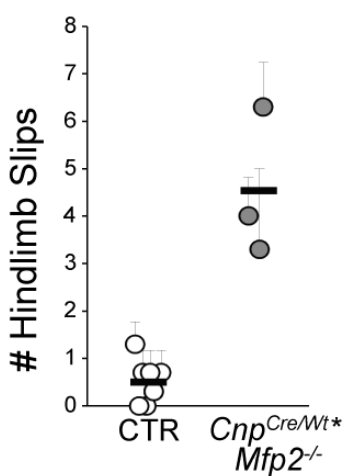

B Elevated beam test

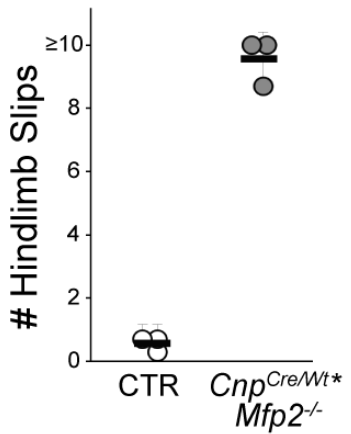

C

\begin{tabular}{|c|c|c|c|c|c|c|c|}
\hline Animal & $\begin{array}{c}\text { Fur } \\
\text { appearance }\end{array}$ & Kyphosis & $\begin{array}{c}\text { Gait } \\
\text { abnormalities }\end{array}$ & Tremor & Passiv & $\begin{array}{l}\text { Hindlimb } \\
\text { Paralysis }\end{array}$ & Score \\
\hline WT 1 & - & - & - & - & - & - & 0 \\
\hline WT 2 & - & - & - & - & - & - & 0 \\
\hline WT 3 & + & - & - & - & - & - & 1 \\
\hline WT 4 & - & + & - & - & - & - & 1 \\
\hline WT 5 & + & - & - & - & - & - & 1 \\
\hline WT 6 & + & - & - & - & - & - & 1 \\
\hline WT 7 & + & + & - & - & - & - & 2 \\
\hline WT 8 & + & + & - & - & - & - & 2 \\
\hline Cnp ${ }^{C r e N t * M f p 2^{-1} 1}$ & + & + & + & - & - & - & 3 \\
\hline Cnp ${ }^{C r e N}{ }^{t *}{ }^{\prime} M f p 2^{-/-} 2$ & + & + & + & + & + & - & 5 \\
\hline Cnp ${ }^{C r e N t *} M_{f p} 2^{-/} 3$ & + & + & + & + & + & + & 6 \\
\hline
\end{tabular}

D

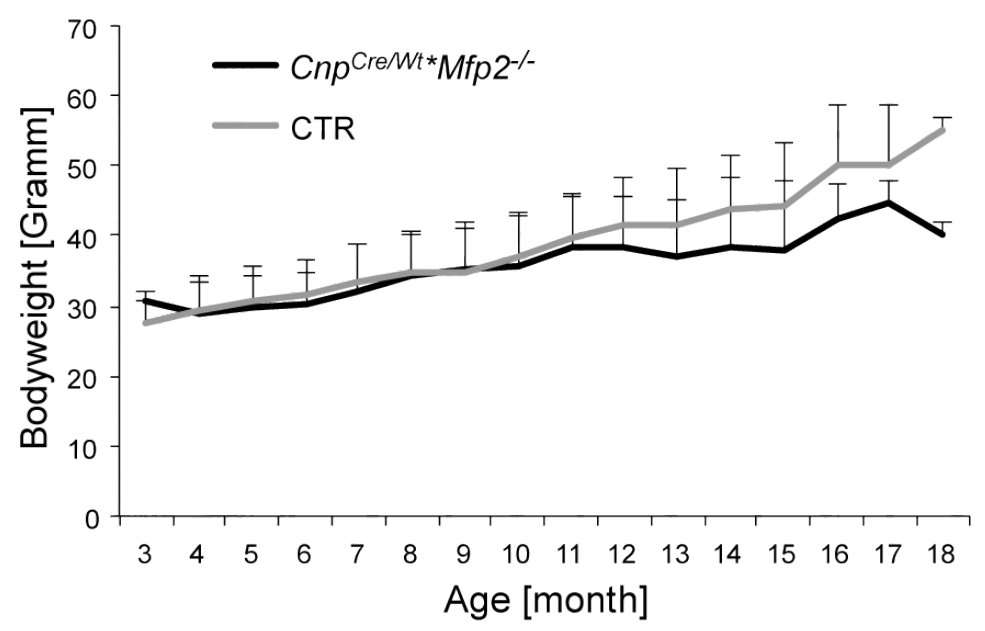

Fig. 2: Clinical symptoms and sings for ataxia in $\geq 16$-month-old $\mathrm{Cnp}^{\mathrm{Cre} / \mathrm{Wt}_{*}} \mathrm{Mfp}_{\mathrm{F}}{ }^{-{ }^{-}}$mutants

(A) The grid test showed increased numbers of forelimb and hindlimb slips for most mutant animals when covering a distance of $80 \mathrm{~cm}$. (B) In the elevated beam test mutant mice had difficulties to accomplish the task, whereas control mice reached the hiding box with no more than one slip. (C) The evaluation of sev-

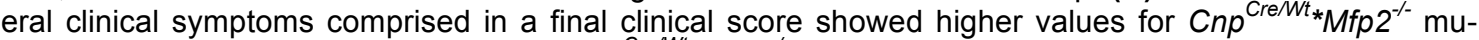

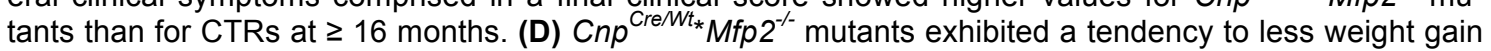
starting at around 12 months. (CTR $n=8$, mutant $n \geq 3$; Mean $+S D$ ). 


\subsubsection{Axonal swellings and neuronal loss}

Myelin is not only important for saltatory impulse propagation, but also for axonal support. Demyelination may therefore result in axonal swellings and neuronal loss (Kassmann et al. 2007). Bielschowsky silver impregnation on sagittal sections of wax embedded brains of $\geq 16$-months-old $\mathrm{Cnp}^{\mathrm{Cre} / \mathrm{Wt} *} \mathrm{Mfp}^{-/-}$and CTR mice allowed the visualization of neurons and axons (Fig. 3A). Similar to demyelination, axonal loss was detectable in the Genu of CC (Fig. 3A).

The amyloid precursor protein ( $\beta$-APP) is transported anterogradely in axons and its accumulation indicates axonal swellings, which precede axonal degeneration. Immunohistological stainings showed $\beta$-APP accumulation in the anterior corpus callosum of all $\geq 16$-month-old $C n p^{C r e / W t *} M f p 2^{-/}$mice analyzed (Fig. 3B). Such APP spheroids were barely detectable in CTR brains. Manual quantification in Fiji showed high variation between animals, which appeared to correlate with disease severity (data not shown).

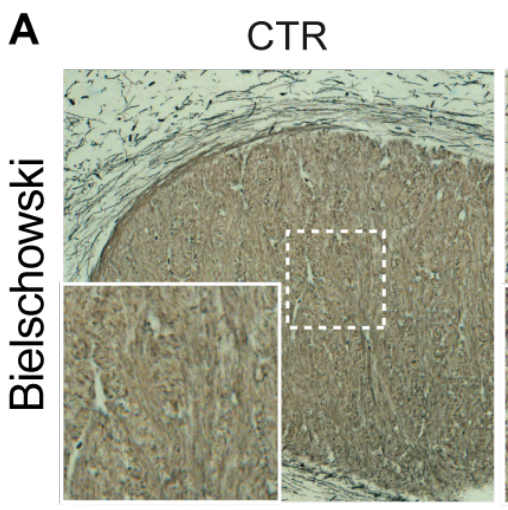

A

CTR

Cnp ${ }^{\mathrm{Cre} / \mathrm{W} *}{ }^{*} \mathrm{Mfp}^{-/-}$

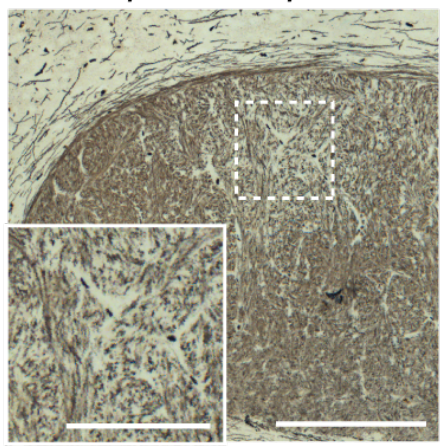

B

CTR

Cnp ${ }^{\mathrm{Cre} / \mathrm{Wt}} \mathrm{Mfp}^{-/-}$
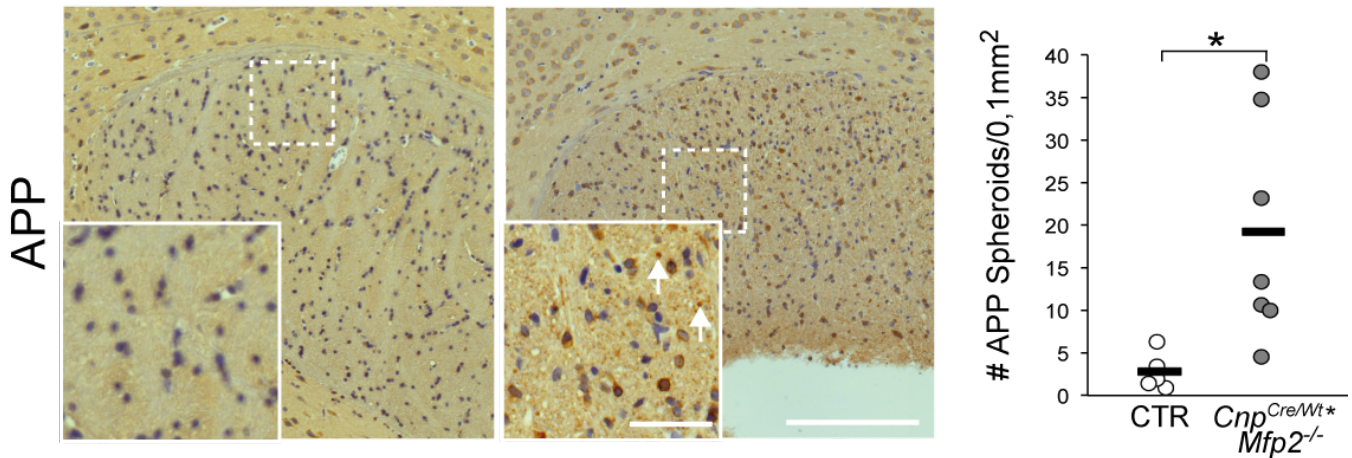

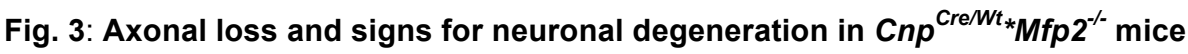

(A) Bielschowsky silver impregnation visualizes neurons and axons on sagittal sections of wax embedded

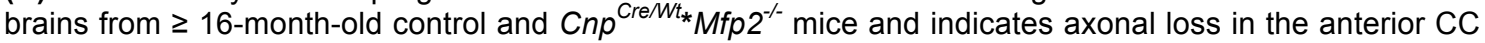
in mutants. (B) $\beta$-APP spheroids (arrows) can be observed by immunohistological stainings on wax embedded brains of $\geq 16$-month-old animals. Quantification reveals a significant increase of $\beta$-APP spheroids in mutant mice (CTR $n=5$, mutant $n=7$; Mean $+S D)$. Scale bar: $200 \mu$ m; magnified image: $50 \mu$ m. 


\subsubsection{Reactive gliosis in the frontal corpus callosum}

CNS damage can elicit responses like proliferation, hypertrophy and altered gene expression patterns in microglia, astrocytes and oligodendrocytes. Together, these processes are referred to as reactive gliosis and can be both beneficial or detrimental for the CNS. During reactive gliosis activated microglia migrate to the site of injury. Their activation results in an increased expression of the cytosolic ionized calcium-binding adapter molecule 1 (IBA1) and of surface antigens such as MAC-3, which are undetectable in quiescent microglia. Astrocytes respond by proliferation accompanied by upregulation of glial fibrillary acidic protein (GFAP). Since reactive gliosis is a feature of peroxisomal defects in humans and in the PEX5-conditional mouse mutant, IBA1, MAC-3, and GFAP immunohistological stainings were carried out. Vibratome sections were investigated for IBA1 signal; paraffin embedded sections were stained for MAC-3. Both proteins indicated strong accumulation of microglial cells in the Genu of corpus callosum of aged ( $\geq 16$ months) mutant mice (Fig. 4 A and B). Immune mediated detection of GFAP on vibratome sections revealed an increase in expression and number of astrocytes (Fig. 4C). Reactive gliosis seemed most prominent in areas affected by demyelination and axonal degradation. 
A
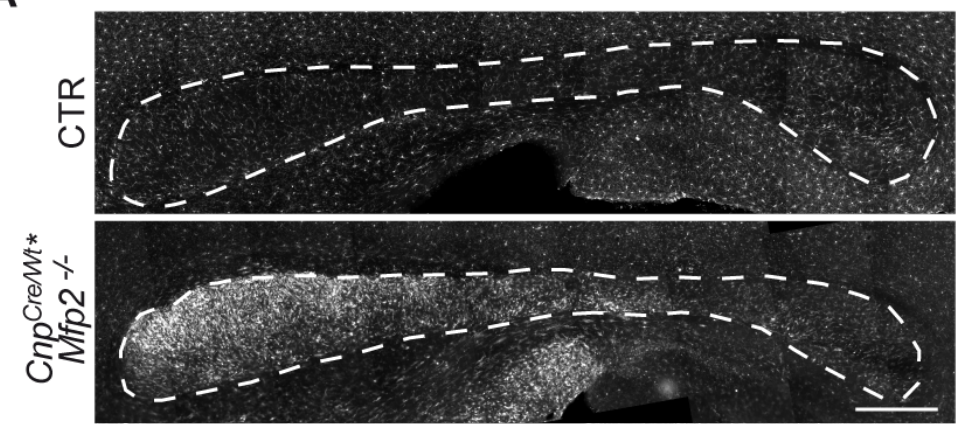

B
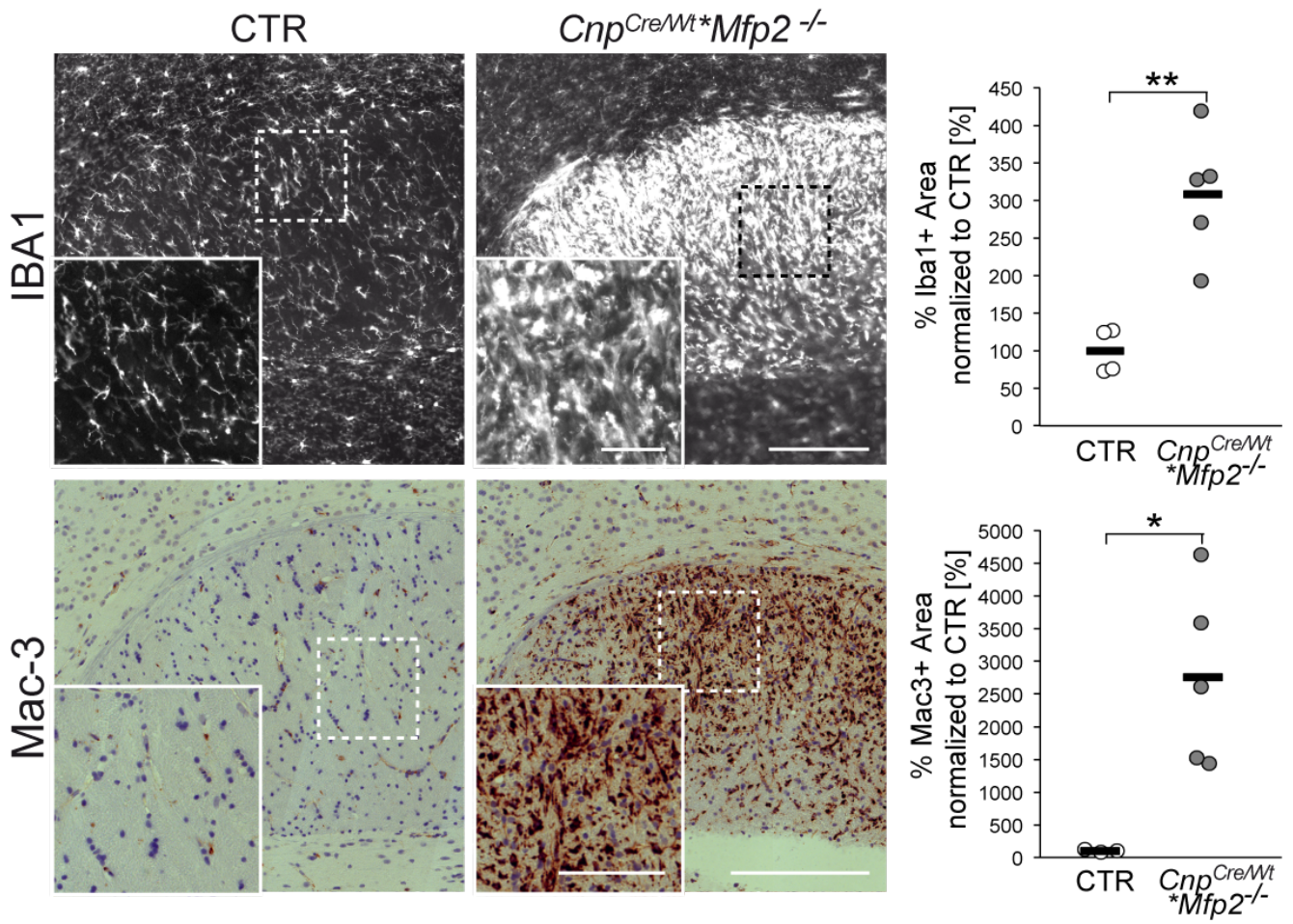

C

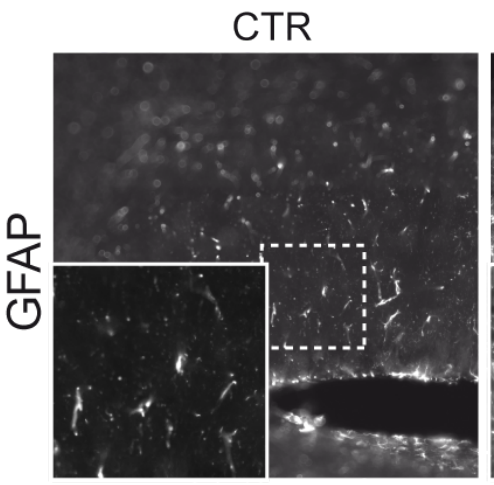

Cnp ${ }^{\mathrm{Cre} N * *} \mathrm{Mfp} 2^{-/-}$
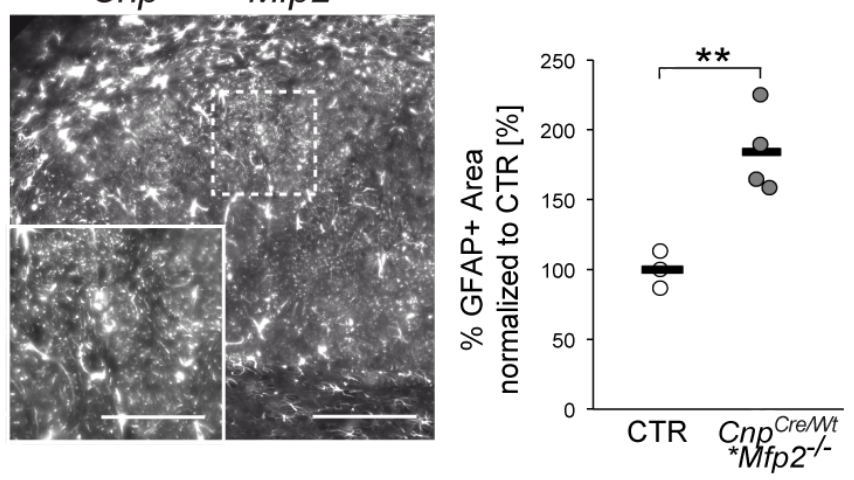

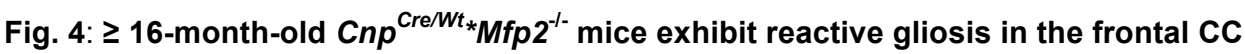

(A) IBA1 antibody staining on vibratome sections visualizes the gradual distribution of microglia along the CC (encompassed by dashed line). Microglial density is highest in the Genu. (B) IBA1 immunohistological staining and quantification prove a significant increase of the IBA1 positive area in mutants compared to CTRs. The microglial surface marker protein MAC-3 is rarely detectable in CTR mice. It is strongly increased in $\mathrm{Cnp}^{\mathrm{Cre} / \mathrm{Wt}_{*}} \mathrm{Mfp} 2^{-/-}$mice, confirming the activated state of microglia. (C) Astrocyte number and GFAP expression are increased in mutants as shown by a immunohistochemical staining against GFAP and quantification of GFAP positive area. (CTR $n \geq 3$, mutant $n \geq 4$; Mean + SD). Scale bar: $200 \mu$ m; magnified image: $100 \mu \mathrm{m}$. 


\subsubsection{T-lymphocyte infiltration in sites of demyelination}

The blood-brain barrier separates the circulating blood from the cerebrospinal fluid impeding the entry of peripheral immune cells. This so-called immune privilege of the brain is affected by certain inflammatory diseases of the central nervous system. In Multiple Sclerosis and in X-linked Adrenoleukodystrophy T-lymphocytes are found in sites of demyelination in the brain. To investigate the presence of T-lymphocytes in our mouse model an antibody staining against the T-lymphocyte co-receptor CD3 was performed on wax embedded tissue of $\geq 16$-month-old mice. CD3 positive cells were pre-

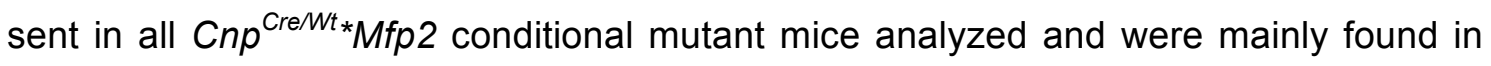
the anterior CC (Fig. 5).
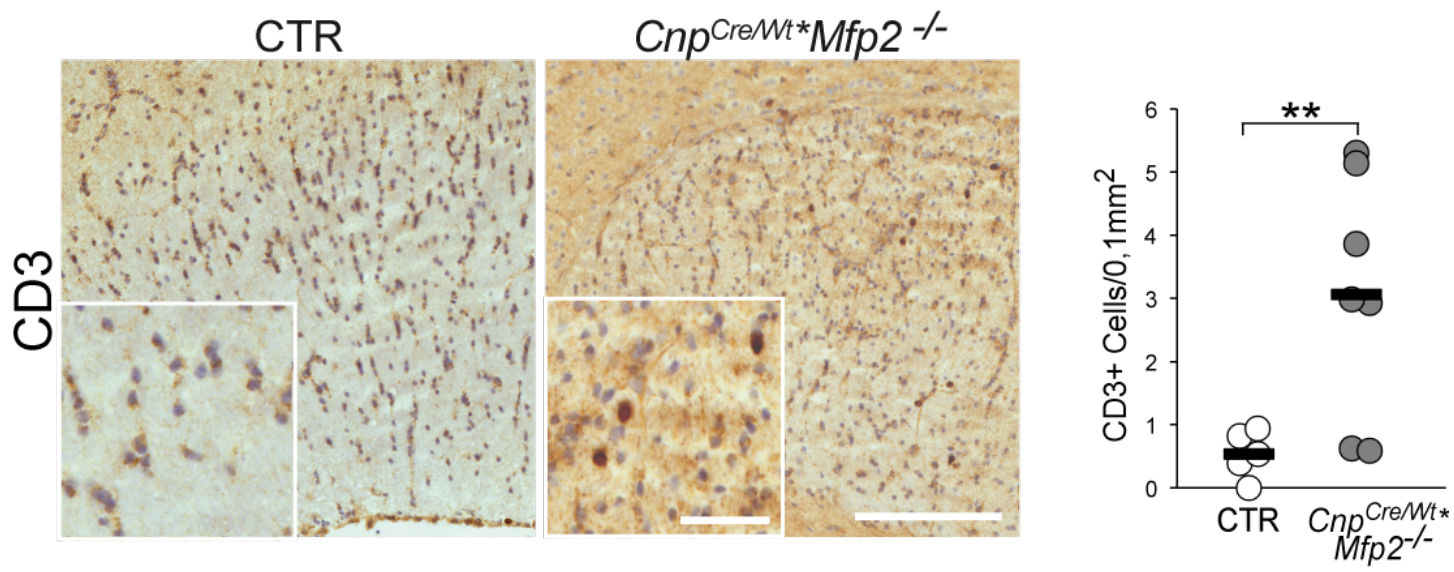

Fig. 5: T-lymphocyte infiltration in the anterior CC in $\mathrm{Cnp}^{\mathrm{Cre} / W t_{\star}} \mathrm{Mfp2}^{-/-}$mice Immunohistochemical detection of CD3 shows an increase of T-lymphocytes in wax embedded samples

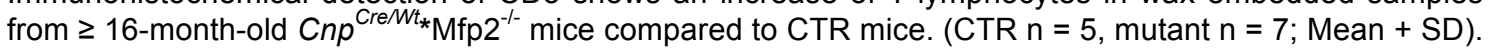
Scale bar: $200 \mu \mathrm{m}$; magnified image: $50 \mu \mathrm{m}$. 


\subsection{Generation and analysis of Cnp-mEos2-SKL transgenic mice}

The high abundance of peroxisomes in CNS cells renders subcellular analysis by traditional immunohistochemical co-labeling techniques difficult. To allow analysis of peroxisomes specifically in myelin-forming glial cells we generated mice expressing a fluorescent protein with a peroxisomal targeting sequence (PTS) under the control oft the oligodendrocyte specific CNP promoter. Employing a photoconvertible fluorescent protein such as mEos2 significantly broadens the spectrum of applications (McKinney et al. 2009). Apart from facilitating the subcellular investigation of peroxisomes it as well enables a pulse-label approach of peroxisomal subpopulations. Data of the following chapter has been published (Richert et al. 2014).

\subsubsection{Generation of Cnp-mEos2-PTS1 transgenic mice}

For the analysis of peroxisomes in myelinating glial cells we chose to express the photoconvertible fluorescent protein mEos2 under control of the myelin specific 2'3'cyclic nucleotide phosphodiesterase (Cnp) promoter. The mEos2 coding sequence was amplified by PCR using an antisense primer, which contained a 9 bp sequence encoding Ser, Lys, Leu, the signal for peroxisomal targeting (PTS1) followed by a stop codon and a restriction site sequence. After restriction digest the $1.1 \mathrm{~kb}$ fragment was ligated into the Cnp1 plasmid, which had been depleted of 57 bp upstream of the start codon responsible for mitochondrial targeting in the CNP2 isoform. Following linearization the fragment flanked by a SV40 PolyA sequence on the 3'end (Fig. 6A) was injected into fertilized C57BL/6N oocytes, from which five potential founders were identified by routine genotyping PCR. The genotyping primers were designed such that a $720 \mathrm{bp}$ fragment was yielded from transgenic, but not CTR animals (Fig. 6B). The offspring of two founders was expanded into separate mouse lines and further analyzed. Mice were born at the expected Mendelian frequency and were indistinguishable from wildtype litter mates.

A
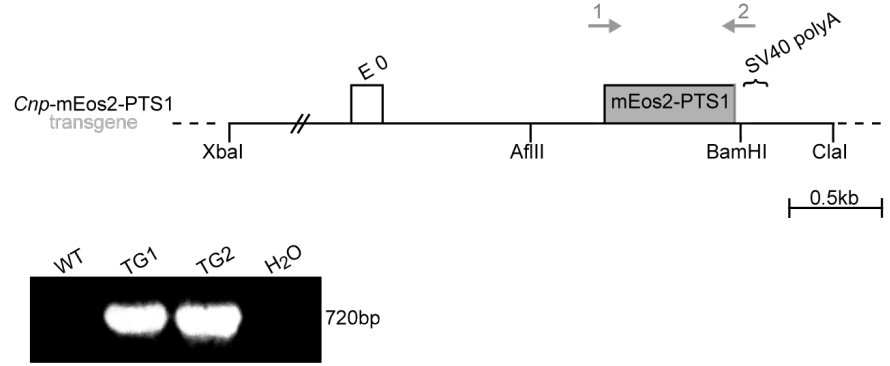

Fig. 6: Structure of the Cnp-mEos2-PTS1 transgene

(A) The $5.1 \mathrm{~kb}$ Cnp-mEos2-PTS1 transgene consists of the Cnp1 promoter encompassing Exon 0 (E 0) followed by the mEos2 coding sequence with a 3 'terminal PTS1 sequence flanked by a SV40 polyadenylation signal. (B) PCR with genotyping primers yields a $720 \mathrm{bp}$ band in the presence of the transgene. No band is detectable in WT. 


\subsubsection{Spatio-temporal expression pattern of Cnp-mEos2-PTS1}

The promoter and the site of integration into the DNA are crucial for transgene expression regarding tissue specificity and expression level. Although the CNP expression pattern is well characterized, the method applied for transgenic mouse generation allowed random integration of exogenous DNA into chromosomes, making further characterization of founder mice necessary.

Of five founders obtained from oocyte injection two were selected and the offspring expanded for further analysis. In this work I will concentrate on the results from one mouse line best suited for peroxisomal analysis in the brain due to relatively even transgene expression throughout the white matter structures of the CNS and appropriate expression levels. Onset and distribution of Cnp-mEos2-PTS1 expression were investigated by wide-field microscopy on vibratome section of transgenic mice at different ages. The punctate pattern of mEos2-PTS1 fluorescence was already detectable in the cerebellum at postnatal day 1 (P1) in line with the developmental expression of CNP starting around embryonic day 13 (Data not shown; Gravel et al. 1998). At P7 fluorescence was observed in the brain stem and spinal cord. Only later also the frontal brain areas were mEos2 positive. At P14 white matter structures such as the corpus callosum, the anterior commissure, and the cerebellar white matter were distinguishable from grey matter by pronounced transgene expression (data not shown). mEos2 expression was visualized in all areas of the CNS with a gradient of stronger expression in the caudal as compared to the rostral region. In mature animals the expression pattern was essentially the same, but fluorescence intensity of the mEos2 signal increased, indicating higher levels of transgene expression (Fig. 7A, B; data not shown). mEos2 expression was absent from most other tissues tested including heart, kidney, liver, lung and muscle. However, Schwann cells, the myelin forming cells in the peripheral nervous system, exhibited extremely low levels of mEos2 signal as seen on teased fiber preparations of sciatic nerves (data not shown), which is in line with published data of low CNP expression in these cells (Sprinkle et al. 1985). Consistent with published data on Cnp-promoter activity mEos2 expression was also found in cells of testis and spleen (Fig. 7C; Weissbarth et al. 1981; Davidoff, Middendorff, Köfüncü, Müller, Ježek, \& Holstein 2002a). 
A
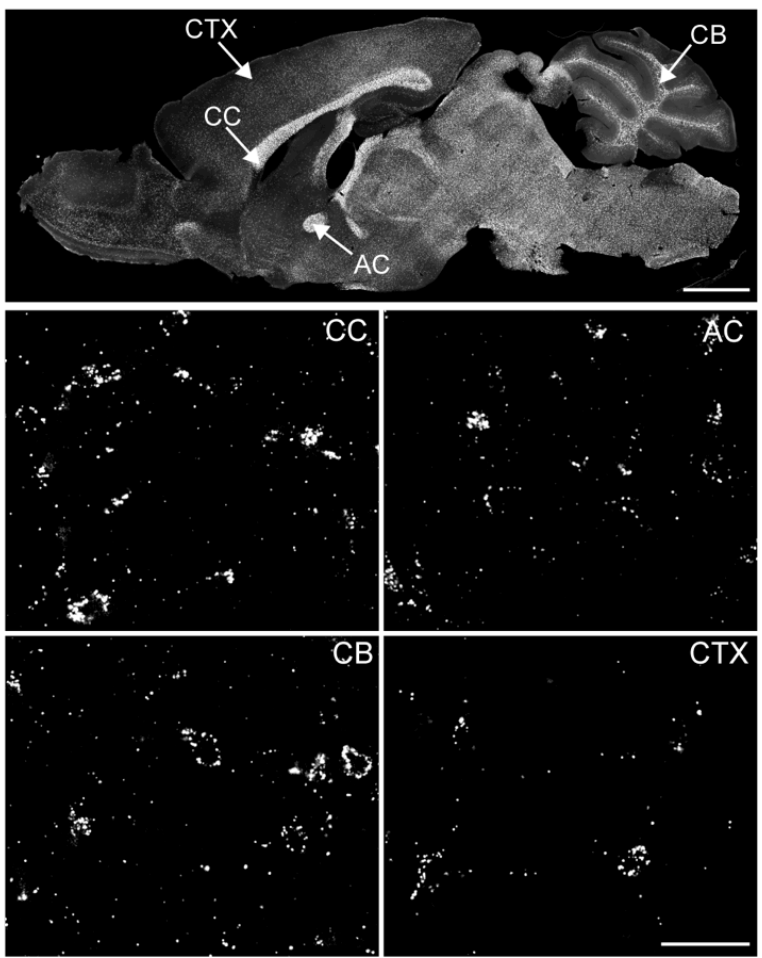

B

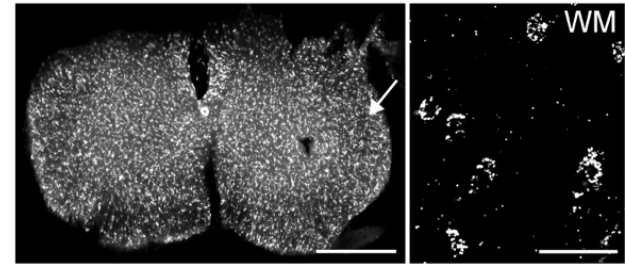

C
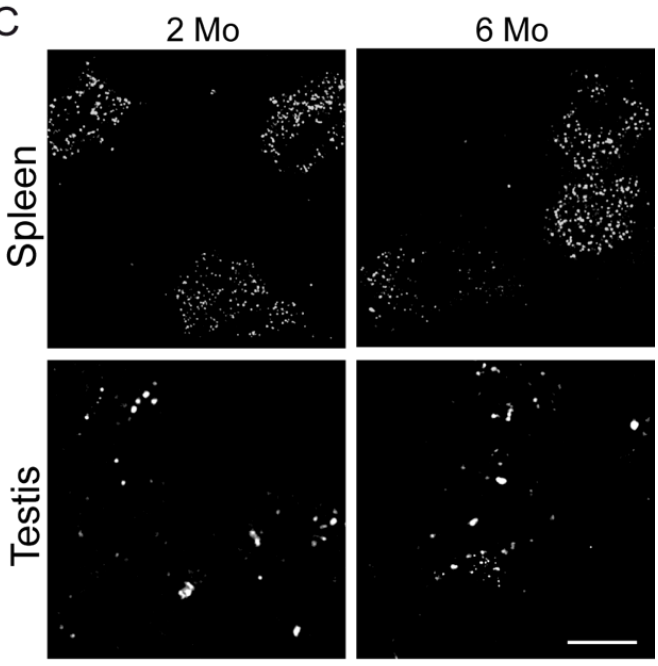

Fig. 7: Expression of mEos2 in the CNS of Cnp-mEos2-PTS1 transgenic mice

(A) Sagittal brain vibratome sections of two-month-old transgenic mice reveal pronounced transgene expression in white matter tracts. Scale bar: $1.5 \mathrm{~mm}$. Middle and bottom panels show magnifications of the corpus callosum (CC), the anterior commissure (AC), the cerebellar white matter $(C B)$ and the cerebral cortex (CTX). Scale bar: $20 \mu \mathrm{m}$. (B) Fluorescence microscopy shows even and abundant distribution of transgene expression in spinal cord cross sections (left; Scale bar $500 \mu \mathrm{m}$ ). Magnification of the spinal cord white matter shows punctate distribution of the fluorescent signal (WM; right; Scale bar: $20 \mu \mathrm{m})$. (C) Punctate pattern of mEos2 fluorescence in spleen and testis of 2 and 6-month-old transgenic mice shows transgene expression in cell types of both organs and indicates peroxisomal localization. Scale bar: $10 \mu \mathrm{m}$

mEos2 showed a punctate subcellular pattern indicating organelle targeting of the transgene. The majority of puncta was accumulated perinuclearly. Cells featuring perinuclear mEos2 signal were identified as oligodendrocytes or oligodendrocyte precursor cells (OPC) by immunohistology stainings on vibratome sections with the oligodendrocyte lineage marker Olig2 (Fig. 8A). Single mEos2 puncta not associated with nuclei were expected to reside in cellular processes and cytoplasmic compartments of myelin such as the innermost and outermost myelin wrap, as well as in paranodal loops.

Quantification revealed that the majority of Olig2 positive nuclei were associated with a perinuclear mEos 2 signal. $60-80 \%$ of the oligodendrocyte lineage cells in the corpus callosum, the cerebellar white matter, the anterior commissure and the cortex showed colocalization with mEos2 puncta (Fig. 8B). 

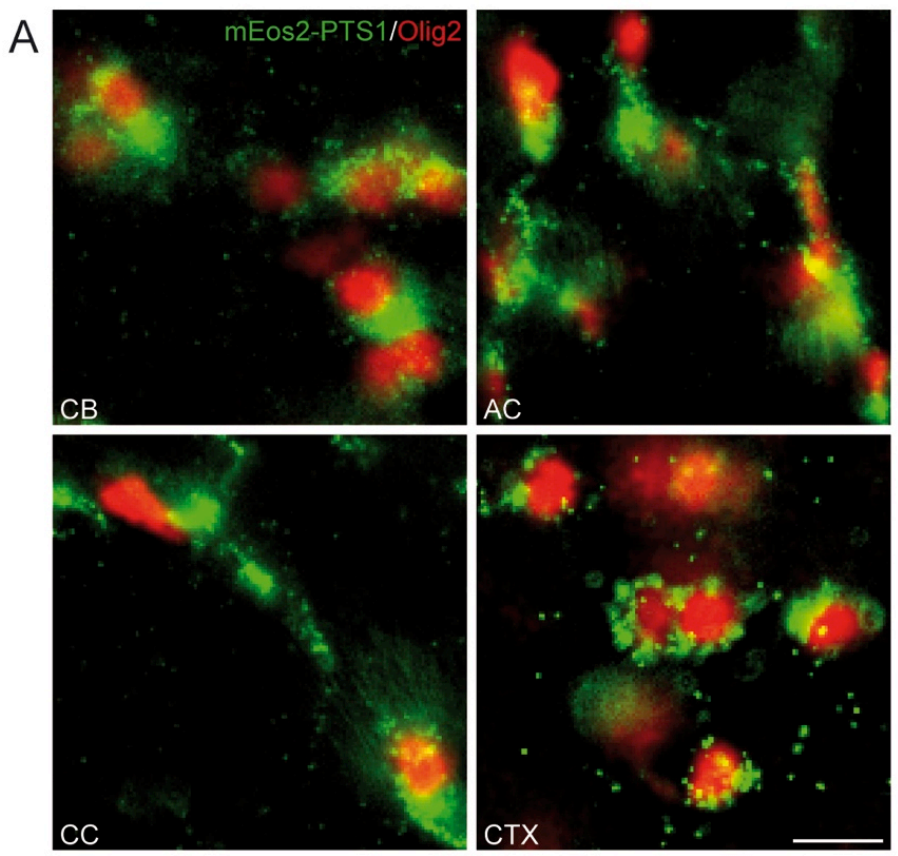

B

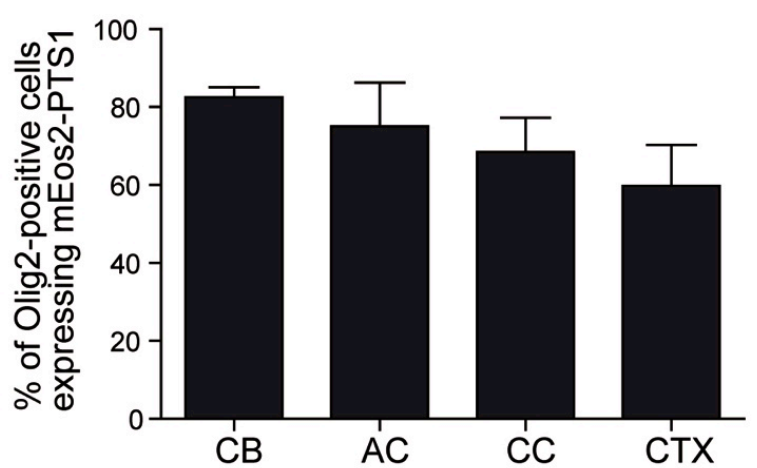

Fig. 8: The majority of Olig2+ cells in Cnp-mEos2-PTS1 transgenic mice show perinuclear mEos2+ puncta

(A) Immunostaining for the nuclear oligodendrocyte line-age marker Olig2 (red) on Vibratome sections shows frequent association of oligodendroglial lineage cells with perinuclear mEos2+ puncta. (B) Quantification of Olig2+ nuclei with perinuclear mEos2 signal in the cerebellar white matter (CB) anterior commissure $(A C)$ corpus callosum (CC) and in the cortex (CTX) reveals $\geq 60 \%$ of transgene expressing oligodendrocyte lineage cells. (Mean $+\mathrm{SD} ; \mathrm{n}=3$ ) Scale bar: $50 \mu \mathrm{m}$

To exclude transgene expression in other cell types of the CNS immunostainings on vibratome sections were performed with antibodies against marker proteins for neurons $(\mathrm{NeuN})$, astrocytes (GFAP) and microglia (IBA1; Fig. 9). Perinuclear distribution of mEos+ vesicles was never found in close association with any of these marker proteins, indicating exclusive transgene expression in oligodendrocytes and probably oligodendrocyte precursor cells in the brain. 


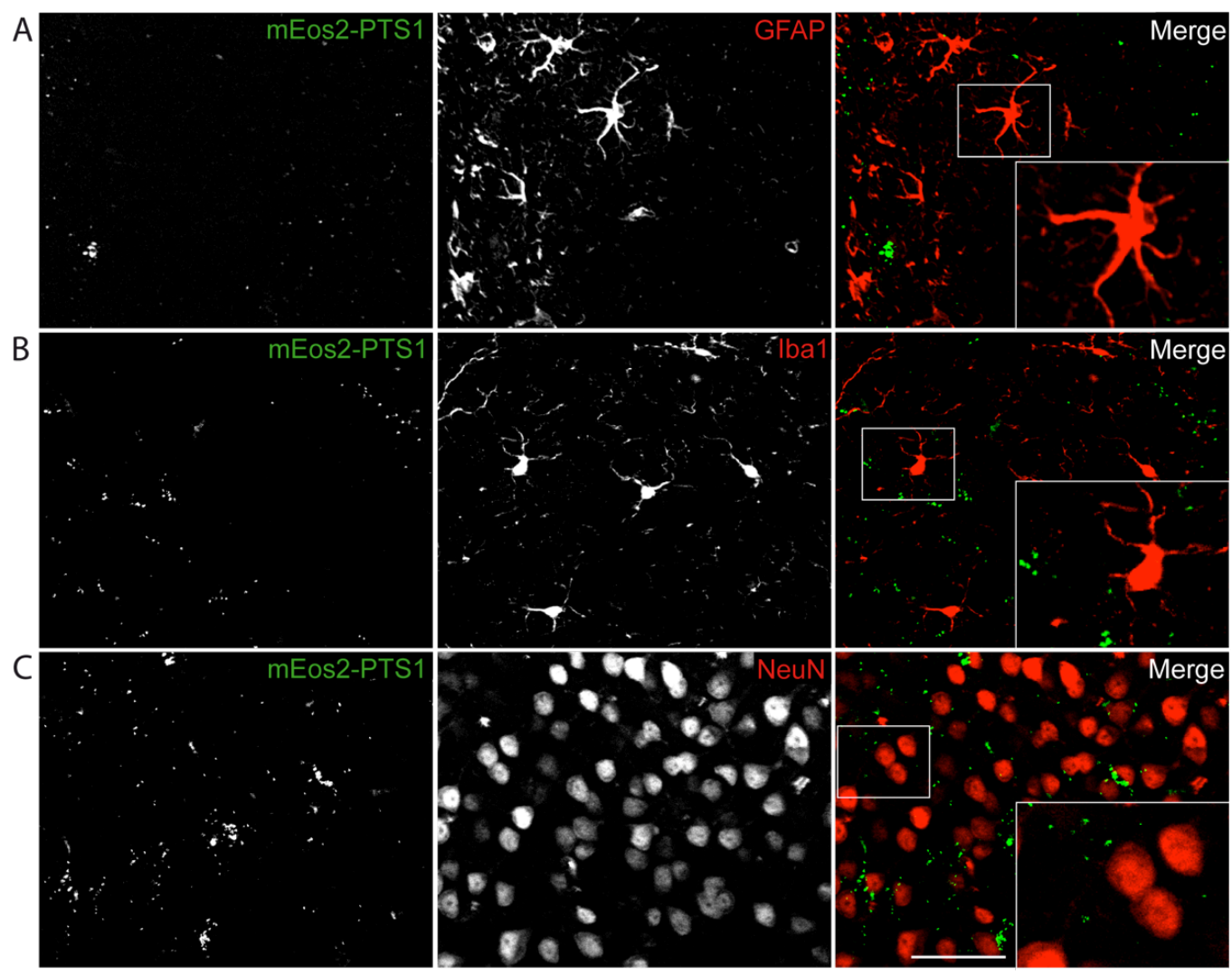

Fig. 9: mEos2+ puncta are absent from astrocytes, microglia, and neurons Immunohistochemical labeling on vibratome sections from 2-month-old Cnp-mEos2-PTS1 transgenic mice for (A) Astrocytes (GFAP; red) (B) Microglia (IBA1; red) and (C) Neurons (NeuN; red) show the absence of perinuclear mEos2+ puncta (green) from cells other than oligodendrocyte lineage cells. Scale bar: $50 \mu \mathrm{m}$

\subsubsection{Peroxisomal targeting of mEos2-SKL}

The C-terminal tri-peptide Ser, Lys, Leu serves as a peroxisomal targeting sequence (PTS), which is recognized and shuttled into the peroxisome by the soluble recycling receptor PEX5. Punctate distribution of mEos2 fluorescence indicates vesicular distribution of transgenic mEos2 protein. To confirm the peroxisomal localization two peroxisomal marker proteins were chosen for immunohistochemical co-labeling on vibratome sections of 2-month-old Cnp-mEos2-PTS1 transgenic animals. Acetyl-CoA acyltransferase (ACAA1; Fig. 10A) is an intraperoxisomal enzyme involved in peroxisomal $\beta$ oxidation. The peroxisomal membrane protein 70 (PMP70; Fig. 10B) is an ABCtransporter, an integral membrane protein of peroxisomes. Both marker proteins (Fig. 10, red) showed substantial colocalization with the mEos2 signal (Fig. 10, green). Peroxisomes that were positive for peroxisomal markers, but not for mEos2 are likely to reside in other cell types than oligodendrocyte lineage cells. To exclude targeting of the transgene to other organelles than peroxisomes, mitochondria were visualized by staining for mitochondrial ribosomal protein S21 (MRP-S21; Fig. 10A) and lysosomes by the detection of lysosome-associated membrane protein 1 (Lamp1; Fig. 10D). mEos2 signal was never found to colocalize with any of these markers proteins. 

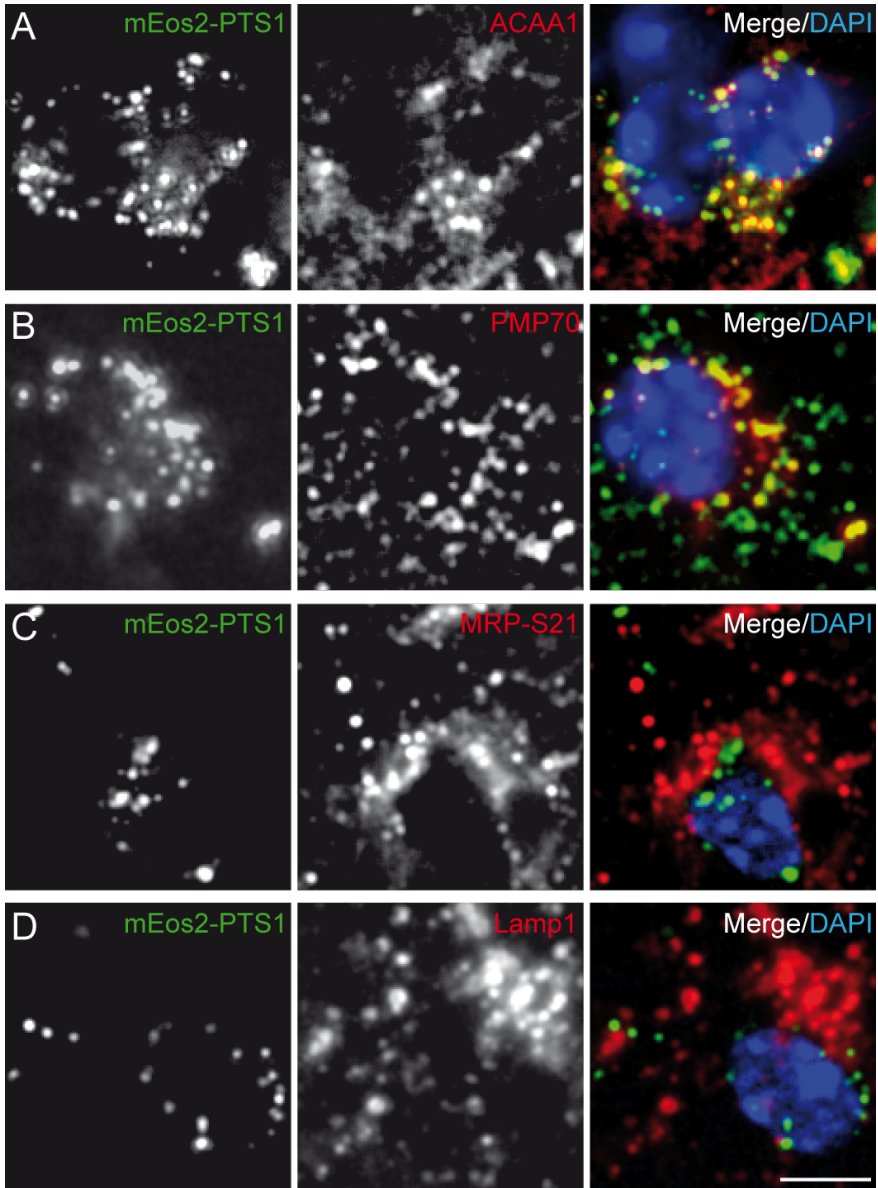

Fig. 10: mEos2 targets exclusively to peroxisomes in Cnp-mEos2-PTS1 mice

Immunohistochemical co-labeling on vibratome sections with the intraperoxisomal marker proteins (A) Acetyl-CoA acyltransferase (ACAA1; red) and (B) the peroxisomal membrane protein 70 (PMP70; red) show peroxisomal location of the mEos2 signal (green). Co-staining for (C) the mitochondrial ribosomal protein S21 (MRP-S21; red) and (D) the lysosome-associated membrane protein 1 (Lamp1; red) did not show colocalization, excluding mistargeting of the mEos2 protein to these organelles. DAPI-stained nuclei are shown in blue. Scale bar: $5 \mu \mathrm{m}$.

\subsubsection{Photoconversion of mEos2}

The $\mathrm{pH}$ may have an impact on fluorescent proteins (FP). Peroxisomal $\mathrm{pH}$ has been shown to differ from the cytosol and may thus result in altered mEos2 performance (Dansen et al. 2000; Jankowski et al. 2001). To optimize mEos2 visualization the actual excitation and emission spectra of intraperoxisomal mEos2 in live Oli-neu cells, an oligodendroglial cell line, was determined. Cells were transfected with the same construct used for transgenic mouse generation. Two days after transfection a lambda scan was conducted on live cells incubated at $37^{\circ} \mathrm{C}$ and $5 \% \mathrm{CO}_{2}$ concentration with excitations between $470 \mathrm{~nm}$ and $670 \mathrm{~nm}$ and a $10 \mathrm{~nm}$ step-width. The 2D contour plot shows maximum emission at $530 \mathrm{~nm}$ when excited at $509 \mathrm{~nm}$ (Fig. 11). This is comparable to the published spectral properties with a maximum emission at $519 \mathrm{~nm}$ upon excitation at $506 \mathrm{~nm}$ (McKinney et al. 2009). 


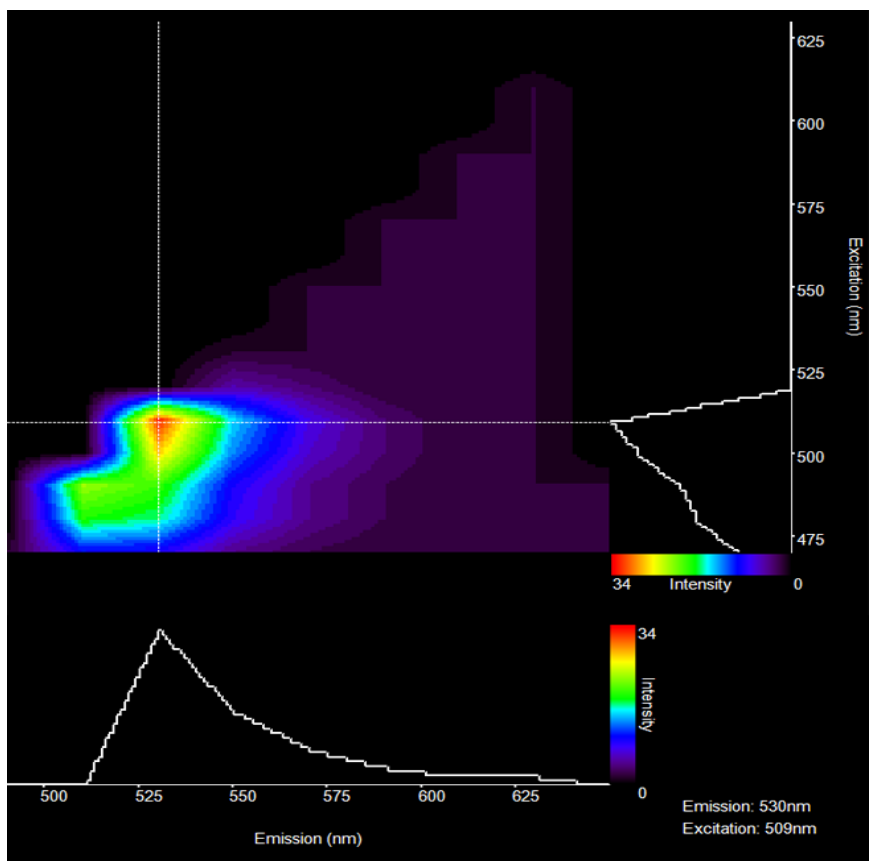

Fig. 11: 2D contour plot from a $\lambda$ scan of Cnp-mEos2-PTS1 transfected cells confirms chromophore functionality

$X$-axis shows emission peak at $530 \mathrm{~nm}$ upon excitation at $509 \mathrm{~nm}$ (y-axis), which is comparable to the published spectral properties.

Photoconvertible FPs enable tracing of subpopulations by pulse-chase experiments. The mEos 2 protein is photoconverted by $\approx 370 \mathrm{~nm}$ light irradiation, which catalyzes the cleavage of an amide bond between two amino acids in the chromophore resulting in a red shift of fluorescence emission. A protocol for photoconversion of the mEos2 protein in live cells was adapted on an inverted microscope equipped for live-cell imaging with live Oli-neu cells expressing the Cnp-mEos2-PTS1 construct or fibroblasts expressing mEos2-PTS1. Conversion from green to red was achieved by irradiation with a Dapi Filter cube (340 nm to $390 \mathrm{~nm}$ ) at high output intensity levels of the mercury short arc illumination source. Following $\approx 370 \mathrm{~nm}$ light exposure single red peroxisomes were readily detectable, but absent from regions not exposed to $\approx 370 \mathrm{~nm}$ light (Fig. 12A). The protocol was designed such that only a portion of the intraperoxisomal mEos2 protein was switched to red fluorescence. Conversion of the total mEos2 pool resulted in pronounced bleaching and phototoxicity. Using a 63x objective 1,200 ms excitation resulted in roughly the same emission intensity of red and green. The employment of an adjustable rectangular diaphragm allowed photoconversion of a discrete number of peroxisomes while the majority remained unswichted as shown using fibroblasts (Fig. 12B). 
A
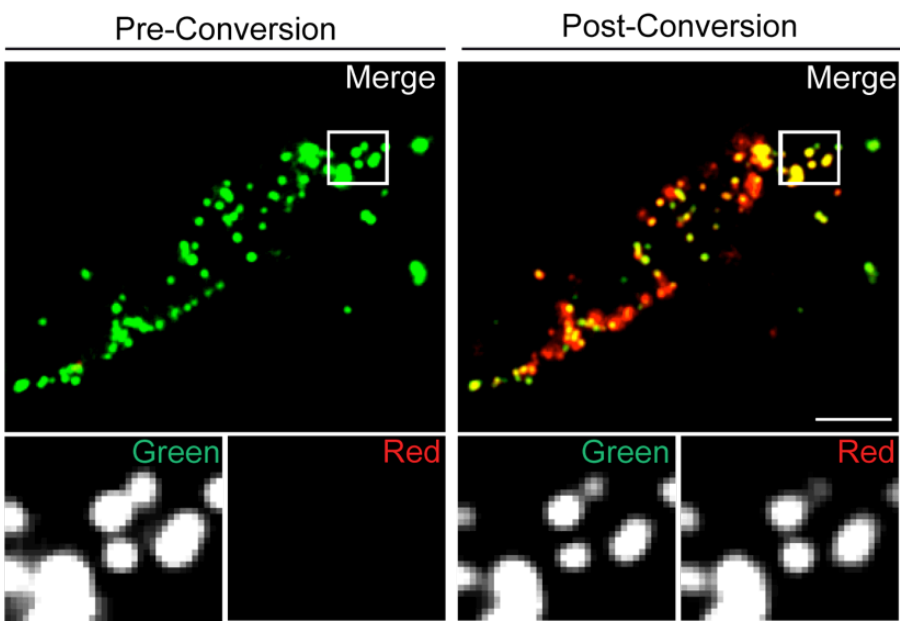

B

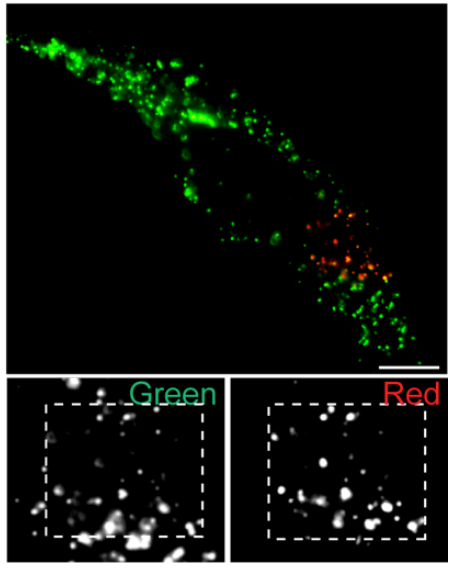

Fig. 12: Photoconversion of live cells expressing mEos2-PTS1

(A) Cultured Oli-neu cells transfected with Cnp-mEos2-PTS1 exhibit strong green, but no red fluorescence. Upon irradiation with $\approx 370 \mathrm{~nm}$ light punctate red fluorescence is detectable, which colocalizes with green mEos2+ puncta. Scale bar: $5 \mu \mathrm{m}$ (B) A subpopulation of peroxisomes can be photoconverted by using a rectangular diaphragm (indicated by dashed line in magnification) in live fibroblasts expressing mEos2-PTS1. Scale bar: $10 \mu \mathrm{m}$

The same photoconversion protocol was applicable to PFA fixed tissue (Fig. 13). With lower magnification objectives $\approx 370 \mathrm{~nm}$ light exposure time had to be increased. Conversion was achieved in different brain areas of fixed tissue such as $C C$ and $C B$ (Fig. $13 \mathrm{~A}, \mathrm{~B})$.

A
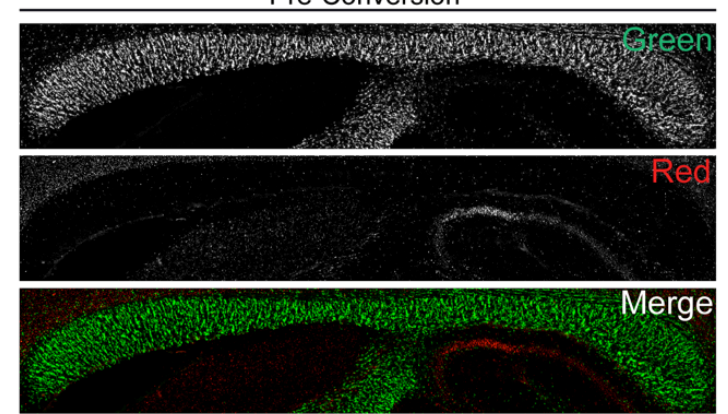

B

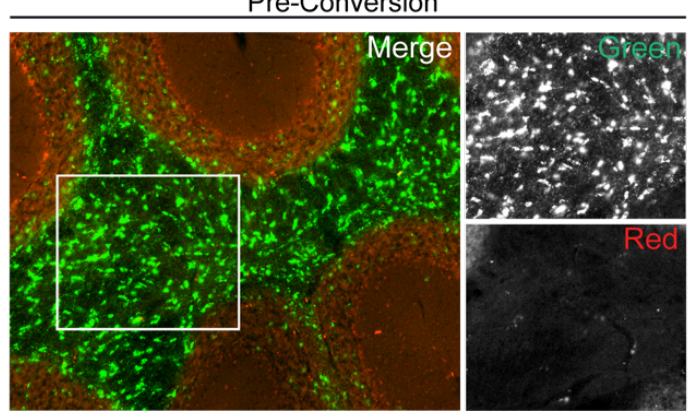

Post-Conversion

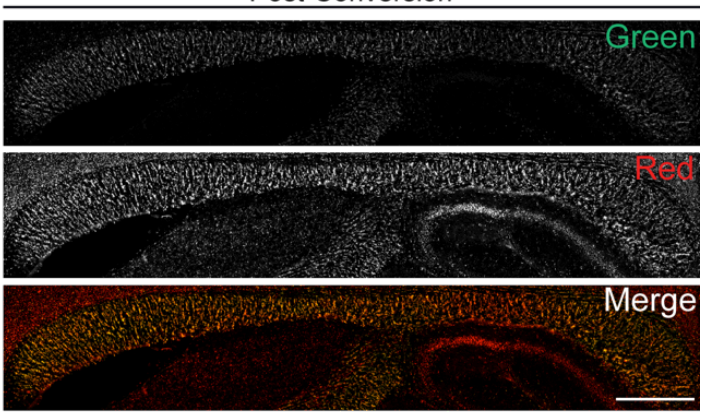

Post-Conversion

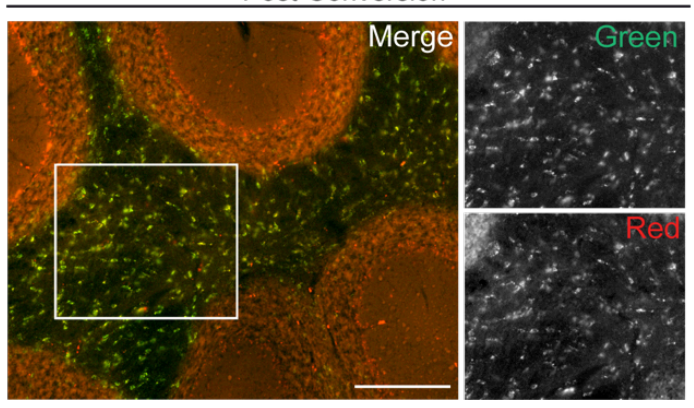

Fig. 13: Photoconversion of vibratome sections from Cnp-mEos2-PTS1 mouse brains The corpus callosum (A) and the cerebellum (B) are depicted before irradiation with $\approx 370 \mathrm{~nm}$ light (left). Red fluorescence is basically absent except for typical, but weak autofluorescence. After photoconversion the green signal is reduced in favor of red fluorescence (right). Insets show magnification of cerebellar white matter. Scale Bar: $200 \mu \mathrm{m}$ 


\subsubsection{Cnp-mEos2-PTS1 expression in oligodendrocytes is not toxic}

High amounts of transgene expression can pose a challenge to organisms. To rule out possible toxic effects of the substantial amount of mEos2 in peroxisomes, 12-month-old Cnp-mEos2-PTS1 transgenic animals were analyzed for cell degeneration and gliosis. The amount of apoptotic cells as observed by Hematoxylin and eosin (H\&E) staining and terminal transferase dUTp nick end labeling (TUNEL) was unaltered between WT and transgenic animals (not shown). Astrogliosis and microgliosis are often early responses to CNS damage (Burda \& Sofroniew 2014). Neither astrocytes (GFAP) nor microglia (IBA1) showed differences in morphology, density or distribution as observed by immunohistochemical analysis in different brain areas (Fig. 14). Cage behavior was observed at regular intervals and did not show abnormalities up to 12 months of age (not shown).

A

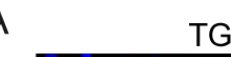
TG WT

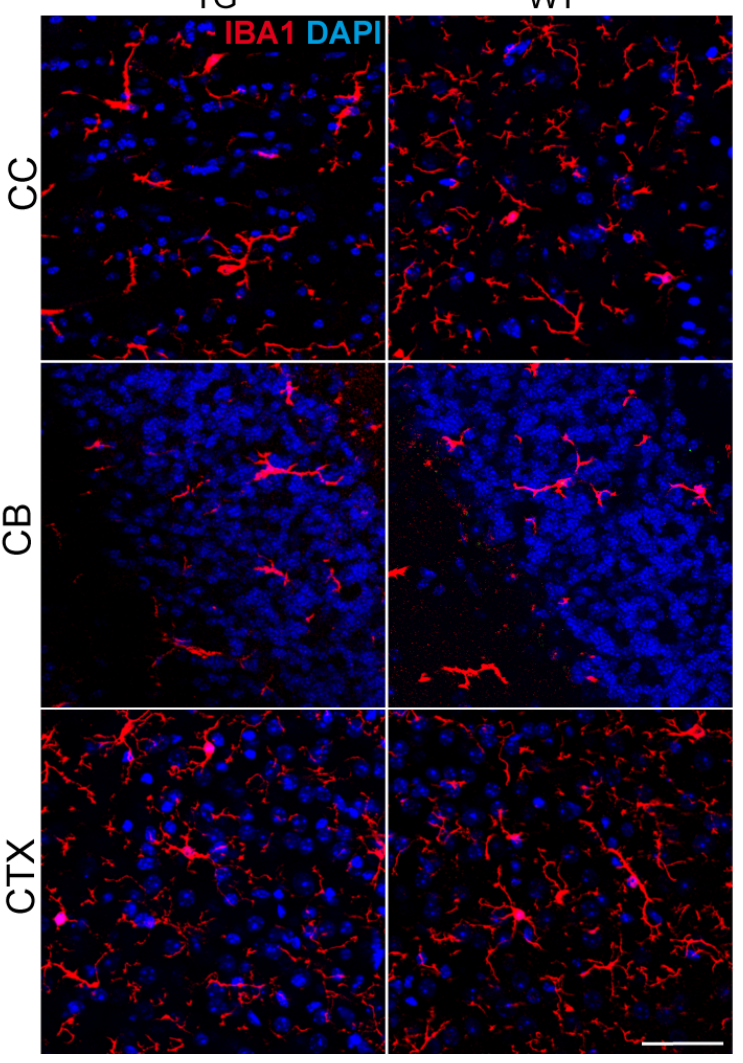

B

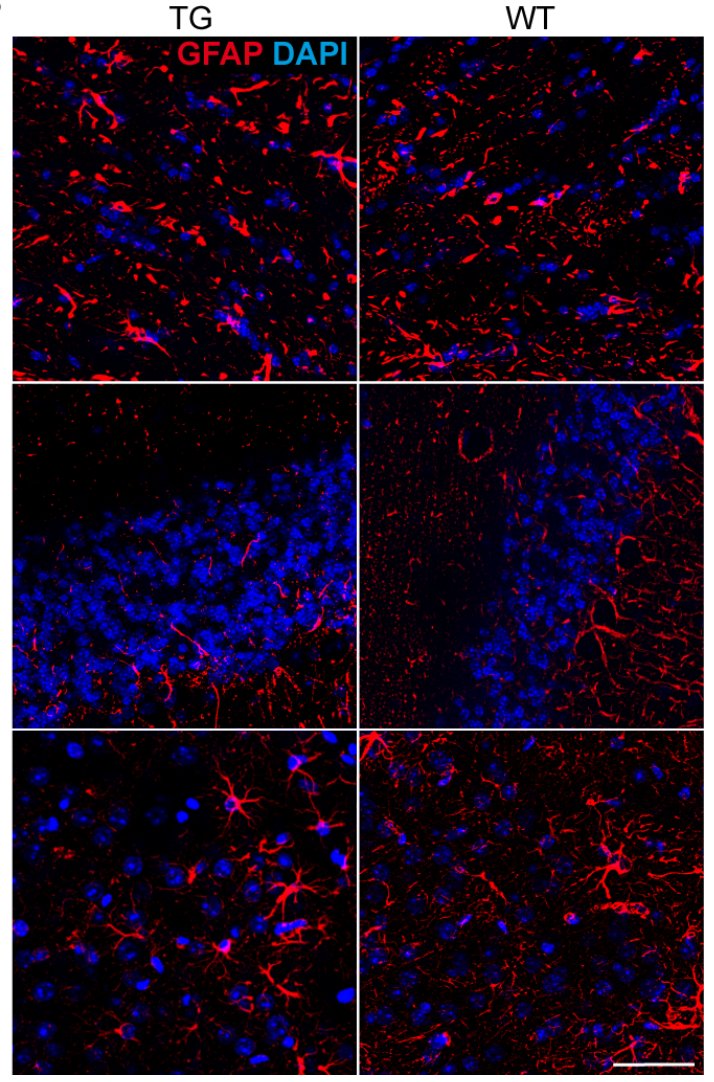

Fig.14: 12-month-old Cnp-mEos2-PTS1 mice show no signs of reactive gliosis

Visualization of the microglial surface marker protein IBA1 (A) and the astroglial fibrillary acid protein GFAP (B) by immunofluorescent labeling reveals no differences between WT and transgenic mice regarding distribution, morphology or expression in the corpus callosum (top), the cerebellum (middle) and the cortex (bottom). Scale bars: $50 \mu \mathrm{m}$. 


\subsection{Reduced peroxisomal abundance and slowed peroxisomal turn-over in $\operatorname{tg} \mathrm{Cnp} \mathrm{Cre}^{\mathrm{CWt} *} \mathrm{Mfp}^{-/-}$mice}

Reduction in peroxisomal abundance has been observed in human skin fibroblasts from patients with impaired $\beta$-oxidation (Chang et al. 1999). To investigate whether

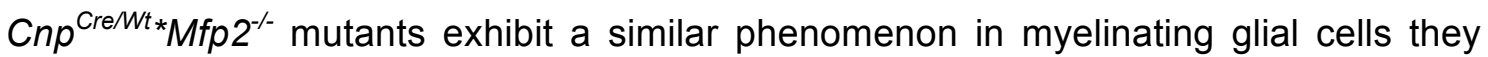
were crossbred to Cnp-mEos2-PTS1 mice (hereafter referred to as $\operatorname{tgCnp}{ }^{\mathrm{Cre} / W t_{*}} \mathrm{Mfp}^{-/-}$

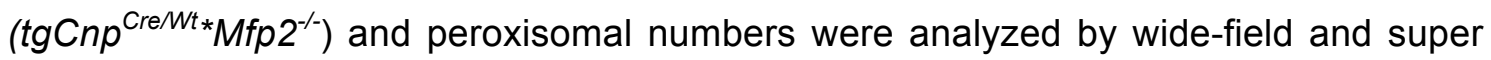
resolution microscopy. Moreover, the employment of a photoconvertible fluorescent protein enabled pulse-labeling of peroxisomal populations to investigate whether peroxisome turnover is altered due to impaired peroxisomal $\beta$-oxidation.

\subsubsection{Reduced peroxisomal abundance in $\operatorname{tg} \mathrm{Cnp}^{\mathrm{Cre} / \mathrm{Wt} *}{ }^{*} \mathrm{Mfp}^{2-/}$ mice}

Vibratome sections of PFA-fixed brains from 1 month (juvenile), 2 months (young adult) and $\geq 16$ months (aged) old animals were taken and nuclei were stained using ToPro3. Z-stacks were acquired on a widefield microscope and oligodendroglial peroxisomes were quantified using Imaris. ToPro-3 positive nuclei associated with mEos2 positive peroxisomes were considered oligodendrocytes. Peroxisome number per oligodendrocyte was unaltered at 1 month of age (Fig. 15A), but already significantly reduced at 2 months and further decreased at 16 months to less than $50 \%$ of Cnp-mEos2PTS1*Mfp ${ }^{\text {flox/flox }}$ (hereafter termed CTR) levels (Fig. 15B). In addition, 16-month-old mutants revealed a tendency to fewer mEos2 positive oligodendrocytes (Fig. 15C), together resulting in a peroxisomal density resembling only $1 / 3$ of control levels. Juvenile CTR mice exhibited more peroxisomes than young adult mice, but overall peroxisome numbers remained unaltered between young adult and aged CTR mice (Fig. 15D). Strikingly, the reduction of peroxisomes in the cellular periphery of oligodendrocytes was even more pronounced than that of the perinuclear organelles in $\operatorname{tg} C n p^{C r e / W t}{ }_{*} M f p 2^{-/}$. This was determined using an automated process to distinguish somatic (within a $4 \mu \mathrm{m}$ radius from the nucleus; Fig. 15E; depicted in green) from peripheral peroxisomes (more than $4 \mu \mathrm{m}$ distance from the nucleus; Fig. 15E; depicted in yellow). Already at 1 month a tendency to less peripheral peroxisomes was observed, at 2 months half of the peripheral peroxisomes had disappeared, and at 16 months only $20 \%$ compared to CTR were left. The strong reduction of peripheral peroxisomes might not only affect myelin maintenance, but as well have indications for the putative axonal support by myelin peroxisomes. 
A
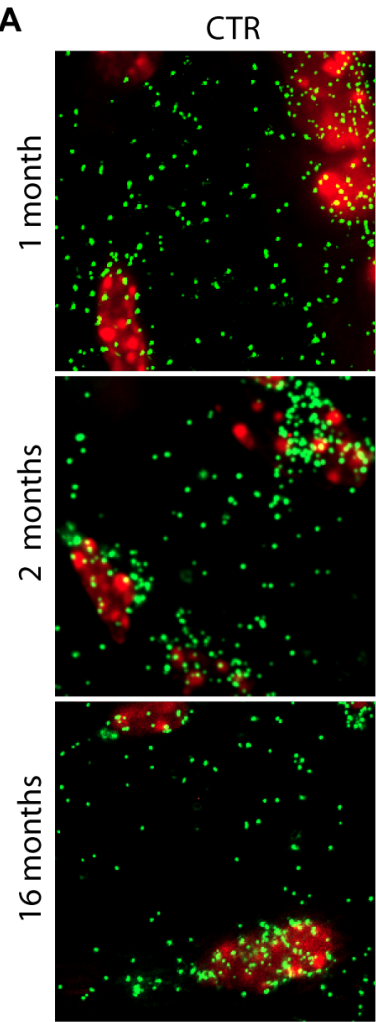

E
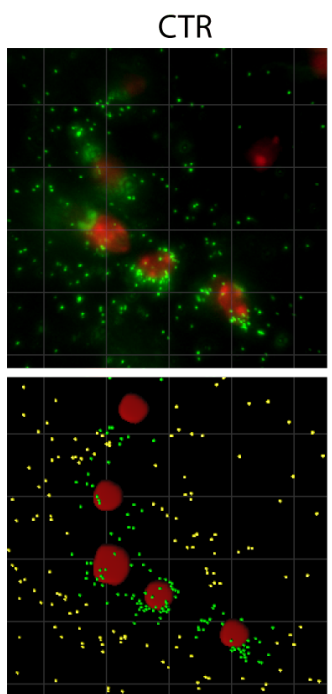

$\operatorname{tg} C$ np Cre $N$ t $* M f p 2 \%$
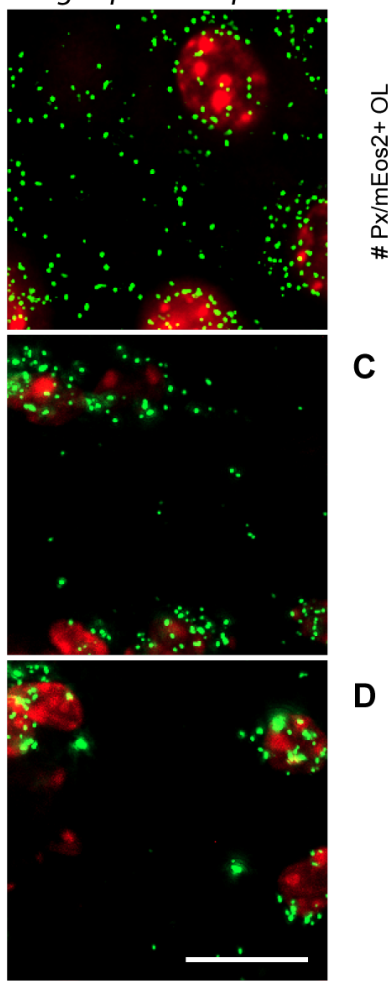

$\operatorname{tg} C n p^{C r e / W t *} M f p 2^{-/-}$
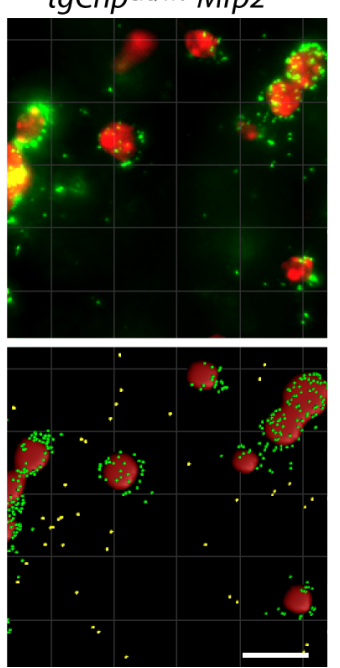

B

C

D

$\mathbf{F}$
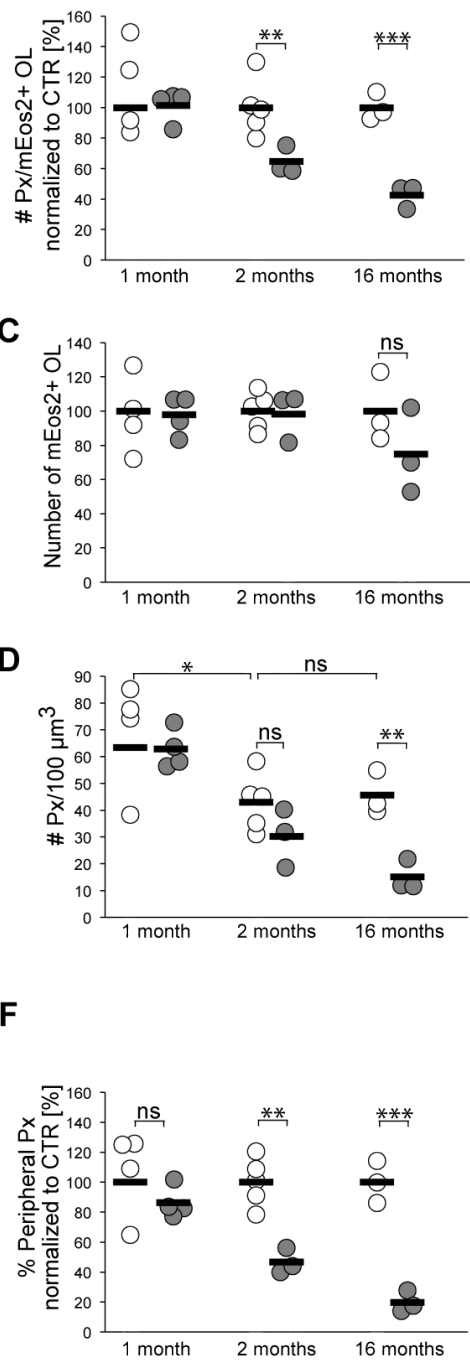

○ CTR

- tgCnp ${ }^{C r e / w t * M f p 2^{-1-}}$

Fig. 15: Reduction of peroxisomes in tgCnp ${ }^{\mathrm{Cre} / \mathrm{t}_{*}} \mathrm{Mfp}^{-{ }^{--}}$mutants

(A) Vibratome sections of transgenic CTR and mutant mice show normal numbers of peroxisomes (green) at 1 month, but a decrease of peroxisomes in two and sixteen-month-old animals; Nuclear stain with ToPro-3 (red). (B) Quantification of peroxisomes per oligodendrocyte prove a significant reduction of about $25 \%$ in 2 months, and more than $50 \%$ in $\geq 16$-month-old mutants. (C) Aged tgCnp ${ }^{\mathrm{Cre}^{*} \mathrm{~N}_{*}} \mathrm{Mfp}^{-{ }^{-/}}$animals show a tendency to less mEos2 positive oligodendrocytes. (D) The combination of fewer mEos2+ oligodendrocytes and less peroxisomes per cell results in a total decrease of more than $2 / 3$ of peroxisomes in $\geq 16$-month-old mutants per $100 \mu \mathrm{m}^{3}$. (E) Examples for the quantification of peroxisomes and the subdivision into somatic peroxisomes (within a $4 \mu \mathrm{m}$ radius from the nucleus; depicted in green) and peripheral peroxisomes ( $>4 \mu \mathrm{m}$ away from the nucleus; depicted in yellow) in Imaris (bottom). The corresponding fluorescent images from $\geq 16$-month-old animals are shown above. (F) Quantification of peripheral peroxisomes shows a decrease of about $80 \%$ in mutants. (CTR $n \geq 3$, mutant $n \geq 3$; Mean + SD). Scale bar: 10 $\mu \mathrm{m}$. 
Histological abnormalities and reactive gliosis in aged $\mathrm{Cnp}^{\mathrm{Cre} / \mathrm{Wt}}{ }^{*} \mathrm{Mfp} 2^{-/-}$mice were most prominent in the frontal $\mathrm{CC}$. The caudal CC showed no obvious pathology (see Fig. $4 \mathrm{~A}$ ). To analyze whether peroxisomal changes were confined to brain areas with severe pathology, vibratome brain sections of 2 and $\geq 16$-month-old $\operatorname{tgCnp}{ }^{\mathrm{Cre} / W t}{ }^{\mathrm{Mfp}} 2^{-/-}$ and appropriate CTR mice were analyzed with respect to peroxisome number and distribution (Fig. 16A).

Quantification of images acquired by wide-field microscopy revealed that the decrease of peroxisomes per area was equivalent between the frontal and the caudal corpus callosum at both time points in mutants (Fig. 16B). Also reduction of peripheral peroxisomes was comparable. Thus altered peroxisome numbers are not confined to brain regions with overt histological alterations.

A
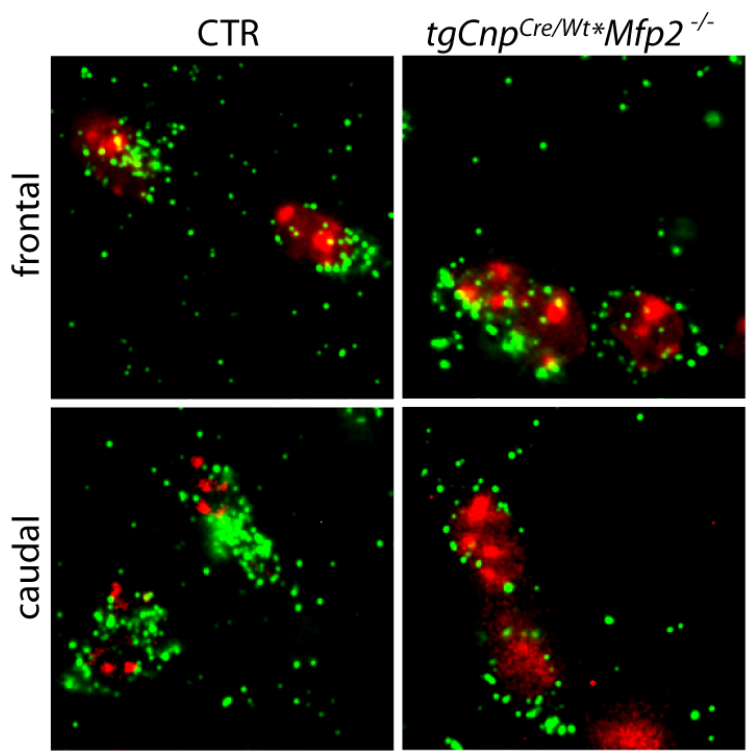

$\operatorname{tg} C n p^{C r e / W t * M f p 2}$

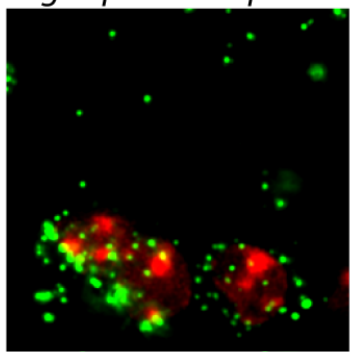

2 months

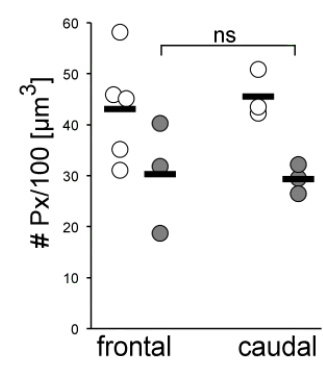

2 months

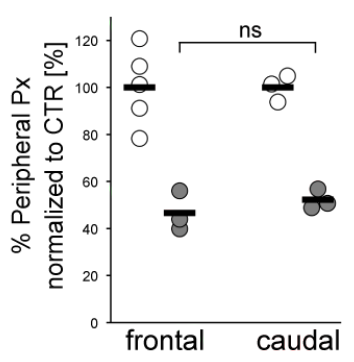

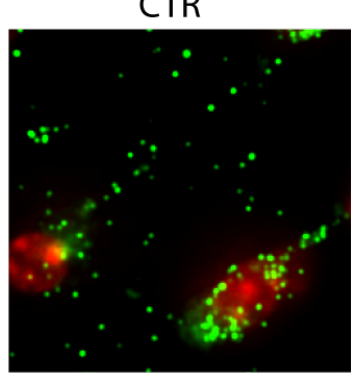

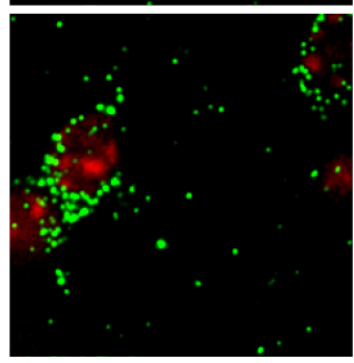

$\geq 16$ months

$\geq 16$ months
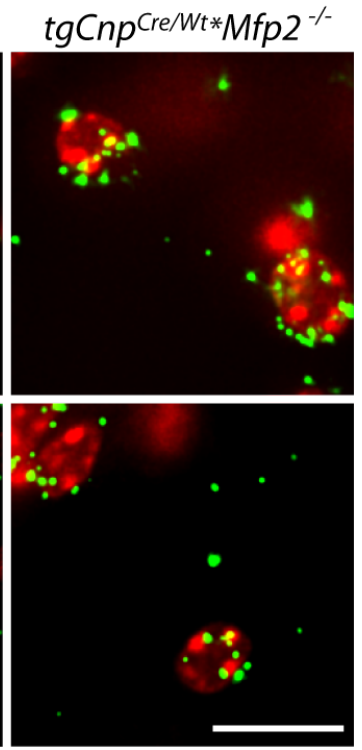

$\longrightarrow$

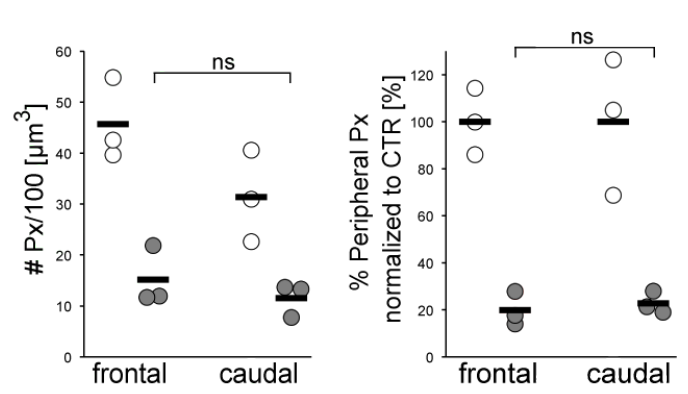

$$
\text { ○ CTR ○ } \operatorname{tgCnp}{ }^{\mathrm{Cre} / w t * M f p 2^{-/-}}
$$

Fig. 16: Reduction of peroxisomes in areas without overt pathology

(A) Vibratome sections of $t g C n p^{\mathrm{Cre} \mathrm{Wt}_{*}} \mathrm{Mfp}^{-{ }^{--}}$animals stained for ToPro-3 (red) show a comparable decrease in peroxisome number (green) between the frontal and the caudal CC. (B) Quantifications confirm no differences in reduction of neither peroxisome number per area nor peripheral peroxisomes between affected and unaffected brain regions. (CTR $n \geq 3$, mutant $n \geq 3$; Mean + SD). Scale bar: $10 \mu m$. 


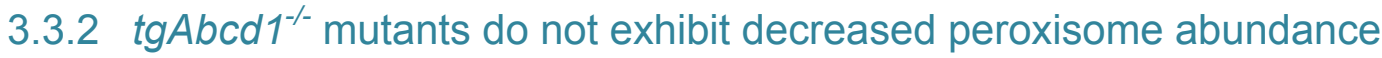

X-linked Adrenoleukodystrophy is caused by mutations in the peroxisomal membrane protein $A B C D 1$, which mediates the import of very long chain fatty acids (VLCFA) into peroxisomes for subsequent degradation by $\beta$-oxidation. About one third of X-ALD patients develop childhood cerebral ALD exhibiting similarities with other disorders caused by peroxisomal $\beta$-oxidation defects. Abcd $1^{-/-}$mice however fail to develop a cerebral phenotype, but only show peripheral nervous system symptoms. The reduction of peroxisomes in MFP2-deficient oligodendrocytes precedes typical neurodegenerative features by several month. Therefore, also $\mathrm{Abcd1^{-/ }}$ mice were analyzed for peroxisomal abundance in brain OL. We analyzed vibratome sections of $\geq 16$-month-old Cnp-mEos2-PTS1*Abcd1 ${ }^{-/}\left(\operatorname{tgAbcd1}{ }^{-/}\right.$; Fig. 17A). The total peroxisome number per area was not altered in $\operatorname{tg} A b c d 1^{-/}$mutants compared to CTR. Furthermore, the quantification of peripheral peroxisomes revealed no reduction.

A

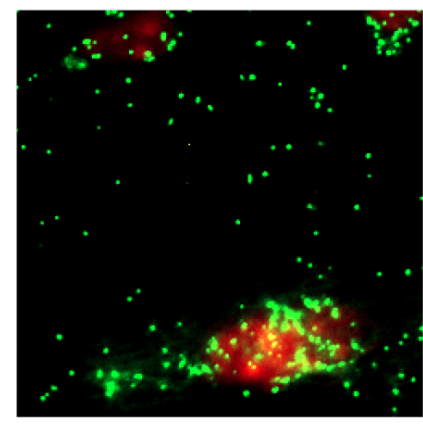

CTR

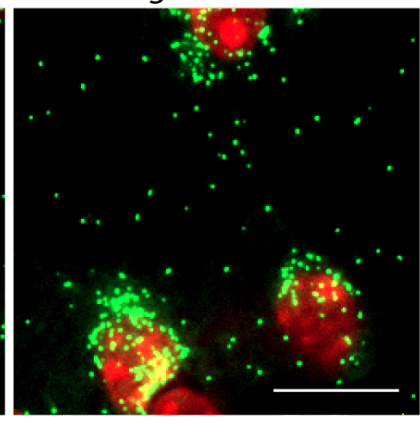

B

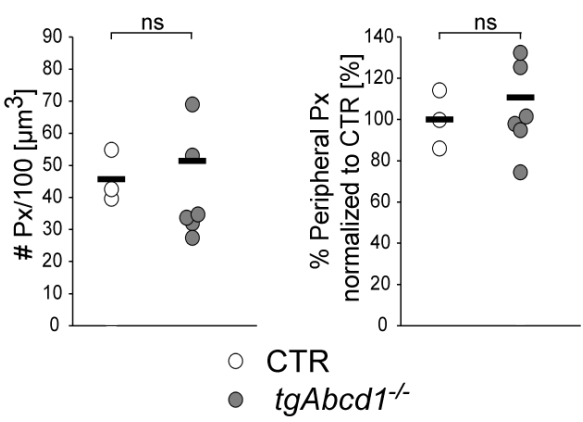

Fig. 17: Peroxisome reduction is not a feature of $\operatorname{tgAbcd1^{-/}}$ mutants

(A) Vibratome sections of $\geq 16$-month-old CTR and $\operatorname{tgAbcd1} 1^{-/-}$mice stained for ToPro-3 (red) show no differences in peroxisomal number (green; B) Quantifications confirm that neither peroxisome number per area nor peripheral peroxisomes are reduced in transgenic Abcd $1^{-/}$compared to CTR animals. (CTR $\mathrm{n} \geq$ 3 , mutant $n \geq 3$; Mean + SD). Scale bar: $10 \mu \mathrm{m}$. 


\subsubsection{MFP2-deficient peroxisomes differ in shape and size}

Peroxisomes in aged transgenic $C n p^{C r e / W t *} M f p 2^{-/-}$mice appeared to differ in size and shape from peroxisomes in corresponding CTRs when analyzed by wide-field microscopy. Since the diameter of brain peroxisomes (0.1- $0.25 \mu \mathrm{m}$; Kovacs et al. 2001) is usually smaller than the diffraction limit of light microscopes we applied the superresolution microscopy technique STED (Stimulated emission depletion; in collaboration with Dr. Katrin Willig) to further characterize peroxisomes in aged mutants. Vibratome sections of immersion fixed brain tissue were taken from CTR and $\operatorname{tgCnp}{ }^{\mathrm{Cre} / W t *} \mathrm{Mfp}^{-/-}$ animals. mEos 2 proved to be suitable for visualization by STED microscopy with regard to quantum yield and photostability (Fig. 18A). Peroxisomal size was increased in

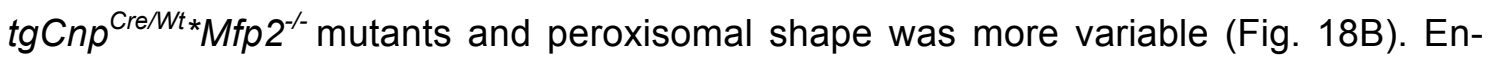
largement of the organelles may be the result of either enhanced growth, or normal growth rates, but lack of subsequent fission or degradation. Hindered fission might also explain reduced sphericity of mutant peroxisomes.

A

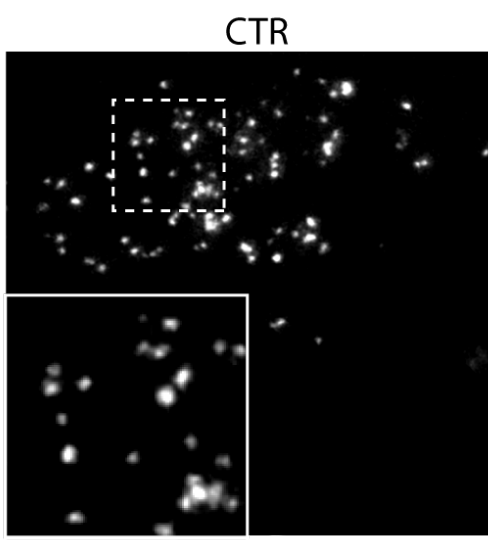

B

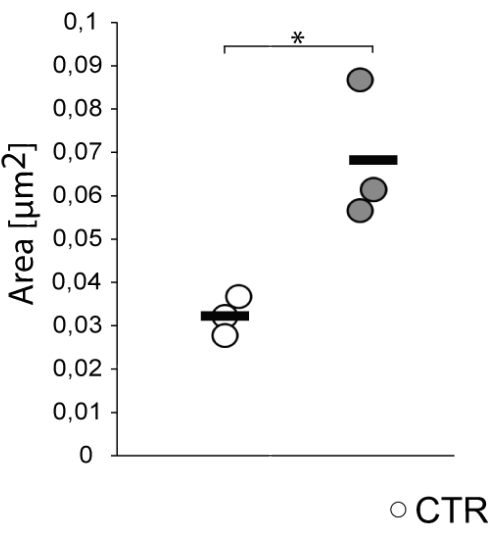

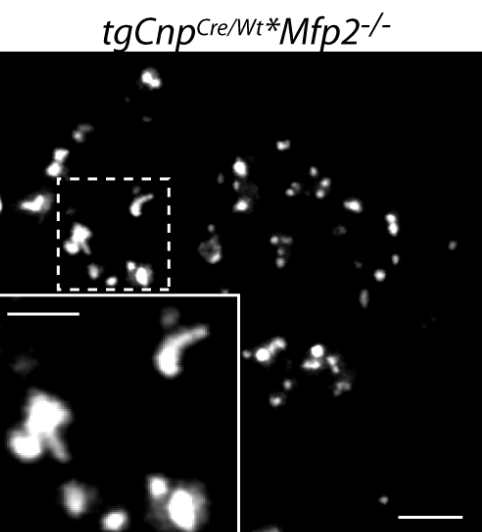

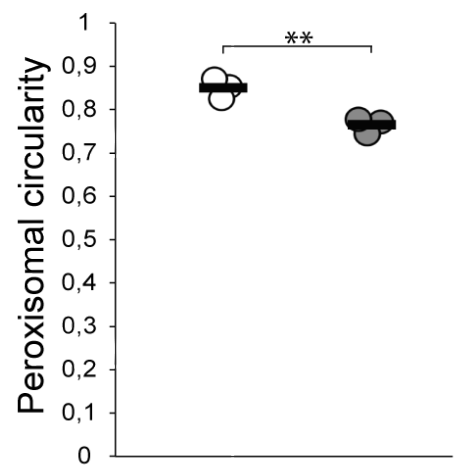

tgCnpCre/wt*Mfp2--

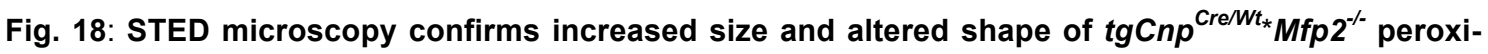
somes

(A) Images of Vibratome sections from $\geq 16$-month-old CTR and mutant animals were acquired by STED microscopy and showed an increase in size of peroxisomes and a more diverse morphology. (B) Quantification with Imaris software confirmed an increase from $0.1 \mu \mathrm{m}^{2}$ in CTR to around $0.13 \mu \mathrm{m}^{2}$ in mutant mice, which also showed decreased circularity. (CTR $n \geq 3$, mutant $n \geq 3$; Mean + SD) Scale bar: $2 \mu m$ and scale bar in magnified image: $1 \mu \mathrm{m}$. 


\subsubsection{Peroxisomal degradation and proliferation in oligodendrocytes}

To analyze peroxisomal turnover in vitro, oligodendrocyte precursor cells (OPCs) were prepared. Individual oligodendrocytes $(\mathrm{OL})$ possessing green mEos2+ peroxisomes were pulse-labeled by $\approx 370 \mathrm{~nm}$ light irradiation, upon which virtually all peroxisomes showed both red and residual green fluorescence (d0; Fig. 19A). The same cells were monitored up to day 8 after photoconversion and peroxisomes were quantified. In CTRs number of peroxisomes exhibiting red fluorescence steadily decreased over time to about $30 \%$ at the final time point compared to $100 \%$ at time point d0 (Fig. 19B). Reduction of pulse-labeled peroxisomes is likely caused by autophagic degradation (pexophagy). In addition, repeated fission events or asymmetrical fission might cause dilution of the red signal to below detection limit. This might lead to artificial loss of detectable red peroxisomes.

Decrease of red peroxisomes went along with a steady increase of peroxisomes exhibiting green, but no red mEos2 fluorescence, which accounted for $76 \%$ of all peroxisomes at $\mathrm{T}_{8}$ (Fig. 19B). Peroxisomes containing green, but no red mEos 2 fluorescence probably arouse by de novo synthesis, but could as well originate from subsequent fission events or asymmetrical fission going along with import of newly synthesized mEos2 protein.

Unlike expected no increase of pulse-labeled peroxisomes due to peroxisomal fission could be observed when looking at the average numbers. However, analysis of individual cells revealed that about $40 \%$ of the OL showed an increase in red fluorescent peroxisomes one or two days after photoconversion (Fig. 19C). This confirms that peroxisomal proliferation via fission occurs in OLs and beyond demonstrates that at least a limited number of fission events can be detected by our assay in CTR OL. Interestingly the reappearance of only green mEos2 positive peroxisomes did not differ in OL with or without peroxisomal fission events (Fig. 19C).

Taken together these results indicate that around $70 \%$ of the total peroxisomal pool in oligodendrocytes is turned over in a time frame of eight days, which corresponds to a peroxisomal half life of about 4.5 days. However, it cannot be clearly differentiated whether the whole organelle or the peroxisomal protein content is turned over. Analysis of OL subpopulations proved the detectability of fission in OLs by our assay and furthermore indicates an independent regulation of fission and de novo synthesis. 
A
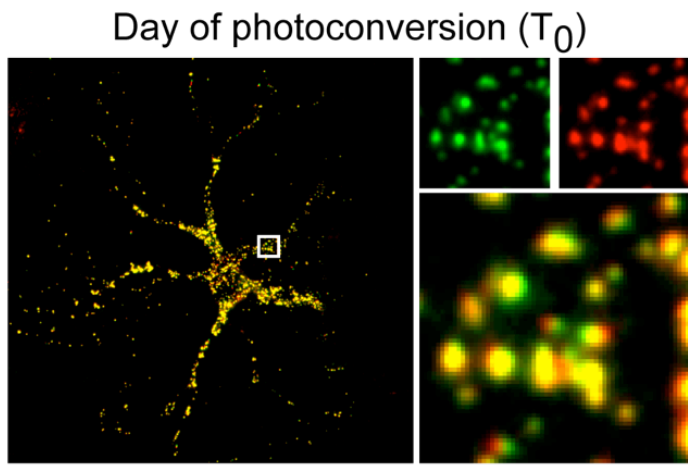

B

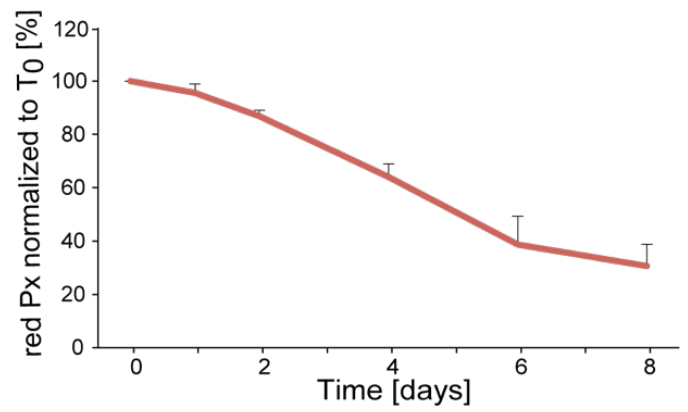

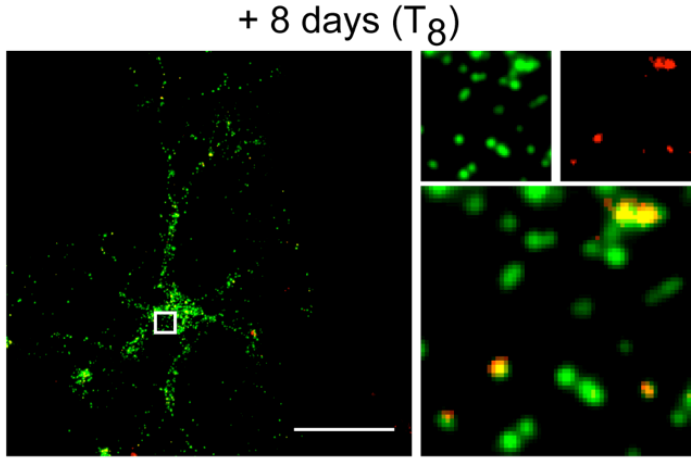

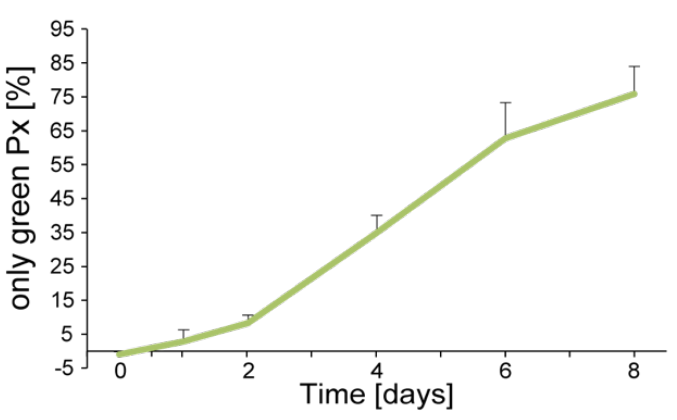

C OLs devided into subopulations with or without (w/o) peroxisomal fission
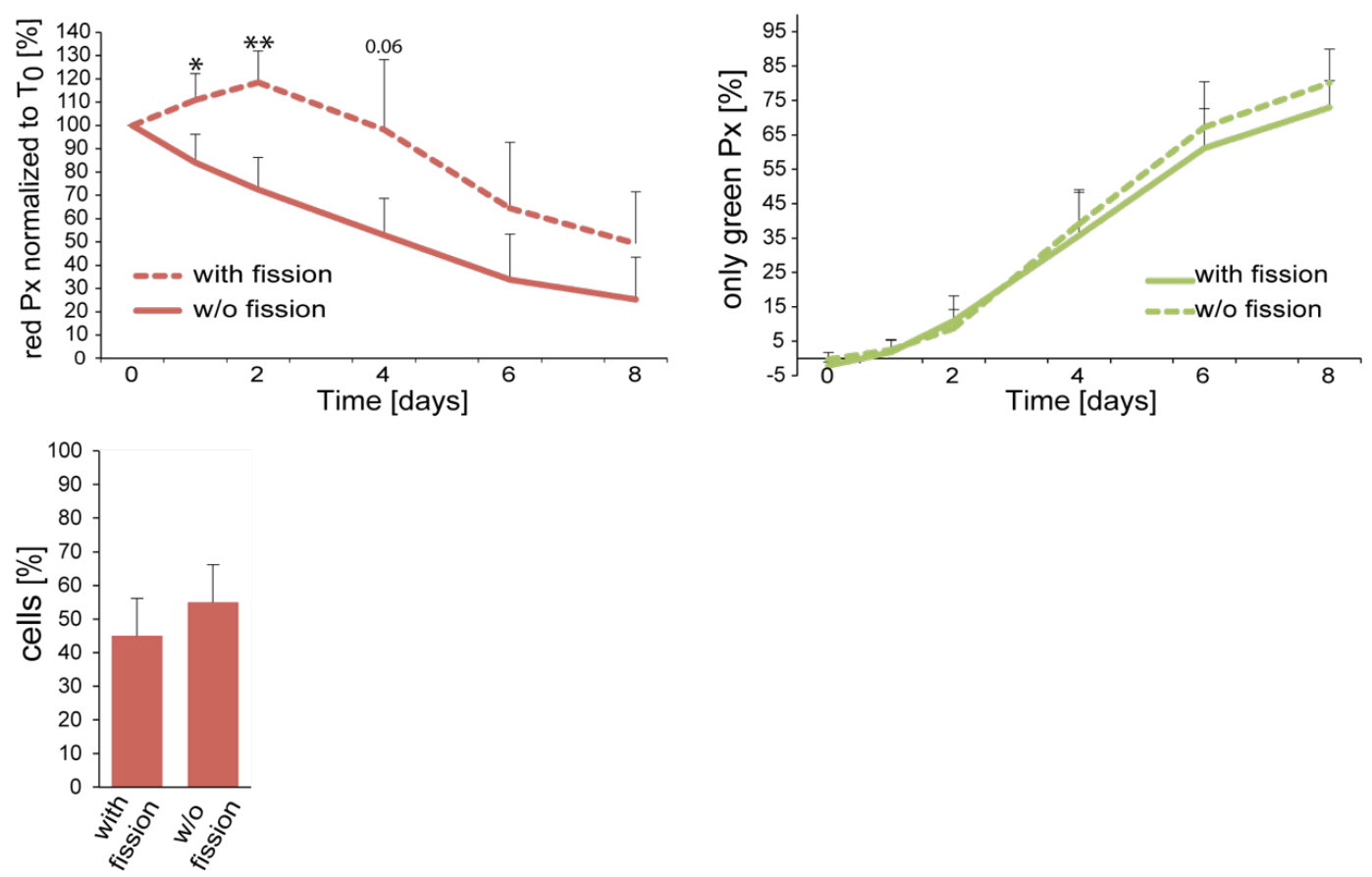

Fig. 19: Peroxisomal turnover in CTR oligodendrocytes

(A) Representative images of a CTR OL immediately after photoconversion ( $T_{0}$; left) and 8 days later $\left(T_{8}\right.$; right) show a marked decrease of peroxisomes exhibiting red mEos2 fluorescence at the later timepoint. Scale Bar: $50 \mu \mathrm{m}$ (B) Quantifications revealed that the number of pulse-labeled peroxisomes decreases rapidly to around $30 \%$ at day 8 , normalized to day 0 , while the number of only green peroxisomes increases steadily to $75 \%$ at day eight. (C) About $40 \%$ of OLs show peroxisomal fission of pulse-labeled peroxisomes (left). The number of newly formed only green peroxisomes increases independently of fission events (right). ( $\geq 3$ independent experiments with $\geq 10$ cells analyzed per experiment; Mean $+S D$ ) 


\subsubsection{Slowed peroxisomal turnover in $\operatorname{tg} \mathrm{Cnp}{ }^{\mathrm{Cre} / \mathrm{Nt} *} \mathrm{Mfp}^{2-1-}$}

The observed peroxisomal alterations in $\operatorname{tgCnp}{ }^{\mathrm{Cre} / W t^{*}} M f p 2^{-/-}$mutants brains cannot be explained by the defect in peroxisomal $\beta$-oxidation. Impairment of the $\beta$-oxidation pathway may however influence peroxisomal turnover indirectly via alterations of peroxisomal biogenesis or degradation. Therefore, OL cultures were generated from CTR and mutant mice and pulse-label experiments conducted as described before. Both, CTR and mutant cells developed sheet-like structures as visualized by staining for Myelin basic protein (MBP; Fig. 20A). Comparable to the in vivo situation, mature

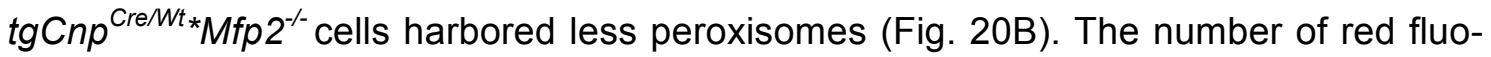
rescent mEos2 peroxisomes was more stable than in CTRs and even increased slightly up to day 2. Eight days after photoconversion around $95 \%$ of pulse-labeled peroxisomes were still detectable, implying impaired degradation of peroxisomes or possibly also a reduced number of fission events in mutant cells (Fig. 20E). Furthermore, the reappearance of peroxisomes containing only green mEos2 fluorescence was markedly reduced in mutant $\mathrm{OL}$, accounting for less than $20 \%$ of all peroxisomes at the last time point analyzed (Fig. 20F). mEos2 fluorescence increased over time indicating continuous protein import into peroxisomes (Fig. 20C). Impaired de novo synthesis, but also less fission events could account for the reduced reappearance of only green peroxisomes. Taken together peroxisomal aging is evidently increased in MFP2-deficient mutants compared to control oligodendrocytes.

$2^{\prime}, 3^{\prime}$-cyclic nucleotide $3^{\prime}$-phosphodiesterase (CNP) accounts for about $4 \%$ of CNS myelin proteins. Heterozygous depletion of $\mathrm{Cnp}$ has been shown to be associated with mild axonal degeneration in aged mice ( $>24$ months; Hagemeyer et al. 2012). Furthermore Cre expression was related to increased oxidative stress and peroxisome proliferation in mice (Xiao et al. 2013). To exclude that reduced Cnp gene dosage or heterozygous Cre expression causes the effects observed in $\operatorname{tgCnp}{ }^{\mathrm{Cre} / \mathrm{Wt} *} \mathrm{Mfp}^{-/-}$cells, Cnp-mEos2-SKL ${ }^{*} C n p^{C r e / W t}$ (hereafter referred to as $\mathrm{tgCnp}{ }^{\mathrm{Cre} / \mathrm{Wt}}$ ) animals were generated. Pulse-labeling experiments in $\mathrm{OL}$ were conducted and peroxisomes quantified on day 2, 4 and 8 after photoconversion. At two days after photoconversion a transient increase in red mEos2 positive peroxisomes in tgCnp ${ }^{\mathrm{Cre} / \mathrm{Wt}}$ animals was observed, possibly due to Cre mediated oxidative stress, that was absent from CTR cells (Fig. 20E). Already at four days after photoconversion the number of pulse-labeled peroxisomes was indistinguishable from CTR cells. The reappearance of newly formed peroxisomes was comparable between $\operatorname{tgCnp}{ }^{\mathrm{Cre} / \mathrm{Wt}}$ and CTR cells (Fig. $20 \mathrm{~F}$ ). $\operatorname{tgCnp}^{\mathrm{Cre} / \mathrm{Wt}}$ showed a high turnover of peroxisomes similar to CTR cells. Hence, neither reduced Cnp gene dosage nor heterozygous Cre expression account for the changes observed $\operatorname{tg} C n p^{\mathrm{Cre} / \mathrm{Wt} *}{ }_{\mathrm{Mfp}} 2^{-/-}$OLs. 


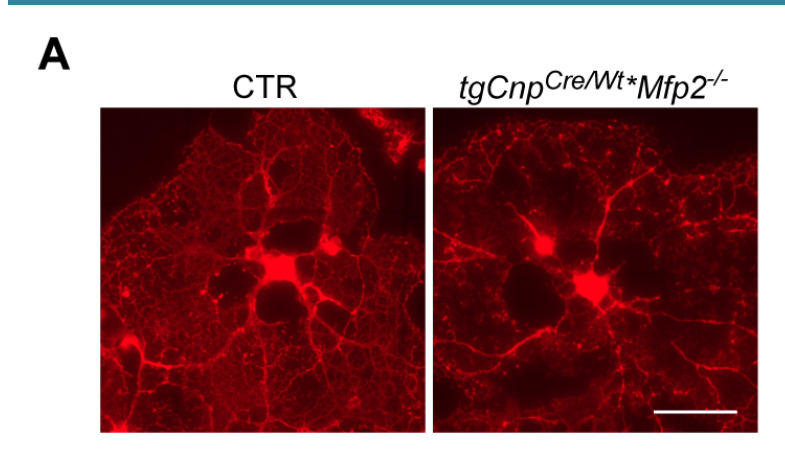

B C

D
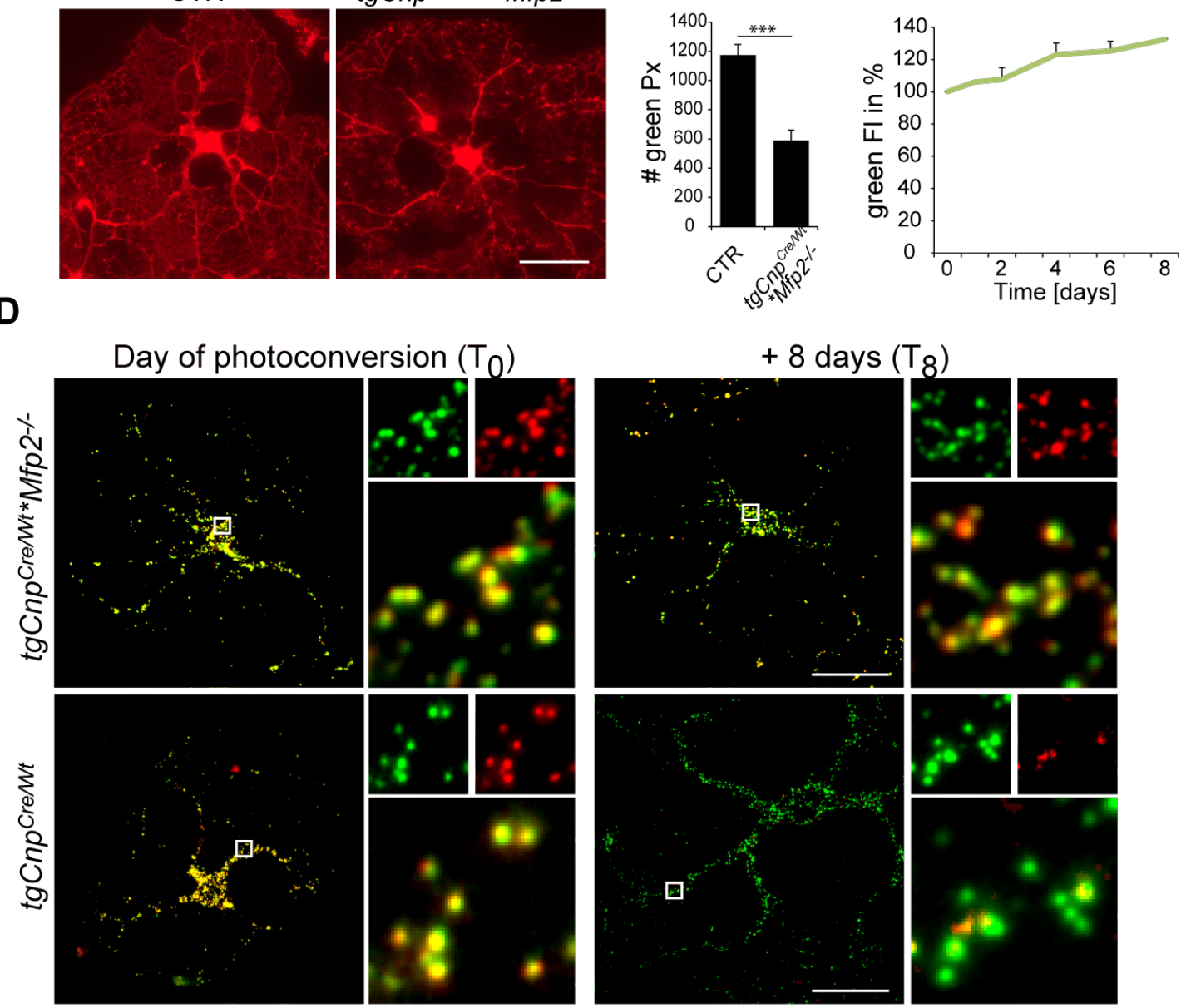

+8 days $\left(T_{8}\right)$

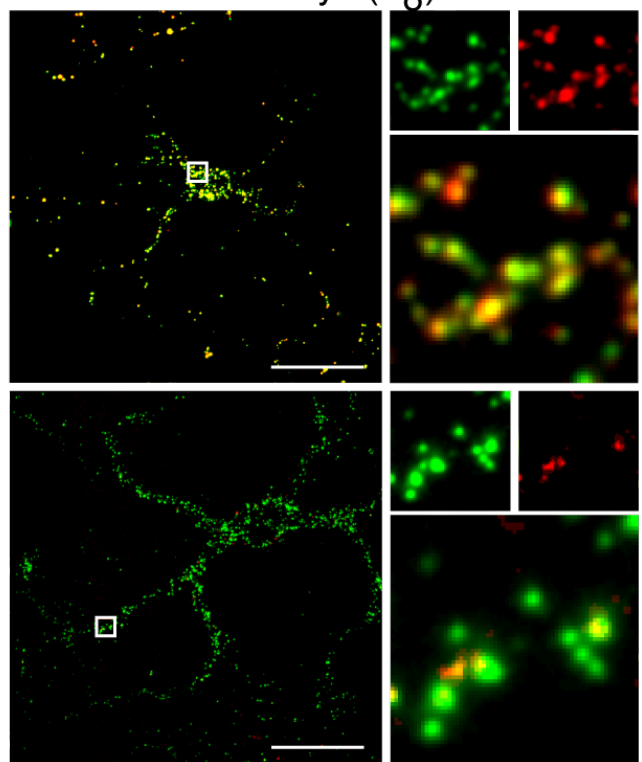

E
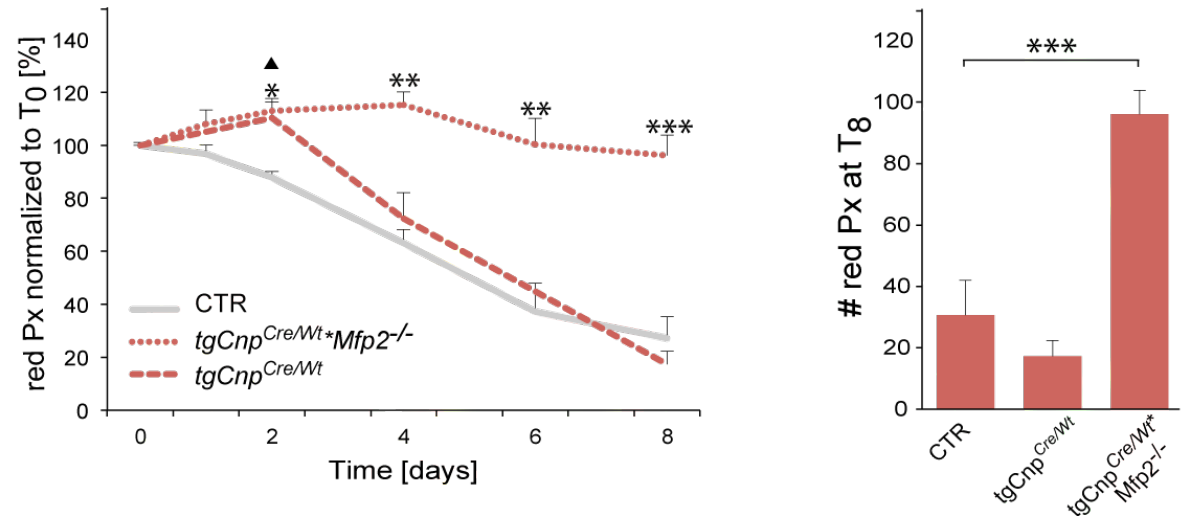

F
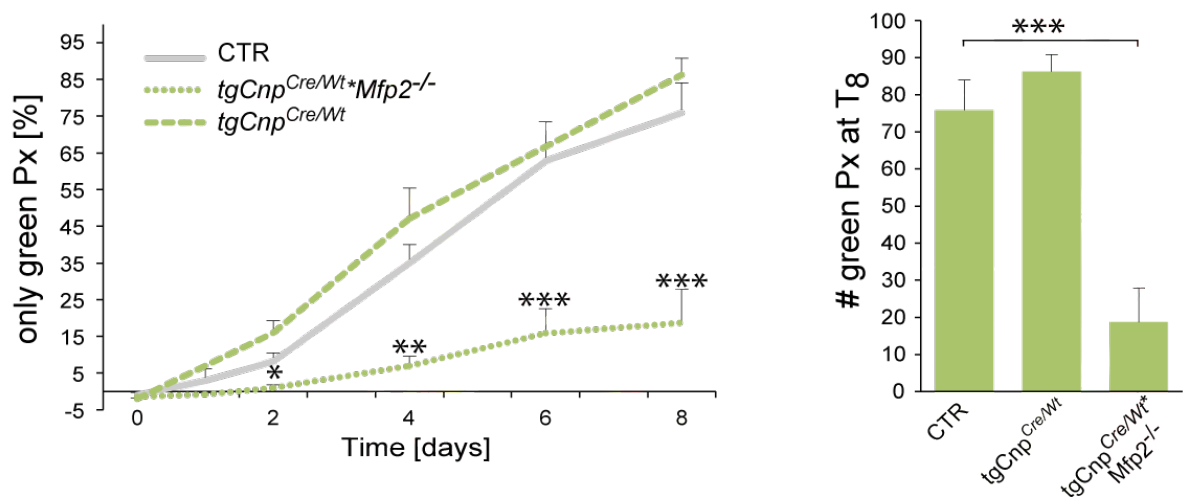


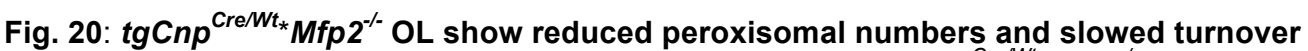

(A) Immunostaining for Myelin basic protein (MBP) in CTR and tgCnp ${ }^{\mathrm{Cre} W t^{*}}{ }^{\mathrm{Mfp}} 2^{-/-}$OLs 8 days after plating demonstrates myelin sheet-like formation. Scale Bar: $50 \mu \mathrm{m}$ (B) Imaris quantification of mEos2 positive peroxisomes shows a decrease in peroxisomal abundance of about $50 \%$ in day 8 mutant OLs. (C) Analysis of intraperoxisomal mEos2 signal in $\operatorname{tgCnp}{ }^{\mathrm{Cre} \mathrm{Wt}_{*}} \mathrm{Mfp}^{-{ }_{-1}}$ reveals constant increase in fluorescence inten-

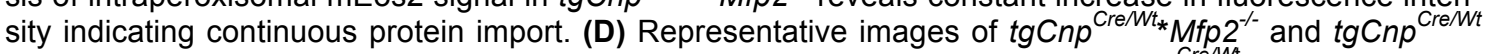
OLs immediately after photoconversion ( $\mathrm{T}_{0}$; left) and 8 days later ( $\mathrm{T}_{8}$; right). $\operatorname{tg} \mathrm{Cnp} \mathrm{cre}^{\mathrm{Cr} t}$ OLs show a decrease of peroxisomes exhibiting red mEos2 fluorescence at $\mathrm{T}_{8}$ comparable to CTRs. In $\operatorname{tgCnp}{ }^{\mathrm{Cre} / \mathrm{Wt}}{ } \mathrm{Mfp}^{-/-}$ the majority of peroxisomes still exhibits both green and red mEos2 fluorescence at $T_{8}$. Scale Bar: $50 \mu \mathrm{m}$ (E) Quantification confirms enhanced stability of the number of pulse-labeled peroxisomes in tgCnp ${ }^{C r e / t_{*}}{ }^{M f p} 2^{-/-}$OLs (96\%), whereas $\operatorname{tgCnp}{ }^{\mathrm{Cre} / W t}(17 \%)$ compare to CTR cells $(27 \%$; triangle indicates Pvalue $<0.05$ in the $\operatorname{tgCnp}{ }^{C r e / W t}$ cells; F) The reappearance of peroxisomes exhibiting only green, but no red mEos2 fluorescence is reduced in tgCnp ${ }^{\mathrm{Cre}^{\prime} W t_{\star}} \mathrm{Mfp}^{-/-}(19 \%)$, compared to $\operatorname{tgCnp}{ }^{\mathrm{Cre} / W t}(86 \%)$, and CTRs (76\%). ( $\geq 3$ independent experiments with $\geq 10$ cells analyzed per experiment and genotype; Mean + SD).

\subsubsection{3-Methyladenine treatment reduces autophagic degradation and peroxi- somal biogenesis}

Pulse-label experiments in CTR oligodendrocytes showed steady reduction of pulselabeled red mEos2-positive peroxisomes. To investigate whether this is mainly caused by autophagy or by fission, peroxisomal degradation was inhibited by application of 3Methyladenine (3-MA), an autophagy blocker (Iwata et al. 2006; Kaushik et al. 2008). Peroxisome stability was markedly increased by 3-MA treatment as depicted in (Fig. 21A). Quantification of pulse-labeled peroxisomes revealed a nearly stable number of labeled peroxisomes up to day 4 and a final decrease by only $30 \%$ compared to $70 \%$ in untreated CTRs (Fig. 21B). The impaired degradation of peroxisomes upon 3-MA treatment indicates that the majority of peroxisomes in CTR cells is degraded via macroautophagy. Other degradative pathways, but also dilution of the photoconverted protein by fission, photo-bleaching, or intraperoxisomal protein degradation may contribute to the residual loss of labeled peroxisomes in 3-MA and CTR cells.

Remarkably 3-MA treatment also drastically reduced the reappearance of only green peroxisomes to about $20 \%$ at the final time-point compared to more than $75 \%$ in CTR OL (Fig. 21B). This suggests a coupling of peroxisomal biogenesis to autophagic degradation, though it cannot yet be excluded whether 3-MA has a direct effect on peroxisomal biogenesis. These observations may imply that in $t g C n p^{C r e / W t *}{ }^{*} \mathrm{Fp}^{-{ }^{--}}$oligodendrocytes impaired organelle degradation might precede and cause slowed peroxisomal biogenesis. 
A
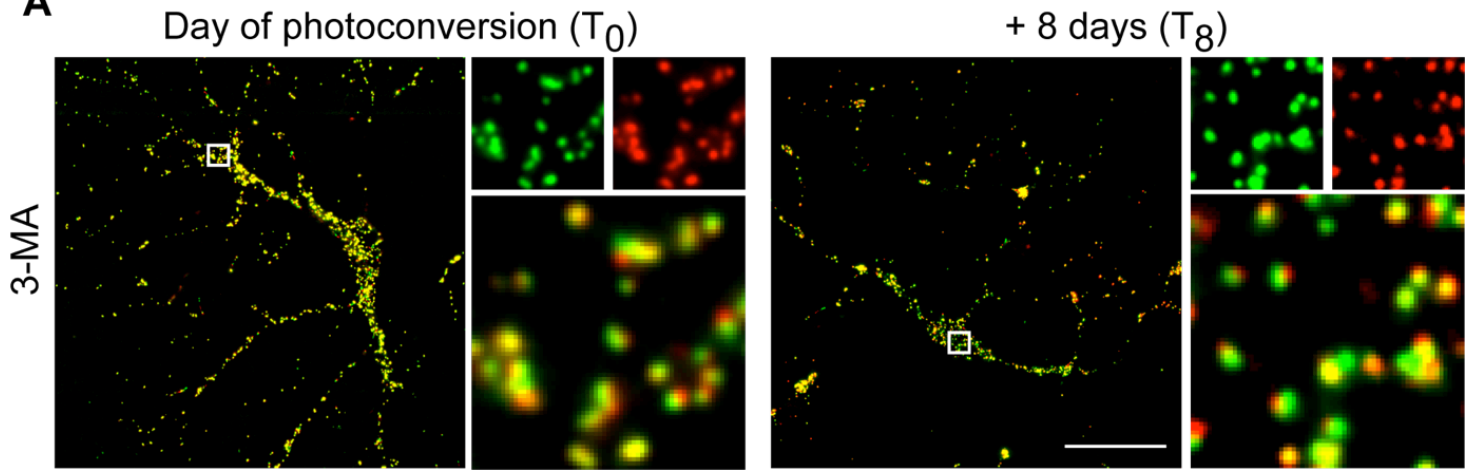

B
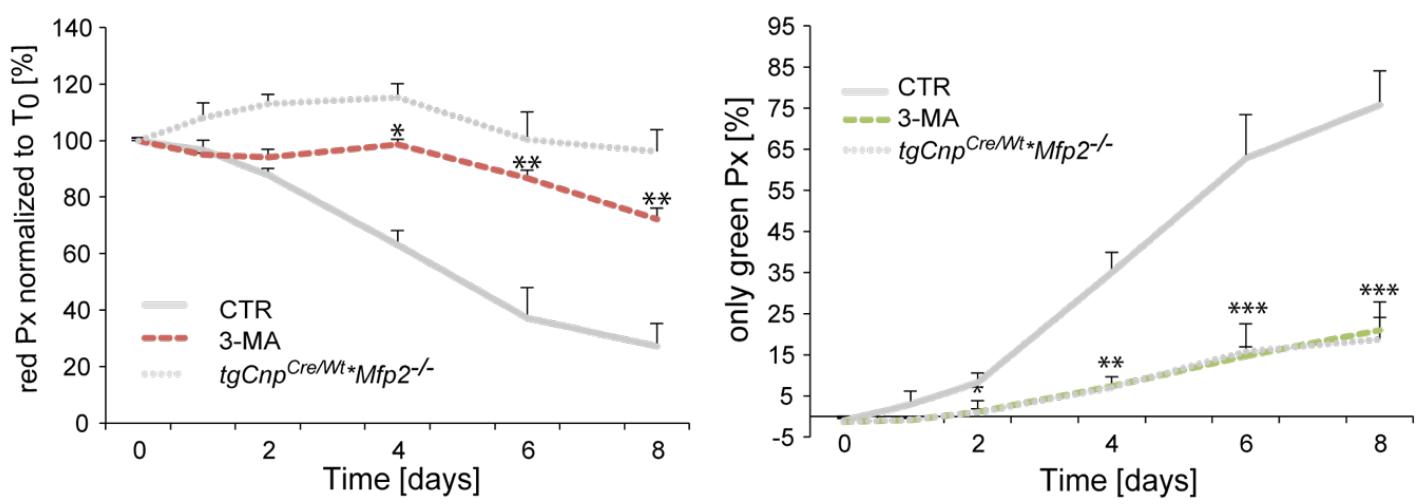

Fig. 21: Reduced autophagic degradation also impairs peroxisomal biogenesis

(A) Representative images of CTR OLs treated with $10 \mathrm{mM}$ 3-Methyladenin (3-MA). Images acquired immediately after photoconversion ( $\mathrm{T}_{0}$; left) and 8 days later $\left(\mathrm{T}_{8}\right.$; right) show a high amount of peroxisomes exhibiting both green and red mEos2 fluorescence at $\mathrm{T}_{8}$ resembling $\operatorname{tgCnp^{Cre}/\mathrm {Wt}_{\star }} M f p 2^{-/}$. Scale Bar: $50 \mu \mathrm{m}$. (B) Quantification confirms enhanced stability of the number of pulse-labeled peroxisomes in 3-MA treated OLs $(72 \%)$ compared to CTRs $(27 \%)$. Reappearance of peroxisomes exhibiting only green, but no red mEos2 fluorescence is impaired in 3-MA treated cells $(21 \%)$ compared to CTRs $(76 \%)$. $(\geq 3$ independent experiments with $\geq 10$ cells analyzed per experiment; Mean + SD). 


\section{Discussion}

Peroxisomal biogenesis disorders (PBDs) are caused by mutations in peroxins that result in the loss of peroxisomal function. Patients often exhibit severe central nervous system and hepato-renal symptoms. The former comprises cortex and cerebellum malformations, peripheral neuropathy, and inflammatory demyelination (Waterham 2016). Interestingly, patients carrying mutations only in single enzymes or transporters involved in peroxisomal $\beta$-oxidation often show common hallmarks with PBDs unlike patients with impaired peroxisomal a-oxidation or plasmalogen synthesis, who present with a distinct clinical picture. This indicates a common pathomechanism for both types of disorders, those caused by impairment of peroxisomal b-oxidation and those caused by peroxisomal biogenesis defects. Further indications for an analog disease etiology derive from mice lacking peroxisomal function from oligodendrocytes $\left(\mathrm{Cnp}{ }^{\mathrm{Cre} / \mathrm{Wt} *} \mathrm{Pex} 5^{-/-}\right.$ ). These mutant mice exhibit inflammatory subcortical demyelination reminiscent of the cerebral pathology of patients affected with cerebral ALD, which is caused by mutations in a peroxisomal transporter for very long chain fatty acids. To analyze whether certain cell types play a decisive role for neurodegeneration, peroxisomal function was depleted from astrocytes (Gfap ${ }^{\mathrm{Cre} / W t *} P \operatorname{ex} 5^{-/}$), neurons $\left(\mathrm{Nex}{ }^{\mathrm{Cre} / \mathrm{Wt} *} \mathrm{Pex} 5^{-/}\right)$and oligodendrocytes $\left(C n p^{C r e / W t *} P e x 5^{-/}\right)$. While Gfap ${ }^{C r e / W t *} P e x 5^{-/-}$and $N e x{ }^{C r e / W t *} P e x 5^{-/-}$exhibited no overt phenotype $C n p^{C r e / W t *} P e x 5^{-/}$showed demyelination, axonal degeneration and reactive gliosis similar to mice lacking peroxisome function from all brain cells (Nestin${ }^{C r e} /{ }^{*}{ }^{*} P e x 5^{-/}$) albeit ameliorated. This indicates that oligodendroglial peroxisomes play a crucial role for myelin maintenance and axonal integrity. To investigate the role of pe-

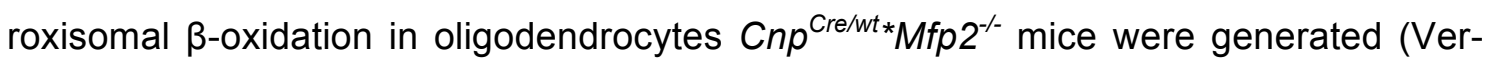
heijden et al. 2013). Here, we analyzed aged ( $\geq 16$ month) $C n p^{C r e / w t *} M f p 2^{-/-}$mice to elucidate, whether prolonged impairment of $\beta$-oxidation might result in a late-onset brain pathology. 


\section{1 $\mathrm{Cnp}^{\mathrm{Cre} / \mathrm{Wt}_{*}} \mathrm{Mfp} 2 \%$ display a late-onset subcortical demye- lination reminiscent of mice with peroxisomal biogenesis defects in oligodendrocytes}

Mutations in enzymes or transporters of the peroxisomal $\beta$-oxidation, such as ACOX1 or $A B C D 1$, often exhibit rather mild phenotypes in mice with mainly hepato-renal problems, but no overt brain pathology. The corresponding mutations in humans show a clinical presentation similar to PBD patients, but with later onset, indicating that the short life-span of mice possibly impedes the development of neurodegeneration. The multifunctional protein 2 (MFP2) shows the most severe pathology throughout peroxisomal $\beta$-oxidation defects in man. This is reflected in MFP2-deficient mice, which exhibit a neurodegenerative pathology including cerebellar atrophy and inflammation. Unlike patients, mutant mice do not develop inflammatory cerebral demyelination before they perish around 6 months of age, likely due to the peroxisomal defects in liver (Baes 2000; Huyghe et al. 2006). Nestin ${ }^{\star} M f p 2^{-/-}$mice lacking MFP2-function from all brain cells, except microglia, were developed to concentrate on the role of peroxisomal $\beta$-oxidation in the brain uncoupled from liver defects. These mice also show a neurodegenerative pathology, albeit with reduced neuroinflammation and enhanced lifespan compared to conventional MFP2-knockout mice. This milder brain pathology may be attributed to the preservation of peroxisomal $\beta$-oxidation in microglial cells in this mouse model.

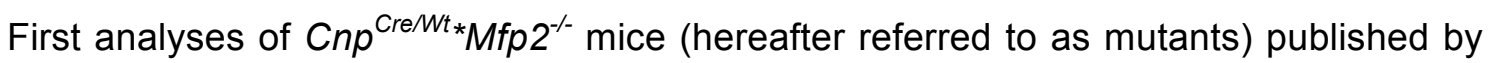
Verheijden et al. showed neither overt phenotypic alterations nor structural myelin changes compared to controls in animals $\leq 12$ month of age (Verheijden et al. 2013). This indicates that $\beta$-oxidation in oligodendrocytes is not crucial for initial myelination. This is in line with observations from $C n p^{\mathrm{Cr} / W t *} P e x 5^{-/}$mice lacking peroxisomal function from oligodendrocytes completely, but displaying normal myelin until the age of 2 months. When analyzing $\mathrm{Cnp}^{\mathrm{Cre} / W{ }_{*}} \mathrm{Mfp}^{-/-}$mice, we detected an abnormal loss in weight gain starting around 12 months, indicating a possible onset of disease. Motorical impairment as evidenced by measuring behavioral tasks started around 15 months of age. Histologic and electron microscopic analysis at $\geq 16$ months revealed subcortical demyelination in mutants, starting in the frontal corpus callosum (genu of corpus callosum). This was accompanied in the same brain region by signs of axonal pathology, i.e. abnormal swellings and reduced axonal abundance. Furthermore, reactive gliosis and infiltration of peripheral CD3-positive T-lymphocytes were revealed specifically in demyelinating lesion sites. Histological abnormalities were mainly concentrated in 
the genu of corpus callosum, which is also primarily affected in mice without functional

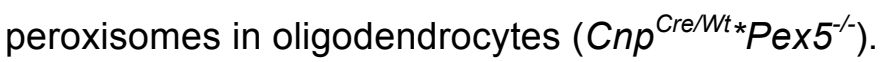

The question remains as to what causes region specific onset of pathology in the

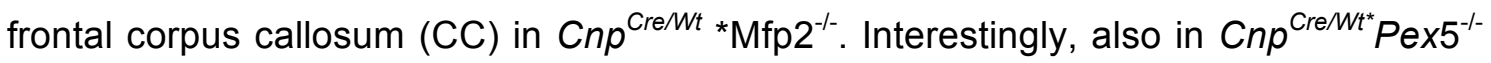
mutants the genu of corpus callosum is mainly affected. Similarly, cuprizone treatment in rodents showed most severe demyelination in the CC and CALD patients often exhibit initial demyelinating lesions in the caudal CC (Engelen et al. 2012; Steelman et al. 2012). So far the cause for the region specificity remains enigmatic, but an activity dependency might be envisioned. The $\mathrm{CC}$ has been shown to utilize higher amounts of glucose in rats, a cross species comparison could not confirm this however (Sokoloff 1977; Karbowski 2007).

It is not resolved yet, which peroxisomal $\beta$-oxidation substrates are important with respect to brain pathology in MFP2-deficiency. This is possibly further complicated by the overall limited knowledge on $\beta$-oxidation substrates in the CNS (Baes \& Van Veldhoven 2012). However, a biochemical hallmark of MFP2-deficiency is the accumulation of several peroxisomal $\beta$-oxidation substrates such as VLCFA, bile acid intermediates, and pristanic acid (Ferdinandusse et al. 2006). In contrast, docosahexaenoic (DHA), the most abundant polyunsaturated fatty acid in the brain, is a product of peroxisomal $\beta$-oxidation, which is reduced when peroxisomal $\beta$-oxidation is impaired. DHA is a precursor for bioactive molecules involved in inflammation and is also involved in peroxisomal biogenesis (see also section 1.4.2; Itoyama et al. 2012; Braverman \& Moser 2012).

A prime suspect for triggering brain disease in peroxisomal $\beta$-oxidation deficiency is the accumulation of VLCFAs, which is also the major biochemical abnormality in human Xlinked Adrenoleukodystrophy, whose patients as well suffer from cerebral demyelination. VLCFA are incorporated into complex lipids such as gangliosides and phosphatidylcholine, and into branched chain FA, which are highly abundant in brain tissues. They are implied to enhance oxidative stress, possibly destabilize membranes, and lately have also been suggested to exert a negative effect on autophagy (Hein et al. 2008; Baarine, Ragot, et al. 2012; Baarine, Andréoletti, et al. 2012; Launay et al. 2014). However, increased VLCFA levels are also found in unaffected patient tissue and in brains of ABCD1-deficient mice, which do not develop a cerebral pathology, indicating that VLCFA accumulation itself not necessarily results in pathology (ForssPetter et al. 1997). 
The accumulation of branched chain FA (BCFA), especially phytanic and pristanic acid, has detrimental effects on the central nervous system, which is known from another peroxisomal disease caused by impaired a-oxidation, called Refsum disease (Schönfeld \& Reiser 2016). Both, phytanic and pristanic acids, are also mildly elevated in MFP2-deficient animals. However, since BCFA-rich diets increased plasma concentrations, but did not aggravate brain lesions, only a minor role is attributed to BCFA in the pathology caused by MFP2-deficiency (Huyghe et al. 2006).

The critical role of peroxisomal $\beta$-oxidation described above led to the speculation, whether lack of this pathway itself is primary responsible for brain disease also in PBDs, i.e. when peroxisomal function is completely absent. Although, the pattern as well as the hallmarks of neurodegeneration were reminiscent of $\mathrm{Cnp}{ }^{\mathrm{Cre} / \mathrm{Wt} *} \mathrm{Pex}^{-/-}$the

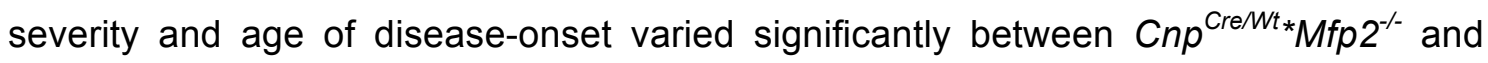

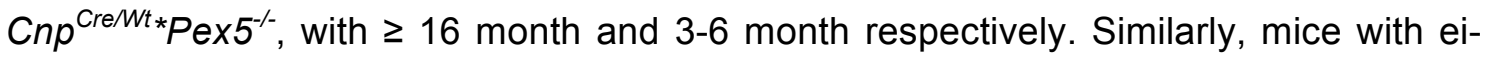
ther generalized or neural MFP2-deficiency, also show an alleviated phenotype compared to mice lacking peroxisomal function in the corresponding tissues completely (i.e.

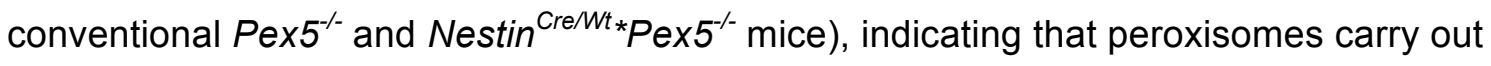
additional functions important for proper cell function (Baes et al. 1997; Hulshagen et al. 2008).

So far only mice without functional peroxisomes in myelinating glial cells $\left(C n p^{C r e / W t *} P e x 5^{-\alpha}\right)$ have been observed to exhibited callosal demyelination, a major hallmark of peroxisomal biogenesis defects and peroxisomal $\beta$-oxidation deficiency in humans. The here, analyzed $C n p^{C r e / W t *} M f p 2^{-/-}$animals is the first mouse model with a specific genetic impairment of peroxisomal $\beta$-oxidation that develops a subcortical demyelination reminiscent of the human pathology. This mouse model enables to investigate the possible effect of impaired peroxisomal $\beta$-oxidation on other peroxisomal metabolic pathways, on peroxisomal biogenesis, and on demyelination. Thus, it may also allow to study the prevention of secondary peroxisomal defects in the future, that may help preventing or delaying disease. 


\subsection{Cnp-mEos2-PTS1 mice enable cell type-specific analysis of peroxisomes}

The generation of the Cnp-mEos2-PTS1 transgenic mouse facilitates oligodendrocyte (OL) specific analysis of peroxisomes. The choice of a photoconvertible fluorescent protein significantly broadens the spectrum of applications, as it enables pulse-chase approaches that allow the investigation of peroxisomal biogenesis and peroxisomal degradation. The tripeptide peroxisomal targeting sequence, SKL (serine-lysineleucine), efficiently and specifically mediates peroxisomal import of the transgenic protein evidenced by mEos2 signal in peroxisomes, but absence from other organelles.

The Cnp promoter is expressed starting around embryonic day 13 in mice and allows the detection of peroxisomes from postnatal day 1 (P1) on, enabling the investigation of peroxisomes during development (Gravel et al. 1998). At P7 fluorescent peroxisomal structures were detectable throughout many brain regions and at p14 the final expression pattern was established. A high efficiency of oligodendrocytic targeting was proven by colocalization studies with the oligodendrocyte lineage marker protein Olig2. In white matter structures, but also in oligodendrocytes of grey matter, approximately 60$80 \%$ of Olig2 positive cells were associated with perinuclear mEos2 positive peroxisomes. Olig2 is a marker protein not only for mature OL, but also for oligodendrocyte precursor cells (OPC), of which only about $50 \%$ express Cnp (Saab et al., in preparation). Thus it can be expected that virtually all mature OL express mEos2, as described for comparable studies of Cnp dependent transgene expression elsewhere (Belachew et al. 2001). Although Cnp is detectable in Schwann cells, its expression is rather weak, thus mEos2 expression levels may not suffice for proper analysis of peroxisomes in the peripheral nervous system. mEos2 positive puncta were absent from other brain cells than oligodendrocytes, but were detectable in cells of spleen and testis consistent with published CNP-promoter activity (Davidoff, Middendorff, Köfüncü, Müller, Ježek, \& Holstein 2002b). Up to now analysis of peroxisomes in a cell type specific manner required time consuming colocalization studies due to high abundance of peroxisomes in the brain. The mEos 2 mouse significantly facilitates analysis of oligodendroglial peroxisomes.

The intraperoxisomal environment had no effect on the spectral properties of the $\mathrm{mE}$ os2 fluorophore or its photoconvertibility. Exposure to high intensity $\approx 370 \mathrm{~nm}$ light resulted in bright red signal with residual green fluorescence. Red fluorescence was not detectable in unexposed tissue or cells. 
The conversion of the total peroxisomal population in a cell allows to monitor the rate of newly formed (i.e. 'only green') peroxisomes as well as the rate of peroxisomal degradation (i.e. loss of red-labeled peroxisomes) similar to 'pulse-chase' experiments. Analysis of such experiments in Cnp-mEos2-PTS1 transgenic oligodendrocytes showed the reappearance of peroxisomes carrying only green mEos2 fluorescence already at d1 after photoconversion, indicative of peroxisomal de novo formation. However, these results have to be interpreted with caution as asymmetrical or subsequent fission events might also result in loss of photoconverted signal by dilution. So far no experimental approaches have been published that would allow a clear differentiation of de novo synthesis and fission under physiological conditions. Single cell analysis in CTRs revealed that about $40 \%$ of OLs showed an increase of photoconverted peroxisomes, proving that fission events can be observed in our experimental setup. Interestingly, in both populations of cells, those that displayed and those that did not exhibit peroxisomal fission, the number of peroxisomes containing only green mEos2 fluorescence increased equally.

Furthermore, instead of the whole peroxisomal pool also only a subpopulation of peroxisomes in a cell can be photoconverted allowing to investigate the fate of single organelles. Also the import-rate of peroxisomal proteins can be investigated via measurement of the green fluorescence intensity. In combination with a pulse-label approach measurement of intraperoxisomal fluorescence allows to compare protein import in newly formed as well as in 'aged' organelles. 


\subsection{Progressive loss of oligodendroglial peroxisomes in

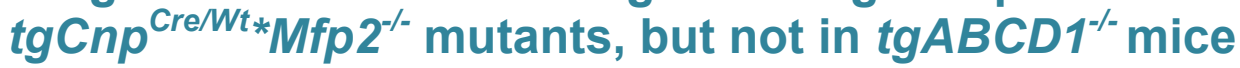

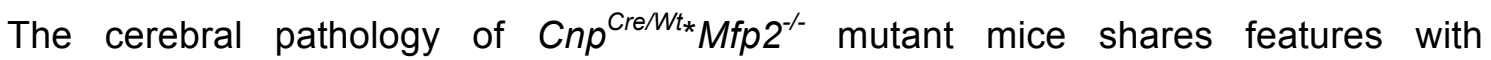
$\mathrm{Cnp}^{\mathrm{Cre} / \mathrm{Wt}_{*}} \mathrm{Pex} 5^{-{ }_{-1}}$ mice. This means that peroxisomal $\beta$-oxidation or complete peroxisomal function cause a similar pathology when ablated in oligodendrocytes of mice. In combination with a later onset and slower disease progression in $\mathrm{Cnp}{ }^{\mathrm{Cre} / \mathrm{Wt}_{*}} \mathrm{Mfp}^{-/-} \mathrm{mu}-$ tants, this allows to speculate on a secondary peroxisomal defect caused by impairment of peroxisomal beta-oxidation, which may reflect a complete loss of peroxisomal function.

To analyze oligodendroglial peroxisomes in vivo we made use of the CNP-mEos2-SKL

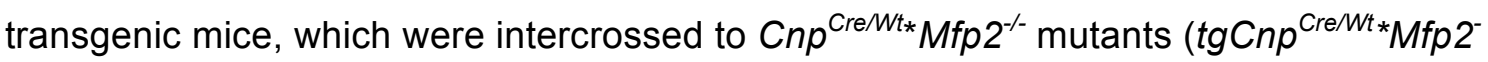
${ }^{1-}$ mice). $\operatorname{tgCnp}{ }^{\mathrm{Cre} /{ }^{*}{ }_{*}} M \mathrm{Mfp}^{-/-}$mutants revealed an early decrease of peroxisomes in the brain. Already at two months of age peroxisomal number per OL was reduced by $25 \%$ with a progressive loss to below $50 \%$ in $\leq 16$-month-old animals. Furthermore, the number of oligodendrocytes containing perinuclear mEos2 positive peroxisomes was decreased in old animals, culminating in a total loss of $2 / 3$ of peroxisomes per area. The reduced number of mEos2-expressing OL may be a result of reduced detection caused by the dramatic decrease of the number of mEos2 positive peroxisomes. Another possible explanation would be oligodendroglial cell death in the brains of aged mutants.

Interestingly, the reduction of the peroxisomal number was comparable between affected and unaffected brain white matter, suggesting that a decrease of peroxisomes is not directly resulting in demyelination, but via a yet unknown additional subcellular step of disease. Together our observations indicate that in $\operatorname{tgCnp}{ }^{\mathrm{Cre} / W t *}{ }^{*} \mathrm{Mfp}^{-/-}$mice, starting around two month of age, peroxisomal function, beyond that of peroxisomal $\beta$-oxidation, might be decreased and imply an explanation for the phenotypic similarities of $\mathrm{Cnp}{ }^{\mathrm{Cre} / \mathrm{Wt} *} \mathrm{Mfp}^{-/-}$to $\mathrm{Cnp}{ }^{\mathrm{Cre} / \mathrm{Wt} *} \mathrm{Pex5^{-/- }}$ mice.

Another interesting finding is the pronounced reduction of the number of 'peripheral peroxisomes' in $\operatorname{tgCnp}{ }^{\mathrm{Cre} / \mathrm{Wt} *}{ }^{*} \mathrm{Mfp} 2^{-/-}$mice, i.e. peroxisomes that are not closely associated with oligodendrocyte nuclei, but reside in the cellular periphery. These are supposedly located in cytoplasmic channels and other functional important sites, the paranodal loops of oligodendrocytes. In two-month-old animals peripheral peroxisomes were already reduced by half and in aged mutant only $20 \%$ were left. As this is also true for brain regions without overt demyelination, decrease in peripheral peroxisomes is likely 
not due to loss of oligodendroglial structures. Instead, the increased size of mutant peroxisomes might complicate the transport into the cellular periphery, or the reduced peroxisomal biogenesis might deregulate peroxisomal transport.

During the last 10 years the view of myelin serving only as a passive insulator has started to change to a more active role of myelin providing also metabolic support for axons (Fünfschilling et al. 2013). Peroxisomes, which are rarely found in axons, but are abundant in the different cytoplasmic compartments of myelin, possibly participate in axonal metabolic support (Kassmann et al. 2011). In this respect, the extreme loss of 'peripheral peroxisomes' in tgCnp ${ }^{\mathrm{Cre} / W t *} \mathrm{Mfp}^{-/-}$- mice, might be of particular importance for a possible function in axonal support. Recent studies of the peripheral nervous system of $\mathrm{Cnp}{ }^{\mathrm{Cre} / \mathrm{Wt} *} \mathrm{Pex} 5^{-/}$mice showed decreased axonal conduction velocity and axonal swellings in the absence of demyelination (Kassmann et al. 2011; Kleinecke et al., in preparation). This supports the hypothesis that possibly also oligodendroglial peroxisomes are important for such axo-glial interactions. Furthermore it raises the question whether additionally to demyelination an axonal problem might add to the pathology in $\operatorname{tg} \mathrm{Cnp}{ }^{\mathrm{Cre} / \mathrm{Wt} \star} \mathrm{Mfp}^{-/-}$.

cALD patients share the key features of inflammatory demyelination with patients of PBDs and other peroxisomal $\beta$-oxidation defects. However, the brains of aged $\operatorname{tg} A b c d 1^{-/}$mutants neither displayed a reduction of total, nor of peripheral peroxisomes. Also organelle morphology was apparently normal. Human skin fibroblast derived from $A B C D 1-d e f i c i e n t$ patients also contain normal peroxisomal numbers in contrast to MFP2-deficient patient's cells (Chang et al. 1999). This observed normal appearance of the peroxisomal population may be interpreted such, that the ABCD $1^{-{ }^{-}}$-related disease mechanism does not include a secondary failure of peroxisomal function as seen in MFP2-mutant oligodendrocytes. A second possible explanation would be, that even aged $A B C D 1^{-1-}$ mice are not suitable to model the subcellular disease mechanism, since this mouse model also fails to show demyelination even at very old age ( $>22$ months). Therefore, it would still be of interest to study peroxisomal morphology, especially of oligodendrocytes, in demyelinating lesions obtained from cALD patients to investigate, whether peroxisomal morphology is altered in a cell type specific manner. It is noteworthy, that such cell type-specific analysis is extremely difficult due to the high density of cells in myelin, and is further complicated by the invasion of astrocytes and microglia containing vast amounts of peroxisomes. 


\subsection{MFP2-deficiency decreases peroxisomal turnover in oli- godendrocytes}

Human skin fibroblasts derived from patients with peroxisomal biogenesis defects show a dramatic decrease of peroxisome numbers along with an increase in peroxisomal size. The same holds true for cells with impaired peroxisomal $\beta$-oxidation, but not for defective $\alpha$-oxidation or plasmalogen synthesis. Accumulating evidence suggests a role for peroxisomal $\beta$-oxidation in the control of organelle abundance. Another hint pointing to this direction is that overexpression of PTE1, an acyl-CoA hydrolase, which is thought to impair peroxisomal $\beta$-oxidation, shows a similar pattern of peroxisome reduction. The mechanism by which peroxisomal $\beta$-oxidation regulates peroxisomal abundance remains elusive so far (Chang et al. 1999).

Our analysis confirmed a reduction of peroxisomal number also in mEos2-SKL transgenic mice lacking MFP2-function from oligodendrocytes. In vitro 'pulse-chase' experiments using primary oligodendrocytes (OLs) additionally revealed prolonged detectability of photoconverted peroxisomes implying decreased degradation of the organelles in

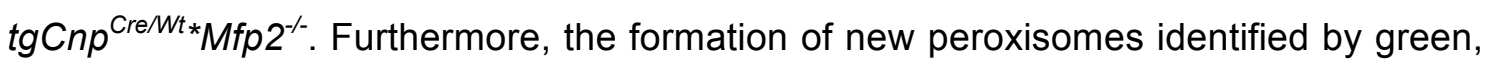
but lack of red fluorescence, accounted only for $\sim 17 \%$ of peroxisomes in mutant cells compared to $\sim 75 \%$ in control OLs.

Current publications imply a peroxisomal fission defect in PBDs and peroxisomal $\beta$-oxidation disorders (Itoyama et al. 2012; Bonekamp et al. 2013). We found that PEX11 $\beta$, which is essential for peroxisomal fission, is more abundant in myelin fractions compared to total brain lysates indicating a high contribution of fission to the biogenesis of oligodendroglial peroxisomes (unpublished data). In this respect, oligodendroglial peroxisomes may be especially vulnerable to impairment of peroxisomal fission.

Although it cannot be clearly differentiated, whether peroxisomal $\beta$-oxidation deficiency affects rather peroxisomal de novo synthesis, peroxisomal fission, or both, we measured reduced peroxisomal biogenesis, which was accompanied by increased stability of the organelle. Decreased peroxisomal turnover results in enhanced organelle aging. Aged organelles likely accumulate functional impairments as could be shown by Ivashchenko and coworkers, who encountered altered intraperoxisomal redox balance when pexophagy was impaired by 3-methyladenine treatment (Ivashchenko et al. 2011). 


\subsubsection{Direct effects of beta-oxidation impairment on fission?}

Patients' fibroblasts with a generalized or $\beta$-oxidation-specific peroxisomal defect show a comparable phenotype with less abundant enlarged peroxisomes. The same applies for loss-of-function mutations in proteins of the peroxisomal fission machinery, like PEX11, which is essential for peroxisomal fission ( $\mathrm{Li}$ et al. 2002). Interestingly, although endogenous expression of PEX11 $\alpha$ and PEX11 $\beta$ was normal, the overexpression of PEX11 $\beta$ led to normalization of the peroxisomal appearance proving that peroxisome proliferation is in principal possible in mutant cells with impaired peroxisomal biogenesis (Chang et al. 1999).

Another interesting observation has been reported from COS-7 cells grown in lipid-free and serum-free medium, which share the features of reduced peroxisomal abundance and increased organelle size. When PEX11 $\beta$ was overexpressed in these cells peroxisomal elongation was induced, but rare constriction and no fission, indicating a role for peroxisome membrane lipid composition in PEX11 $\beta$ dependent peroxisomal proliferation (Bonekamp et al. 2013). Itoyama and colleagues discovered that treatment with polyunsaturated FA particularly docosahexaenoic (DHA) and eicosapentaenoic acid (EPA), restored normal peroxisome numbers and morphology in human skin fibroblast with impaired peroxisomal $\beta$-oxidation. DHA is a highly abundant polyunsaturated FA in the brain whose synthesis depends on the endoplasmic reticulum and peroxisomal $\beta$-oxidation. DHA levels are usually strongly reduced in peroxisomal biogenesis defects or peroxisomal $\beta$-oxidation deficiency. A link between DHA-levels and PEX11 $\beta$ was recently shown by enhanced PEX $11 \beta$ oligomerization after treatment with DHA, suggesting that polyunsaturated FAs interact with PEX11 $\beta$ to form functional complexes (Itoyama et al. 2012). It remains to be investigated, if reduced DHA levels are causative for the observed peroxisomal morphology in MFP2-deficient oligodendrocytes. However, in contrast to data obtained from patients, DHA levels were normal even in conventional MFP2-deficient mice, which was attributed to the DHA-containing chow (Huyghe et al. 2006).

Besides oligomerization, also phosphorylation has been implicated to regulate Pex11 $\beta$ function. In yeast Pex11ßp-phosphorylation was increased under proliferating conditions, while expression of a constitutively dephosphorylated Pex11ßp resulted in enlarged peroxisomes (Knoblach \& Rachubinski 2010; Joshi et al. 2012). For mammals no such effect of mutations in putative phosphorylation sites on the proliferative properties of PEX11 $\beta$ have been identified so far (Bonekamp et al. 2013). It would be of inter- 
est to investigate, whether altered peroxisomal $\beta$-oxidation possibly influences peroxisomal fission via altered phosphorylation states of PEX11 $\beta$.

Furthermore, depletion of the peroxisomal fission machinery in yeast not only prevented peroxisomal fission, but also affected pexophagy providing evidence for an immediate link between biogenesis and degradation of peroxisomes (Mao et al. 2014; Manivannan et al. 2014).

\subsubsection{Direct effects of beta-oxidation impairment on autophagy?}

Interestingly, inhibition of autophagy by 3-methyladenine (3-MA) treatment in $\operatorname{tgCnp}{ }^{\mathrm{Cre} / \mathrm{Wt} * M f p 2^{-/-}}$OL showed not only diminished degradation, but also massively decreased biogenesis of peroxisomes. This constitutes an additional finding, which suggests direct coupling of peroxisomal degradation and biogenesis. 3-MA acts on autophagy by impeding autophagosome formation via the inhibition of PI3K (Laplante \& Sabatini 2009). To our knowledge no effects of 3-methyladenine or its target PIK3 on peroxisomal biogenesis have been described. This raises the question whether abrogated peroxisomal $\beta$-oxidation possibly affects peroxisomal abundance indirectly via autophagy impairment, which in turn might decrease peroxisomal biogenesis. Interestingly, peroxisomal numbers remained stable in autophagy impaired OLs in contrast to experiments in $\mathrm{CHO}$ cells, where peroxisomal number and cell size were increased upon 3-MA treatment. Thus control of peroxisomal abundance might be distinct in different cell types and possibly depends on the cells' ability to increase their volume. In contrast to the similar experiments with $\mathrm{CHO}$ cells, mature $\mathrm{OL}$ did not show an increase in size upon 3-MA treatment. This may suggest that regulation of peroxisomal abundance might be related to peroxisomal density.

An estimated $70-80 \%$ of peroxisomal degradation occurs by macroautophagy, termed pexophagy, while the remaining $20-30 \%$ are supposedly degraded by 15 -Lipoxygenase and peroxisomal LON protease (Yokota 2003; Kikuchi et al. 2004; Iwata et al. 2006). PEX5, the redox sensitive shuttling importer for the majority of peroxisomal proteins appears to play a dual role in pexophagy ( $\mathrm{Ma}$ et al. 2013). The negative regulators of mTORC1, TSC1, 2, and ATM have been found to localize to peroxisomes in a PEX5 dependent manner. Additionally, ATM kinase phosphorylates PEX5, which triggers subsequent ubiquitination and mediates binding to an autophagy receptor protein, to induce selective autophagosome engulfment (Zhang et al. 2013; 2015). It has been 
shown that impairment of VLCFA $\beta$-oxidation leads to perturbation of autophagysignaling, i.e. enhanced mTORC1 activity and decreased LC3II levels, in X-ALD patients and the corresponding ABCD1-deficient mouse mutants (Launay et al. 2014). Treatment of human skin fibroblasts with excess C26:0, a substrate of peroxisomal $\beta$-oxidation, exhibited similar dysregulation of autophagic markers. Administration of a mTORC1 inhibitor to ABCD1-deficient mice normalized the molecular findings and alleviated the macroscopic phenotype (Launay et al. 2014).

Increased cellular oxidative stress is a hallmark of peroxisomal disease (Fourcade et al. 2015); it causes accumulation of the PEX5 receptor on the peroxisomal membrane and reduces peroxisomal protein import (Apanasets et al. 2013). In our model the increase of intraperoxisomal green fluorescence proves that PEX5 dependent import of

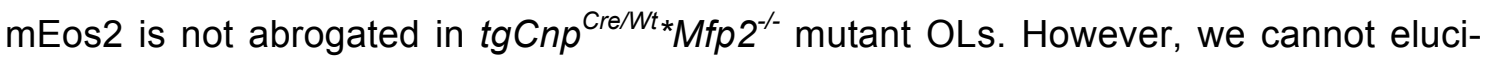
date whether it is fully functional. Impaired PEX5 function in cells lacking MFP2function might abrogate TSC2 signaling or impede selective autophagosome engulfment and cause decreased pexophagy. Co-precipitation of ATM-PEX5 or TSC2-PEX5 and immunohistochemical analysis of ATM kinase and TSC2 localization might give further insight.

Another mechanism for autophagy defects when $\beta$-oxidation is impaired might be directly linked to substrate-accumulation. In organisms with impaired peroxisomal $\beta$-oxidation peroxisomal substrates, e.g. VLCFA accumulate and might impair further cellular functions (Huyghe et al. 2006). VLCFA are frequently bound to other lipids, such as gangliosides and cholesterol, which require catabolic activity of other organelles (e.g. lysosomes) and possibly become non-degradable by a backlog mechanism. Accumulation of gangliosides bound to VLCFA associated with a secondary impairment of lysosomes was found in mutants lacking peroxisomal functions from Schwann cells (Cnp ${ }^{\mathrm{Cr} / \mathrm{Wt} *}$ Pex $5^{-/}$mutants; Kassmann et al. 2011; Kleinecke et al., publication in preparation). Similarly, tissue of patients suffering from the peroxisomal disease X-linked adrenoleukodystrophy (X-ALD), show increased VLCFA-bound gangliosides (Igarashi et al. 1976). Impaired degradation of peroxisomal substrates might thus result in an overall autophagy impairment, not only affecting pexophagy (Lieberman et al. 2012). A third possibility of autophagy impairment by accumulation of $\beta$-oxidation substrates is highlighted by data from Koga et al., which indicate that altered membrane lipid composition influences autophagosome lysosome fusion (Koga et al. 2010). 
Furthermore DHA was recently observed to induce autophagy via inhibition of the mTORC1 pathway. Decreased levels of polyunsaturated FAs in peroxisomal $\beta$-oxidation defects together with accumulation of VLCFA, which possibly exert a negative effect on autophagy, may thus affect normal autophagic flux and peroxisomal fission (Kim et al. 2015; Zhao et al. 2015).

Autophagy is crucial for proper brain function, as it prevents accumulation of cellular waste and dysfunctional organelles (Nixon 2013). Disrupted autophagy affects cell survival, increases oxidative stress and is likely involved in several neurodegenerative disorders like Alzheimer's and Parkinson's disease. Lysosomal storage diseases also show abolished autophagy (Lieberman 2012). Interestingly cerebral demyelination is a hallmark of lysosomal storage diseases raising the question whether impaired autophagy might be responsible for demyelination in peroxisomal diseases (Cheon et al. 2002). It remains to be investigated, whether perturbed autophagy-signaling or a simple backlog hitting lysosome function by non-degradable material are part of the pathomechanism also in $\mathrm{Cnp}^{\mathrm{Cre} / \mathrm{Wt} *} \mathrm{Mfp}^{-/-}$mutant mice.

The question is raised whether in MFP2-mutant cells a direct effect on peroxisomal biogenesis causes secondary problems in pexophagy, or vice versa inhibition of peroxisomal $\beta$-oxidation rather causes problems in pexophagy, which lead to secondary impairment of peroxisomal biogenesis. 


\subsection{Heterozygosity is not responsible for the pathology of Cnp ${ }^{\mathrm{Cre} / W{ }_{*}} \mathrm{Mfp}^{-/-}$mutants}

Heterozygous CNP gene dosage results in a late onset (> 24 month) mild axonal degeneration in mice (Hagemeyer et al. 2012). Furthermore Cre expression was related to increased oxidative stress and peroxisome proliferation in mice (Xiao et al. 2013). To exclude that the reduced Cnp gene dosage or the Cre expression itself cause the effects observed in $\operatorname{tgCnp}{ }^{\mathrm{Cre} /{ }^{*}{ }^{*} M f p 2^{-/}}$cells, Cnp-mEos2-SKL ${ }^{*} \mathrm{Cnp}{ }^{\mathrm{Cre} / W T}$ animals with normal MFP2 expression were analyzed. Pulse-label experiments in OL from these Cnp-heterozygous mice confirmed that peroxisomal alterations were neither attributable to Cre expression nor to Cnp heterozygosity. $\operatorname{tgCnp}{ }^{\mathrm{Cre} / W t} \mathrm{OL}$ did not exhibit decreased pexophagy as observed in $\operatorname{tgCnp}{ }^{\mathrm{Cre} / \mathrm{Wt} *} \mathrm{Mfp}^{-/}$. Instead, these peroxisomes showed more proliferation compared to cells with normal CNP- and without Cre expression during the first two days after photoconversion followed by rapid peroxisomal degradation. The here observed, enhanced proliferation in the early phase after photoconversion may be attributed enhanced Cre mediated stress (Xiao et al. 2013).

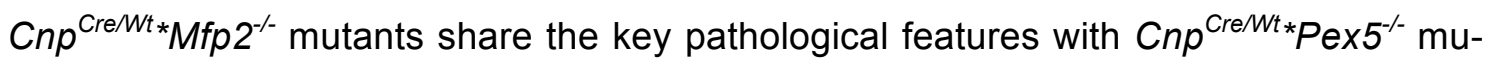
tant mice, which also have a reduced CNP-gene dosage in combination with Cre expression. Therefore, the finding of the much later disease onset and slower disease progression in MFP2-conditional mutants indicates that the pathology is indeed attributable to the depletion of peroxisomal function rather than to Cnp-heterozygosity or Cre expression. 


\section{Material}

\subsection{Antibodies}

Primary antibodies

$\begin{array}{ll}\text { ACAA1 } & \text { ProteinTech Group } \\ \text { APP } & \text { Chemicon } \\ \text { CD3 } & \text { Bio-Rad } \\ \text { GFAP } & \text { DAKO } \\ \text { Iba } & \text { Wako } \\ \text { Lamp1 } & \text { BD Pharmingen } \\ \text { Mac3 } & \text { BD Pharmingen } \\ \text { MBP } & \text { Dako } \\ \text { MRP } & \text { Antibodies-online } \\ \text { NeuN } & \text { Millipore } \\ \text { Olig2 } & \text { provided by J. Alberta Ligon et al., 2004 } \\ \text { PMP70 } & \text { Sigma-Aldrich } \\ \text { PMP70 } & \text { Abcam }\end{array}$

Secondary fluorescence-conjugated antibodies

Alexa-488

Invitrogen

Alexa-555

Invitrogen

DyLight 633

YO Proteins

\subsection{Bacterial strains}

E.coli XL1 blue bacteria

Statagene

\subsection{Enzymes}

SuperScriptlll Reverse Transcriptase

Invitrogen

GoTaq Polymerase

Promega

Red-Taq DNA Polymerase

Sigma-Aldrich

Pfu Ultra DNA Polymerase

Stratagene

Restriction enzymes

New England Biolabs

T4 DNA Ligase

Promega

DNase

Qiagen 


\subsection{Chemicals and Reagents}

Standard chemicals and reagents, which are not listed separately, were purchased from Sigma-Aldrich and Merck unless stated otherwise.

Agar

Agarose

Aqua Poly Mount

Aluminium sulfate

Bacto-Peptone

B27 Supplement

BSA

DAB

DAPI

DMEM

dNTP's $10 \mathrm{mM}$

FCS

GelRed

GeneRuler 1 kb ladder

GeneRuler 100 bp ladder

GlutaMax

HBSS

Horse Serum

Paraffin

Penicillin/Streptomycin

OptiMEM

Paraformaldehyde

Lipofectamin 2000 transfection reagent

Power SYBR Green PCR Master Mix

Protease-inhibitor tablets (Complete)

Sterile filters $(0.45 \mu \mathrm{m})$

TO-PRO-3

Trypsin/EDTA (0.25\%)
BD

BioFroxx

Polayscience Ltd.

Fluka

$\mathrm{BD}$

Thermo Fisher Scientific

Pan-Biotech

Dako Cytomation

Roche

Thermo Fisher Scientific

Roche

Thermo Fisher Scientific

Biotium

Fermentas

Fermentas

Thermo Fisher Scientific

Lonza

Thermo Fisher Scientific

Paraplast, Leica, Germany

Lonza

Thermo Fisher Scientific

Serva

Invitrogen

Applied Biosystems

Roche

Sartorius

Thermofisher

Thermo Fisher Scientific 
Trypsin

Yeast extract

\subsection{Kits}

nexttec $^{T m} 1^{- \text {Step }}$ DNA isolation Kit for Tissue \& Cells

NucleoSpin Plasmid QuickPure Kit

NucleoBond PC500 EF Maxi Kit

QIAquick Gel Extraction

RNeasy Mini Kit
Thermo Fisher Scientific

$\mathrm{BD}$

Invitek

Macherey-Nagel

Macherey-Nagel

Qiagen

Qiagen

\subsection{Laboratory supplies and equipment}

General laboratory equipment was purchased from Greiner Bio One, Falcon and Eppendorf.

Analytic scale AC $210 \mathrm{~S}$

Arium 611 Water Purification System

Automated system for Epon embedding

Biophotometer

Butterfly cannula $(27 G)$

Combi Thermosealer

Dispersing instrument Ultra-Turrax Polytron PT 3000

Gel documentation system

Incubator BB 6220

Laminar Flow hood Lamin Air HB 2448

MacMini

Paraffin Embedding Center

Peristaltic pump

$\mathrm{pH}-$ Meter

Pipetteboy

Pipettes

Sliding microtome HM400
Sartorius

Sartorius

EMPT Leica

Eppendorf

Venofix Nussloch, Germany

Advanced

Kinematica

Intas

Heraeus

Heraeus

Apple

MICROM HMP 110

Heraeus SR70

inoLab pH 720 WTW

Integra Science

Gilson

Mikrom 
Thermo mixer

Thermocycler T3000

UV-Illuminator UVT $40 \mathrm{~S} / \mathrm{L}$

Vibratome

Vortex Genie 2

Water bath

Micorscopes

Axio Observer Z1 Wide-field microscope

Axiophot Wide-field microscope

TCS SP5 Confocal microscope

\section{Centrifuges}

Table top centrifuge Biofuge Pico

Mikrocentrifuge

Ultrazentrifuge XL-70
Eppendorf

Biometra

Herolab

Leica VT 1000S, Leica

Bender\&Hobein AG

Memmert

Zeiss

Zeiss

Leica

Heraeus

Roth

Beckmann

\subsection{Oligonucleotides}

All oligonucleotides were synthesized at the 'DNA core facility' of the Max-PlanckInstitute of Experimental Medicine. They were provided at $50 \mathrm{pM}$ concentration.

\subsubsection{Genotyping}

CNP sense primer:

5'- GCCTTCAAACTGTCCATCTC-3'

CNP sense primer:

5'- CATAGCCTGAAGAACGAGA-3'

CNP antisense primer:

5' - CCCAGCCCTTTTATTACCAC-3'

Mfp2 sense primer:

5'-CCCAACGCTGGGTCACGGATGACGG-3'

Mfp2 antisense primer:

5'-GCAACCATAAGTTACACAAAATGCC-3'

Pxeos sense primer:

5' - CTTCTTACACAGGCCACCATGAGTGCG-3'

Pxeos antisense primer:

5'- GGATCCTTACTTAGTTAAAGCTTGGATCGT-3' 
$A B C D 1$ sense primer:

$A B C D 1$ antisense primer:

\subsubsection{Cloning}

Cnp1 sense primer:

Cnp1 antisense primer:

mEos2 sense primer:

mEos2 antisense primer:
5'-СССТTССТGCCАСТTTCATC-3'

5'-TCTCAACCTGCCССАССТCA-3

\subsection{Plasmids}

Cnp1-Cre

pGEM-T

pEGFP-N1

\subsection{Software}

Fiji

Illustrator CS3

Imaris

Lasergene

Office

OS X lion

SeqBuilder

Zen
Lappe-Siefke et al., 2003

Promega

Clontech

http://imagej.net/Fiji/

Adobe

Bitplane

DNASTAR

Windows

Apple

Lasergenes

Zeiss

\subsection{Molecular biology solutions}

\section{$50 \times$ Trisacetat EDTA (TAE) Buffer}

$2.0 \mathrm{M} \quad$ Tris-acetat, $\mathrm{pH} 8.0$

$50 \mathrm{mM} \quad$ EDTA

$57.1 \mathrm{ml} \quad$ glacial acid

fill up to $1 \mathrm{~L}$ with deionized $\mathrm{H}_{2} \mathrm{O}$ 
TE Buffer

LB Medium

LB Agar Plates
$10 \mathrm{mM}$

$1 \mathrm{mM}$

$\begin{array}{ll}0.5 \% & \text { yeast extract } \\ 1 \% & \text { Bacto-Peptone } \mathrm{pH} 7.5 \\ 1 \% & \mathrm{NaCl}\end{array}$

$0.5 \% \quad$ yeast extract

$1 \% \quad$ Bacto-Peptone $\mathrm{pH} 7.5$

$1 \% \quad \mathrm{NaCl}$

$1.5 \% \quad$ Agar

Autoclave solution and cool down to $55^{\circ} \mathrm{C}$ in a water bath before adding antibiotics.

\subsection{Histology buffers and solutions}

\begin{tabular}{|c|c|c|}
\hline \multirow[t]{3}{*}{ Avertin } & $2 \%$ & 2,2,2 Tribromethanol 99\% \\
\hline & $2 \%$ & Pentanol \\
\hline & & $\begin{array}{l}\text { Mixed at } 40^{\circ} \mathrm{C} \text { and stirred for } 30 \mathrm{~min} \text {, fil- } \\
\text { tered and stored at }-20^{\circ} \mathrm{C} \text {. }\end{array}$ \\
\hline \multirow[t]{2}{*}{$16 \%$ Paraformaldehyde } & $16 \%$ & Paraformaldehyde \\
\hline & & $\begin{array}{l}\text { Heated to } 65^{\circ} \mathrm{C} \text { and stirred for } 20 \text { min in } \\
\text { deionized } \mathrm{H}_{2} \mathrm{O} .5 \mathrm{M} \mathrm{NaOH} \text { droplets were } \\
\text { added until solution was cleared. The solu- } \\
\text { tion was then filtered and stored at }-20^{\circ} \mathrm{C} \\
\text { and thawed at } 60{ }^{\circ} \mathrm{C} \text { for } 30-45 \text { min before } \\
\text { usage. }\end{array}$ \\
\hline
\end{tabular}


4 \% Paraformaldehyde $\quad 10 \mathrm{ml}$

$40 \mathrm{ml}$

$25 \mathrm{ml}$

$25 \mathrm{ml}$
0.2M Sodiumdihydrogenphosphate

$\left(\mathrm{NaH}_{2} \mathrm{PO}_{4} \times \mathrm{H}_{2} \mathrm{O}\right)$

$0.2 \mathrm{M}$ di-Sodiumhydrogenphosphate

$\left(\mathrm{Na}_{2} \mathrm{HPO}_{4} \times \mathrm{H}_{2} \mathrm{O}\right)$

16\% Paraformaldehyde

deionized $\mathrm{H}_{2} \mathrm{O}$

\section{Bielschowsky Silver Impregnation}

\section{Solution A:}

Solution B:

Solution C:

Fixing solution:
$2.5 \mathrm{~g}$

$1 \%$

I. $2.5 \%$

II. $65 \mathrm{ml}$

III. $13 \mathrm{ml}$

$11 \mathrm{ml}$
Silver nitrate

Pyridine

in $250 \mathrm{ml}$ deionized $\mathrm{H}_{2} \mathrm{O}$

Silver nitrate solution: $2.75 \mathrm{~g}$ Silver nitrate in $110 \mathrm{ml}$ deionized $\mathrm{H}_{2} \mathrm{O}$

$96 \%$ Ethanol

$2.5 \% \mathrm{NaOH}$

concentrated $\mathrm{NH}_{3}$-Solution (32\% Merck)

The solutions (I-III) were joined sequentially. Solution III had to be added during constant stirring until the temporary brown participate cleared.
$0.675 \mathrm{ml}$
$40 \%$ formaldehyde
$0.03 \mathrm{~g}$
Citric acid (1\%)
$30 \mathrm{ml}$
Ethanol
in $250 \mathrm{ml}$ deionized $\mathrm{H}_{2} \mathrm{O}$

Sodium thiosulfate 


\section{Gallyas Silver Impregnation}

\begin{tabular}{|c|c|c|}
\hline \multirow[t]{5}{*}{ Incubation solution } & $0.1 \%$ & Ammonium nitrate \\
\hline & $0.1 \%$ & Silver nitrate \\
\hline & $12 \%$ & Sodium hydroxide $(\mathrm{pH} 7.5)$ \\
\hline & & in deionized $\mathrm{H}_{2} \mathrm{O}$ \\
\hline & & $\begin{array}{l}\text { The brown participate was dissolved by } \\
\text { shaking. The solution was stored up to } 8 \\
\text { weeks. }\end{array}$ \\
\hline Solution A: & $5 \%$ & $\begin{array}{l}\text { Sodium carbonate (dehydrated) in } \\
\text { deionized } \mathrm{H}_{2} \mathrm{O}\end{array}$ \\
\hline \multirow[t]{4}{*}{ Solution B: } & $0.2 \%$ & Ammonium nitrate \\
\hline & $0.2 \%$ & Silver nitrate \\
\hline & $1 \%$ & Wolframosilicic acid (silicotungstic acid) \\
\hline & & in deionized $\mathrm{H}_{2} \mathrm{O}$ \\
\hline \multirow[t]{6}{*}{ Solution C: } & $0.2 \%$ & Ammonium nitrate \\
\hline & $0.2 \%$ & Silver nitrate \\
\hline & $1 \%$ & Wolframosilicic acid (silicotungstic acid) \\
\hline & $0.26 \%$ & Paraformaldehyde \\
\hline & & in deionized $\mathrm{H}_{2} \mathrm{O}$ \\
\hline & & $\begin{array}{l}\text { The physical developer was prepared } \\
\text { freshly from solutions } A, B \text { and } C \text { in a } \\
10: 7: 3 \text { ratio. }\end{array}$ \\
\hline
\end{tabular}

Fixing solution:

$2 \%$

Sodium thiosulfate in deionized $\mathrm{H}_{2} \mathrm{O}$ 


\section{Haematoxylin-Eosin staining}

Mayer's Haematoxylin solution

$\begin{array}{ll}1 \mathrm{~g} & \text { Haematoxylin } \\ 0.2 \mathrm{~g} & \text { Sodium iodate } \\ 50 \mathrm{~g} & \begin{array}{l}\text { Potassium aluminium sulphate } \\ \text { Dissolved in } 1000 \mathrm{ml} \text { deionized } \mathrm{H}_{2} \mathrm{O} \text { during } \\ \end{array} \\ & \text { Constant shaking } \\ 50 \mathrm{~g} & \text { Chloral hydrate } \\ 1 \mathrm{~g} & \text { Citric acid }\end{array}$

\section{Eosin Solution}

Scott's solution

HCL-alcohol

$\begin{array}{ll}10 \mathrm{~g} & \text { Eosin } \\ 100 \mathrm{ml} & \text { deionized } \mathrm{H}_{2} \mathrm{O} \\ 2.5 \mathrm{ml} & \text { Stock solution } \\ 250 \mathrm{ml} & \text { deionized } \mathrm{H}_{2} \mathrm{O} \\ 12 \mathrm{drops} & \text { glacial acetic acid }\end{array}$

$2 \mathrm{~g} \quad \mathrm{KHCO}_{3}$

$20 \mathrm{~g} \quad \mathrm{MgSO}_{4}$

in $1 \mathrm{~L}$ deionized $\mathrm{H}_{2} \mathrm{O}$

$1.25 \mathrm{ml} \quad \mathrm{HCL}$

$350 \mathrm{ml} \quad$ Ethanol

$150 \mathrm{ml}$ deionized $\mathrm{H}_{2} \mathrm{O}$

\subsubsection{Chromogenic Immunohistochemistry}

\section{Citrate buffer}

Stock solution $A$ :

$0.1 \mathrm{M} \quad$ Citric acid

Stock solution B:

$0.1 \mathrm{M}$

Sodium citrate dehydrate

Stored at $4^{\circ} \mathrm{C}$

Working solution

$\begin{array}{ll}9 \mathrm{ml} & \text { Stock solution A } \\ 41 \mathrm{ml} & \text { Stock solution B } \\ 500 \mathrm{ml} & \text { deionized } \mathrm{H}_{2} \mathrm{O}\end{array}$

Always freshly prepared 


\section{Tris buffer}

Stock solution:

$0.5 \mathrm{M}$

Tris base

Adjusted to $\mathrm{pH} 7.6$ with $\mathrm{HCL}$

stored at $4^{\circ} \mathrm{C}$

Working solution:

$100 \mathrm{ml} \quad 0.5 \mathrm{M}$ Tris base

$9 \mathrm{~g}$

$\mathrm{NaCl}$

in $1000 \mathrm{ml}$ deionized $\mathrm{H}_{2} \mathrm{O}$

Always freshly prepared

Washing buffer

$2 \%$

milk powder

In Tris buffer

\section{Bovine Serum Albumin in PBS (PBS/BSA)}

$\begin{array}{ll}20 \mathrm{ml} & 0.2 \mathrm{M} \text { Sodiumdihydrogenphosphate } \\ 20 \mathrm{ml} & 0.2 \mathrm{M} \text { di-Sodiumhydrogenphosphate } \\ 1.8 \mathrm{~g} & \mathrm{NaCl} \\ 1 \mathrm{~g} & \mathrm{BSA} \\ 100 \mathrm{ml} & \text { deionized } \mathrm{H}_{2} \mathrm{O}\end{array}$

Blocking buffer $\quad 20 \% \quad$ Goat serum in PBS/BSA

\section{Luxol- fast- blue staining}

Nuclear fast red solution

$0.2 \mathrm{~g} \quad$ Nuclear fast red

$200 \mathrm{ml} \quad 5 \%$ Aluminium sulfate

Nuclear fast red was added to boiling aluminium sulfate and solution was further boiled for another 5-10 min thereafter. After cooling the solution was filtered immediately and again before each application.

Staining solution

$\begin{array}{ll}1 \mathrm{~g} & \text { Luxol blue } \\ 1000 \mathrm{ml} & 96 \% \text { ethanol }\end{array}$


Ethanol was stirred and warmed while Luxol blue was added. After cooling solution was filtered. Before use $50 \mu \mathrm{l}$ concentrated acetic acid is added per $100 \mu$ l staining solution

\subsubsection{Fluorescent immunohistochemistry}

$\begin{array}{lll}\text { Permeabilization solution } & 0.4 \% & \text { Triton X-100 in PBS } \\ \text { Blocking buffer } & 4 \% & \text { Horse serum } \\ & 0.2 \% & \begin{array}{l}\text { Triton X-100 } \\ \text { in PBS }\end{array} \\ & & \text { Horse serum } \\ 1^{\text {st }} \text { Antibody solution } & 1 \% & \text { Triton X-100 } \\ & 0.05 \% & \text { in PBS } \\ & & \text { Horse serum } \\ 2^{\text {nd }} \text { Antibody solution } 1 \% & 1.5 \% & \text { in PBS }\end{array}$

\subsection{Immunocytochemistry}

$10 \times$ PBS

$\begin{array}{ll}1.7 \mathrm{M} & \mathrm{NaCl} \\ 34 \mathrm{mM} & \mathrm{KCl} \\ 40 \mathrm{mM} & \mathrm{Na}_{2} \mathrm{HPO}_{4} \times 2 \mathrm{H}_{2} \mathrm{O} \\ 18 \mathrm{mM} & \mathrm{K}_{2} \mathrm{HPO}_{4}\end{array}$

in deionized $\mathrm{H}_{2} \mathrm{O}$; $\mathrm{pH} 7.2$ with $1 \mathrm{M} \mathrm{NaOH}$

Permeabilization solution

Blocking buffer

$1^{\text {st }}$ Antibody solution

$2^{\text {nd }}$ Antibody solution $1 \%$
$0.1 \%$ Triton $\mathrm{X}-100$ in PBS

$4 \% \quad$ Horse serum in PBS

$2 \% \quad$ Horse serum in PBS

$1.5 \%$ Horse serum

1:20000 DAPI or 1:5000 TO-PRO-3 in PBS 


\section{Electron Microscopy}

Epon

$\begin{array}{ll}171.3 \mathrm{~g} & \text { Glycidether } 100 \\ 115 \mathrm{~g} & \text { Dodecenyl succinic anhydride } \\ 89 \mathrm{~g} & \text { Methyl nadic anhydride } \\ & \text { Components were mixed using a } \\ & \text { magnet stirrer for } 10 \text { min and before } \\ & \text { adding } 6.5 \mathrm{ml} \text { DMP-30 and mixing } \\ & \text { for another } 20 \mathrm{~min} . \text { Prepare } 30 \text { min } \\ & \text { before embedding. }\end{array}$

\subsection{Solutions and media for primary oligodendrocytes}

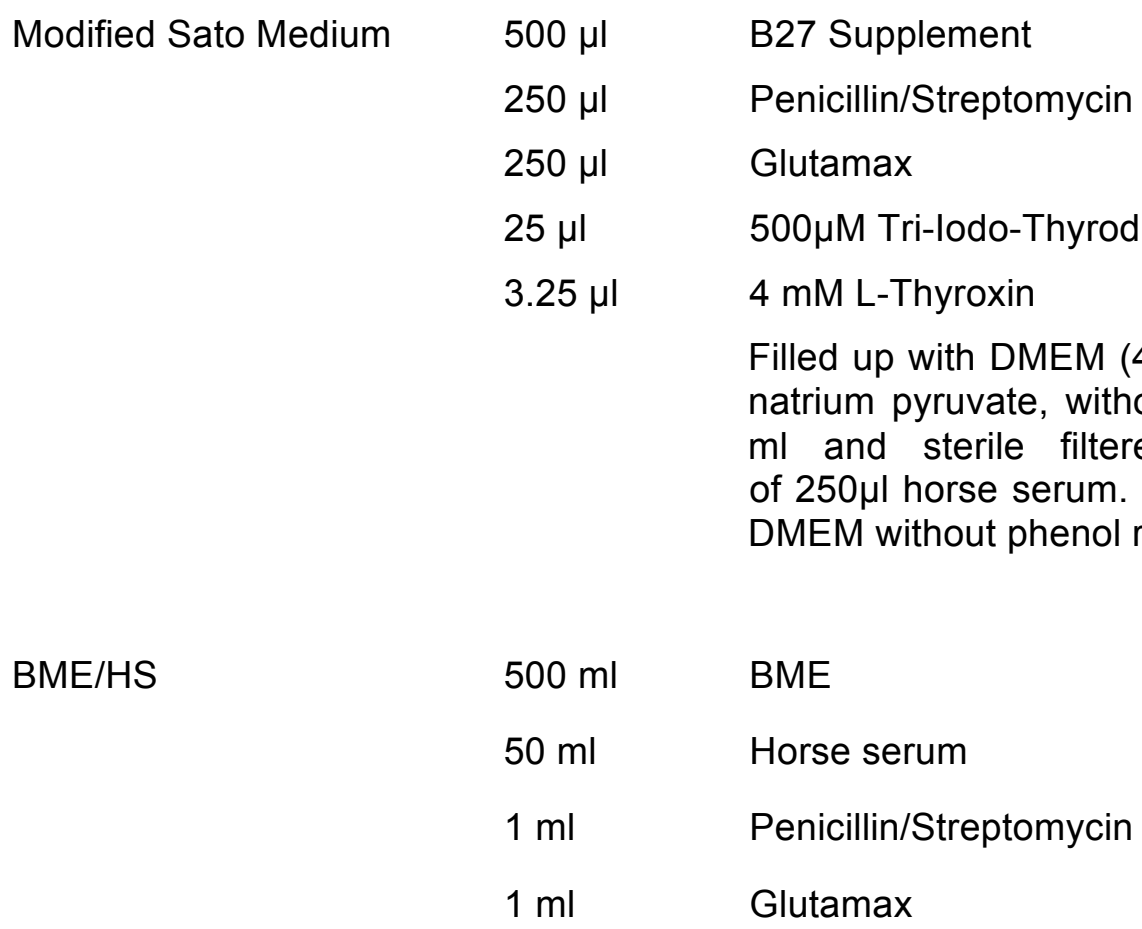

Poly-L-Lysine $\quad 0.1 \mathrm{mg} / \mathrm{ml} \quad$ Poly-L-Lysine in deionized $\mathrm{H}_{2} \mathrm{O}$

\subsection{Solutions for genomic DNA preparation}

$\begin{array}{lll} & 265 \mu \mathrm{l} & \text { Buffer G1 } \\ 10 \mu \mathrm{l} & \text { Buffer G2 } \\ 25 \mu \mathrm{l} & \text { Buffer G3 }\end{array}$




\section{Methods}

\subsection{Molecular biology methods}

\subsubsection{DNA isolation and purification}

\section{Genomic DNA preparation from mouse tissue}

Genomic DNA was purified with the 'nexttec ${ }^{T m} 1^{- \text {Step }}$ DNA isolation Kit for Tissue \& Cells'. Mouse tail biopsies were digested in $300 \mu$ lysis buffer while shaking with 1050 rpm at $62^{\circ} \mathrm{C}$ for $75 \mathrm{~min}$. After equilibration of the columns with $350 \mu \mathrm{l}$ Prep buffer and centrifugation, columns were loaded with $120 \mu$ tail digest and centrifuged again. The eluate was then diluted with $800 \mu$ ldeionized $\mathrm{H}_{2} \mathrm{O}$.

\section{DNA purification}

Macherey-Nagel's 'NucleoSpin Plasmid QuickPure Kit' and 'NucleoBond PC500 EF Maxi Kit' were used for small scale and large scale plasmid DNA purification, respectively. The method is based on a modified protocol by Birnboim and Doly (Birnboim and Doly, 1979). Cells undergo alkaline cell lysis followed by SDS precipitation subsequently plasmid DNA is bound to an anion exchange resin under appropriate $\mathrm{pH}$ and high salt conditions and eluted after washing.

Plasmid DNA was isolated from bacterial cultures grown in LB medium ON to an OD of 2 to 3 . For small scale purification $2-4 \mathrm{ml}$ of the bacterial culture were pelleted and resuspended in $250 \mu \mathrm{l}$ A1 buffer. Cell lysis was performed by addition of $250 \mu \mathrm{l}$ A2 buffer for $5 \mathrm{~min}$ followed by neutralization by addition of $A 3$ buffer. Subsequently genomic DNA and proteins were pelleted by centrifugation and the supernatant was loaded onto a column. The silica membrane was washed with $450 \mu \mathrm{l}$ AQ buffer, dried by centrifugation and plasmid DNA finally eluted in $100 \mu$ deionized $\mathrm{H}_{2} \mathrm{O}$.

For large scale plasmid purification $100 \mathrm{ml}$ of bacterial culture were pelleted and resuspended in $4 \mathrm{ml} \mathrm{S} 1$ buffer. Cell lysis was performed by addition of $4 \mathrm{ml} \mathrm{A2}$ buffer for 2-3 min followed by neutralization by $4 \mathrm{ml}$ precooled $\mathrm{S} 3$ buffer. After incubation on ice the solution was filtered before it was loaded onto the column that previously had been equilibrated with N2 buffer. After washing with $10 \mathrm{ml} \mathrm{N} 3$ buffer the DNA was eluted in 5 $\mathrm{ml} \mathrm{N5}$ buffer and precipitated using isopropanol. The pellet was resuspended in $100 \mu \mathrm{l}$ TE buffer. 


\subsubsection{DNA analysis and modification}

\section{Amplification of DNA by polymerase chain reaction}

Polymerase chain reaction was developed by Kay Mullis (Mullis et al., 1986) and enables in vitro amplification of DNA. The amplification begins with the annealing of short complementary oligonucleotides, so-called primers, to the sense and antisense strands of the DNA defining the template region. The thermostable DNA polymerase is able to bind ends of double strand DNA (primer and template) and elongate the complementary strand from the $5^{\prime}$ to the $3^{\prime}$ end. The first thermostable polymerase was discovered in Thermus aquaticus (Taq polymerase) and is widely used since. As it is a nonproofreading polymerase it introduces about 0.8 mismatches per $1 \mathrm{~kb}$ amplification cycle. By using proofreading polymerases the mismatch rate can be reduced.

PCR reactions consist of 3 main steps with multiple repetitions that yield an exponential increase of the product. Primarily DNA is denaturated at $95^{\circ} \mathrm{C}$ followed by primer annealing at a temperature that depends on the length and composition of the primer. Subsequently elongation takes place at around $72^{\circ} \mathrm{C}$. The time required for elongation depends on the length of the template as well as the polymerase used. Nonproofreading polymerases require about $30 \mathrm{sec}$ per $1 \mathrm{~kb}$ template where as proofreading poylmerases require up to $1 \mathrm{~min}$ per $1 \mathrm{~kb}$.

\section{Standard PCR reaction}

$\begin{array}{lllll}\text { Template }(10-100 \mathrm{ng}) & \mathrm{X} \mu \mathrm{l} & \text { 1. Initial denaturation } & 3 \mathrm{~min} & 95^{\circ} \mathrm{C} \\ \text { Sense primer }(10 \mathrm{pmol} / \mu \mathrm{l}) & 1 \mu \mathrm{l} & \text { 2. Annealing } & 30 \mathrm{sec} & 56^{\circ} \mathrm{C} \\ \text { Antisense primer }(10 \mathrm{pmol} / \mu \mathrm{l}) & 1 \mu \mathrm{l} & \text { 3. Elongation } & 1 \mathrm{~min} & 72^{\circ} \mathrm{C} \\ 10 \times \text { reaction buffer } & 2 \mu \mathrm{l} & \text { 4. Denaturation } & 30 \mathrm{sec} & 95^{\circ} \mathrm{C} \\ \text { dNTP mix }(0.2 \mathrm{mM} \mathrm{each}) & 5 \mu \mathrm{l} & \text { Repetition of } 2-435 \mathrm{x} & & \\ \text { Taq Polymerase }(5 \mathrm{U} / \mu \mathrm{l}) & 0.8 \mu \mathrm{l} & \text { 5. Final annealing } & 1 \mathrm{~min} & 55^{\circ} \mathrm{C} \\ \text { Add deionized } \mathrm{H}_{2} \mathrm{O} \text { to } 20 \mu \mathrm{l} & & \text { 6. Final elongation } & 10 \mathrm{~min} & 72^{\circ} \mathrm{C}\end{array}$




\section{Agarose Gel electrophoresis}

DNA molecules are negatively charged resulting in migration towards positively charged electrodes allowing size dependent separation. For the size separation of DNA fragments agarose gels with concentrations of $1-2 \%$ were used. Agarose was diluted in $1 \mathrm{x}$ TAE and heated in a microwave until the agarose was completely dissolved. The solution was poured into gel casting chambers and combs inserted to define pockets for sample loading. After cooling the gel was transferred to an electrophoresis chamber and covered with $1 \times$ TAE buffer. Samples mixed 1:5 with pre-diluted GelRed (1:4000) were loaded next to appropriate DNA markers (100 bp or $1000 \mathrm{bp}$ ladder). The voltage applied depended on the agarose concentration used. The separated DNA fragments were visualized by UV light illumination.

\section{Photometric concentration determination of nuclear acids}

According to the Lambert-Beer law the concentration of an aqueous solution is proportional to its absorbance and thus can be measured by spectrophotometry. Different groups of molecules have individual absorption maxima. Absorbance at $260\left(A_{260}\right)$ corresponds to DNA, at $280 \mathrm{~nm}\left(\mathrm{~A}_{280}\right)$ to protein and at $230 \mathrm{~nm}\left(\mathrm{~A}_{230}\right)$ to phenol and guanidium whereas absorbance at $320 \mathrm{~nm}$ accounts for the turbidity of the sample and is generally subtracted from the $A_{260}$ value. This allows the measurement of DNA/RNA concentration as well as contaminations.

For measurement DNA was diluted $1: 50$ in deionized $\mathrm{H}_{2} \mathrm{O}$ in a $100 \mu \mathrm{l}$ volume in a disposable plastic cuvette and measured at the above mentioned wavelengths. For a reliable measurement the $A_{260}$ value must lie between 0.1 and 1 , the linear range of the photometer. Sample purity was estimated from the ratios $A_{260} / A_{280}$ and $A_{260} / A_{230}$. Good quality samples have a $A_{260} / A_{280}$ ratio of 1.7-2.0 and an $A_{260} / A_{230}$ ratio of greater than 1.5 .

\section{Restriction digest}

Restriction endonucleases derived from bacteria or archea recognize and cleave specific, mostly palindromic DNA sequences leaving 5' or 3'overhangs, so-called sticky ends, or blunt ends depending on the enzyme. This technique enables ligation of DNA strands with compatible ends (preparative digest). Additionally endonuclease restriction with two or more endonucleases yields a specific fragment pattern helping to identify DNA (analytic digest). In a 20 or $25 \mu$ l volume, $500 \mathrm{ng}$ to $5 \mu \mathrm{g}$ of DNA were digested by $1-10 \mathrm{U}$ of enzyme at $37^{\circ} \mathrm{C}$ for $1 \mathrm{~h}$. Most enzymes can be heat-inactivated at $65^{\circ} \mathrm{C}$ for $20 \mathrm{~min}$. 


\section{Agarose Gel DNA extraction}

Agarose gel DNA extraction was performed with the kit 'QIAquick Gel Extraction' provided by Qiagen. After DNA separation by gel electrophoresis the desired DNA fragment was excised under UV light illumination. The amount of QG buffer added depended on the weight of the excised agarose. The mix was incubated at $50^{\circ} \mathrm{C}$ for 10 min until the agarose was completely dissolved. The solution was loaded onto a silica membrane washed with $750 \mu \mathrm{l} \mathrm{PE}$ buffer and eluted in $30 \mu \mathrm{l}$ EB buffer.

\section{Ligation}

The formation of phosphodiester bonds between 3'hydroxyl and 5'phosphoryl groups of DNA with compatible ends is called ligation and can be catalyzed by a DNA ligase derived from the bacteriophage T4. Ligation was carried out in volume of $10 \mu$ l containing $3 \cup \mathrm{T} 4$ ligase in buffer with vector and insert DNA in a molar ratio of 1:3. The reaction incubated for $1 \mathrm{~h}$ at RT and was stopped at $65^{\circ} \mathrm{C}$ for $20 \mathrm{~min}$.

\section{Ligation pGEM-T Vector}

The commercially available pGEM-T vector is a linearized vector with 3'-terminal thymidine overhangs allowing easy ligation with inserts flanked by adenine overhangs, so called TA cloning. Additionally pGEM-T contains a lacZ gene with an integrated multiple cloning site as well as ampicillin resistance gene. To prepare inserts for cloning into the pGEM-T vector a single additional PCR step is included with the Red-Taq polymerase. The Red-Taq polymerase adds a adenine to the 3 'ends of the insert rednering it compatible to the pGEM-T vector. Ligation is carried out as described in section 7.1.2.6.

\section{DNA sequencing}

DNA samples were sequenced at the 'DNA core facility' of the Max-Planck-Institute of experimental Medicine. $16 \mu \mathrm{l}$ DNA sample diluted down to $100 \mathrm{ng} / \mu \mathrm{l}$ in deionized $\mathrm{H}_{2} \mathrm{O}$ were submitted for sequencing. Preexisting sequencing primers were chosen or new primers designed and synthesized by 'DNA core facility'. Sequencing data was analyzed using the Lasergene EditSeq software.

\section{Primer design}

Primers were designed manually with Lasergenes SeqBuilder software and BLAST at NCBI. Primers had a 20 bp overlap with the template and a melting temperature of 55- 
$60^{\circ} \mathrm{C}$. The melting temperature was determined with the help of an online algorithm (http://www.basic.northwestern.edu/biotools/oligocalc.html). All primers were synthesized at the 'DNA core facility' of the Max Planck Institute of Experimental Medicine.

Intron spanning primers for qRT-PCR were designed using the online Universal ProbeLibrary Assay Design Center by Roche (https://lifescience.roche.com/webapp/wcs/stores/servlet/CategoryDisplay?tab=Assay+ Design+Center\&identifier=Universal+Probe+Library\&langld=-1)

\section{Cloning of the construct for generation of Cnp-mEos2-SKL transgenic mouse}

A plasmid containing the mEos2 complete coding sequence was amplified using two primers. The antisense primer contained a 9 bp sequence encoding Ser, Lys, Leu for peroxisomal targeting followed by a stop codon and a BamHI sequence. Several amplification cycles using pfu DNA polymerase were carried out in a thermo cycler and terminated with a single amplification cycle using red-taq DNA polymerase for the addition of 3 'adenine overhang for further ligation into the pGEM-T vector via TA cloning. The $687 \mathrm{bp}$ sequence containing mEos2-SKL and a stop codon was cloned into the Cnp1promoter construct via the AfllI and BamHI restriction sites. The final product contained $3.9 \mathrm{~kb}$, which included the Cnp promoter, $687 \mathrm{bp}$ that encompassed the mEos 2 coding sequence with the PTS1 and a SV40 PolyA sequence downstream consisting of 120 bp. The $5.1 \mathrm{~kb}$ sequence was cut out via the restriction enzymes $\mathrm{Xbal}$ and $\mathrm{BamHI}$ and purified for microinjection into C57BL/6N fertilized oocytes.

\subsubsection{Transformation of chemically competent bacteria}

Chemically competent E.coli XL1 blue bacteria were thawed on ice. A fraction of the ligation reaction was added to $100 \mu \mathrm{l}$ bacteria suspension and incubated for $30 \mathrm{~min}$ on ice followed by a heat shock in a water bath at $42^{\circ} \mathrm{C}$ for $40 \mathrm{sec}$. After another 2 min on ice $800 \mu \mathrm{LBB}$ media were added and the suspension was left to rotate at $37^{\circ} \mathrm{C}$. After 30 min varying amounts of solution were plated onto LB agar plates supplemented with the corresponding antibiotic. Colonies were left to grow at $37^{\circ} \mathrm{C}$ and single colonies were picked the next day to inoculated $4 \mathrm{ml}$ of antibiotic containing LB medium, which was incubated over night and used for a small scale DNA purification.

\subsection{Histological methods}

\subsubsection{Intracardial Perfusion}

Mice were anesthetized by intraperitoneal injection of Avertin (100 $\mu \mathrm{l} / 10 \mathrm{~g}$ bodyweight) and anesthetic depth was determined by the toe pinch-response method. After opening 
the rip cage and dissection of the heart a butterfly cannula (27G, Venofix) was inserted into the left ventricle and the right atrium was incised. The animal was perfused with 50 $\mathrm{ml} \mathrm{HBSS}$ followed by $40-50 \mathrm{ml} 4 \%$ PFA in phosphate buffer with the help of a peristaltic pump (Heraeus SR70, flow rate 0,2-0,5 $\mathrm{ml} / \mathrm{min}$ ). Brains were dissected and post fixed in the same fixative at $4^{\circ} \mathrm{C}$ for $4 \mathrm{~h}$ and then placed into $1 \%$ PFA ON until either vibratome sections were taken or further processing for paraffin embedding took place the following day. For epon embedding the tissue could be stored longer in $1 \%$ PFA.

\subsubsection{Procedures for tissue embedding and sectioning}

\section{Sectioning of PFA-fixed tissue}

Brains from intracardially perfused mice were post fixed over night in $1 \%$ PFA before 40-50 $\mu \mathrm{m}$ sections were generated with a vibratome (Leica VT 1000S, Leica Instruments, Nussloch, Germany). Slices were transferred to 24-well plates containing PBS.

\section{Paraffin embedding and sectioning}

Tissues fixed with 4\% PFA for at least $4 \mathrm{~h}$ were dehydrated in a Tissue Processor (MICROM HMP 110) by incubation in ethanol baths with an ascending ethanol concentration followed by clearing with xylene, which ultimately was replaced by paraffin. Paraffinated brains were embedded in $60^{\circ} \mathrm{C}$ paraffin in metal molds with a Paraffin Embedding Station (MICROM AP280) cooled down and stored at RT. Sections (5 $\mu \mathrm{m})$ were taken with a sliding microtome (MICROM HM400). For immunohistochemical stainings sections were deparaffinated and rehydrated by the reverse procedure as mentioned above.

\section{Dehydration and paraffination}

$\begin{array}{ll}\text { Procedure } & \text { Time } \\ 50 \% \text { Ethanol } & 1 \mathrm{~h} \\ 70 \% \text { Ethanol } & 2 \times 2 \mathrm{~h} \\ 96 \% \text { Ethanol } & 2 \times 2 \mathrm{~h} \\ 100 \% \text { Ethanol } & 2 \times 2 \mathrm{~h} \\ \text { Isopropanol } & 1 \mathrm{~h} \\ \text { Xylole } & 2 \times 2 \mathrm{~h}\end{array}$

\section{Deparaffination and rehydration}

$\begin{array}{ll}\text { Procedure } & \text { Time } \\ 60^{\circ} \mathrm{C} & 10 \mathrm{~min} \\ \text { Xylole } & 2 \times 10 \mathrm{~min} \\ \text { Xylole/isopropanol }(1: 1) & 10 \mathrm{~min} \\ 100 \% \text { Ethanol } & 5 \mathrm{~min} \\ 90 \% \text { Ethanol } & 5 \mathrm{~min} \\ 70 \% \text { Ethanol } & 5 \mathrm{~min} \\ 50 \% \text { Ethanol } & 5 \mathrm{~min} \\ \text { deionized } \mathrm{H}_{2} \mathrm{O} & 5 \mathrm{~min}\end{array}$




\section{Epon embedding and sectioning}

Tissue for electron microscopy imaging was osmified, dehydrated and epon embedded in an automated system (EM-TP, Leica). Epon was freshly prepared 30 min prior to embedding. After washing the fixed tissue with $0.1 \mathrm{M}$ phosphate buffer the material was placed into the embedding machine in plastic chambers and the following program was carried out:

$\begin{array}{lll}\text { Phosphate buffer } & 10 \mathrm{~min}, 3 \mathrm{x} & 4^{\circ} \mathrm{C} \\ 2 \% \mathrm{OsO}_{4} & 4 \mathrm{~h} & 4^{\circ} \mathrm{C} \\ \text { deionized } \mathrm{H}_{2} \mathrm{O} & 10 \mathrm{~min}, 3 \mathrm{x} & 4^{\circ} \mathrm{C} \\ 30 \% \text { Ethanol } & 20 \mathrm{~min} & 4^{\circ} \mathrm{C} \\ 50 \% \text { Ethanol } & 20 \mathrm{~min} & 4^{\circ} \mathrm{C} \\ 70 \% \text { Ethanol } & 20 \mathrm{~min} & 4^{\circ} \mathrm{C} \\ 90 \% \text { Ethanol } & 20 \mathrm{~min} & 4^{\circ} \mathrm{C} \\ 100 \% \text { Ethanol } & 20 \mathrm{~min}, 4 \mathrm{x} & 4^{\circ} \mathrm{C} \\ \text { Propylenoxide } & 10 \mathrm{~min}, 3 \mathrm{x} & \mathrm{RT} \\ \text { Propylenoxide /Epon } 2: 1 & 2 \mathrm{~h} & \mathrm{RT} \\ \text { Propylenoxide /Epon } 1: 1 & 2 \mathrm{~h} & \mathrm{RT}\end{array}$

Afterwards tissue was placed into epon filled molds and left to polymerize at $60^{\circ} \mathrm{C}$ over night. Epon embedded tissue was cut using a microtome (Ultracut $\mathrm{S}$, Leica) equipped with diamond knives (Diatome Hist $45^{\circ}$ and Ultra $45^{\circ} \mathrm{C}$ ) into ultrathin $(50 \mathrm{~nm})$ sections. Slices were placed on slot grids coated with Formvar polyvinyl and subsequently contrasted. Epon embedding and sectioning was performed by Wiebke Möbius and Boguslawa Sadowski.

\subsubsection{Staining procedures}

\section{Bielschowsky's Silver Impregnation}

For visualization of axonal loss neurons were visualized by Bielschowsky's Silver Impregnation, which exclusively stains neuronal cell bodies and axons. Deparaffinated and rehydrated paraffin sections were incubated in $1 \%$ silver nitrate (solution A) for 30 min at RT and dipped into ammoniacal silver nitrate solution for $10 \mathrm{sec}$ (solution B) before they were incubated in developing solution (solution $\mathrm{C}$ ) up to $5 \mathrm{sec}$ and rinsed with deionized $\mathrm{H}_{2} \mathrm{O}$. The staining was fixed by 5 min incubation in $2 \%$ sodium thiosulfate and sections were rinsed again in deionized $\mathrm{H}_{2} \mathrm{O}$ and dehydrated and mounted with Aqua polymout or Eukitt. 


\section{Gallyas Silver Impregnation}

Staining of myelinated fibers by colloidal silver was developed by Gallyas (Gallyas, 1979). Deparaffinated and rehydrated paraffin sections were incubated in a 2:1 solution of pyridine and acetic anhydride for $30 \mathrm{~min}$, washed three times in deionized $\mathrm{H}_{2} \mathrm{O}$ for $10 \mathrm{~min}$ each and left in warm incubation solution for $10 \mathrm{~min}$. Afterwards sections were washed with $0,5 \%$ acetic acid three times for $5 \mathrm{~min}$ and incubated in developer solution for 3-15 min until the staining was discernable under a light microscope. Color development was stopped by washing in $1 \%$ acetic acid followed by washing with deionized $\mathrm{H}_{2} \mathrm{O}$ and fixation of the staining with $2 \%$ sodium thiosulfate for $5 \mathrm{~min}$, dehydration and mounting with Aqua polymout or Eukitt.

\section{Chromogenic immunohistochemistry on paraffin sections}

For chromogenic immunohistochemical stainings the secondary antibody is coupled to horseradish peroxidase, which oxidizes its substrate 3,3'Diaminobenzidine (DAB) yielding a brown precipitate. Deparaffinated and rehydrated paraffin sections were incubated for $5 \mathrm{~min}$ in citrate buffer and then cooked in boiling citrate buffer for $10 \mathrm{~min}$ at $650 \mathrm{~W}$ in a microwave for antigen retrieval. After cooling, sections were washed for 5 min in Tris buffer with $2 \%$ milk powder and the slides containing sections were mounted in plastic cover plates in, which the following steps were carried out. $3 \%$ hydrogen peroxide was added for $5 \mathrm{~min}$ to inactivate endogenous peroxidases followed by blocking buffer for $20 \mathrm{~min}$. Subsequently the primary antibody solution in PBS/BSA was applied and incubated $\mathrm{ON}$ at $4^{\circ} \mathrm{C}$. After washing with $2 \%$ milk powder in Tris buffer the biotinylated secondary antibody (Dako LSAB2, solution A) was added for 10 min followed by another washing step in Tris buffer with $2 \%$ milk and incubation in streptavidin conjugated horseradish peroxidase solution (Dako LSAB2, solution B) for $10 \mathrm{~min}$. Washing with Tris buffer was performed again before the incubation with DAB substrate solution (1 $\mathrm{ml}$ Dako substrate buffer with two drops of DAB) for $10 \mathrm{~min}$. Finally a counterstaining with Haematoxylin was performed and the section were rehydrated and mounted with Aqua polymout or Eukitt.

\section{Haematoxylin-Eosin (HE) staining}

Haematoxylin staining colors nuclei dark purple while treament with Eosin results in a pink coloring of eosinophilic structures, such as cytoplasm and collagen. Combination of the dyes provides a detailed visualization of overall tissue structure. Deparaffinated and rehydrated paraffin sections were incubated in $0.1 \%$ Haematoxylin for $5 \mathrm{~min}$, washed with deionized $\mathrm{H}_{2} \mathrm{O}$ and excess dye was removed by a short dipping in HCLalcohol solution. For contrast enhancement sections were treated with Scott's solution for 5 min before being rinsed with deionized $\mathrm{H}_{2} \mathrm{O}$ and counterstained with $0.1 \%$ Eosin 
for 3-5 min. After washing sections were dehydrated and mounted with Aqua polymout or Eukitt.

\section{Fluorescent immunohistochemistry on fixed tissue}

Vibratome sections were permeabilized in $0.4 \%$ Triton X-100 in PBS for 30 minutes. Blocking was performed for 30 minutes in $4 \%$ Horse serum (HS) and $0.2 \%$ Triton $X-$ 100 in PBS. Primary antibody incubation was carried out overnight at $4^{\circ} \mathrm{C}$ in $1 \% \mathrm{HS}$ and $0.05 \%$ Triton X-100 in PBS followed by two washing steps in PBS for $5 \mathrm{~min}$. Incubation in the secondary antibodies solution was performed for 2 hours in $1.5 \% \mathrm{HS}$ in PBS containing either DAPI (1:20.000) or Topro (1:5000). Sections were washed twice in PBS and transferred to slides in tap water before mounting in Aqua Poly mount (Polysciences, Warrington, PA).

\section{Luxol- fast- blue staining}

Luxol- fast- blue stains CNS myelin in bright blue whereas neurons are stained violet allowing the analysis of myelinated structures as well as neuronal structures of the brain. Deparaffinated and rehydrated paraffin sections were incubated in $0.1 \%$ Luxolfast- blue staining solution at $56^{\circ} \mathrm{C} \mathrm{ON}$, flushed in $96 \%$ ethanol and washed in deionized $\mathrm{H}_{2} \mathrm{O}$ before incubation in $0.1 \%$ lithium carbonate for $5 \mathrm{~min}$. Sections were then dipped shortly into $70 \%$ ethanol, washed with deionized $\mathrm{H}_{2} \mathrm{O}$ and counterstained with nuclear fast red for 10 min. Finally sections were dehydrated and mounted.

\section{Tissue contrasting for electron microscopy}

Grids containing the epon slices were placed with the shiny side up onto the drops of the following solutions for contrasting and subsequently dried with filter paper.

$\begin{array}{ll}4 \% \text { Uranyl acetat } & 30 \mathrm{~min} \\ \text { deionized } \mathrm{H}_{2} \mathrm{O} & 1 \mathrm{~min}, 3 x \\ \text { Reynolds lead citrate } & 6 \mathrm{~min} \\ \text { deionized } \mathrm{H}_{2} \mathrm{O} & 1 \mathrm{~min}, 4 \mathrm{x}\end{array}$




\subsection{Cell biology methods}

Preparation of cell culture media and solutions was performed in the sterile environment of a laminar flow hood. Cell cultures were kept at $37^{\circ} \mathrm{C}$ in a humidified incubator with $5 \% \mathrm{CO}_{2}$.

\subsubsection{Preparation and culture of primary oligodendrocytes}

Brains from newborn mice (P0/P1) were prepared and transferred to a petri dish containing warm HBSS. The meninges were carefully removed under a binocular together with the olfactory bulbus, the cerebellum, and the midbrain. The tissue was transferred to $15 \mathrm{ml}$ Falcon tubes and $1 \mathrm{ml} 0.25 \%$ trypsin-EDTA in BME per brain was added for 5 min. The trypsin-EDTA was then removed and the brain washed twice with BME medium. Brains were repeatedly passed through Pasteur pipettes in a volume of $1 \mathrm{ml}$ BME/HS per brain for dissociation of the tissue. This procedure was carried out until a homogenous solution was achieved. $1 \mathrm{ml}$ of cell suspension was added to $14 \mathrm{ml}$ preheated BME/HS medium in cell culture flasks coated with Poly-L-Lysine and cultivated at $37^{\circ} \mathrm{C}$ and $5 \% \mathrm{CO}_{2}$. Three days later and then every second day medium was exchanged. About 7 days after plating a confluent astrocyte layer was formed, on which oligodendrocyte precursor cells (OPC) and microglia were growing. 10 days after, culturing microglia were removed by careful agitation of the flask and exchange of the BME/HS medium. OPC were harvested by 30 times vigorously shaking the cell cultures flasks and pelleting the cells by centrifugation of the supernatant. Cells were resuspended in Sato medium and counted in a Neubauer chamber. Finally 50000-70000 cells were plated in Sato medium in PLL coated 24-well plates and cultivated as described above. For live cell imaging DMEM without phenol red was used and cells plated on 24 well plates with \# 1.5 coverslips. Live cell experiments were conducted in mature primary oligodendrocytes 8 days after plating.

\subsubsection{Poly-L-Lysine coating}

Cell culture dishes, flasks and cover slips were coated with poly-L-lysine (PLL) for better adherence of cells mediated by electrostatic interactions between the positively charged PLL and the negatively charged polysaccharide cell structures. Dishes were incubated in $0,1 \mathrm{mg} / \mathrm{ml} P L L$ in deionized $\mathrm{H}_{2} \mathrm{O}$ for 30 min at $37^{\circ} \mathrm{C}$, washed twice with deionized $\mathrm{H}_{2} \mathrm{O}$, dried and used immediately.

\subsubsection{Immunocytochemistry}

Cells, grown on coverslips, were washed shortly in PBS, fixed in 4\% PFA for 10 min and washed again in PBS (3x). Permeabilization was performed for 1 min with $0,1 \%$ 
Triton X-100 in PBS at RT followed by blocking in 4\% horse serum in PBS for 30 min at RT. The primary antibody solution was prepared in $2 \%$ HS in PBS and added to the fixed cells for incubation over night at $4^{\circ} \mathrm{C}$ or for $1 \mathrm{~h}$ at RT. After washing in PBS (3x) cells were then incubated in the secondary antibody solution in $1,5 \%$ horse serum in PBS containing Dapi (1:20.000) or Topro (1:5000). After three more washing steps in PBS cells were dipped shortly in deionized $\mathrm{H}_{2} \mathrm{O}$ and mounted with Aqua Poly Mount.

\subsection{Microscopy and imaging}

\subsubsection{Electronmicroscopy}

A LEO EM912 Omega (Zeiss) with on-axis 2048 x 2048 CCD camera (Proscan) was used. Image acquisition was carried out by Wiebke Möbius and Boguslawa Sadowski.

\subsubsection{Brightfield microscopy}

Chromogenic stainings were analyzed with a Zeiss Axiophot microscope controlled by the Zen software and equipped with a AxioCamMRc3 camera. Single or tiled images were acquired with Plan-Neofluar 20x/0.50 objective or a EC Plan-Neofluar 40x/0.75 objective and subsequently stitched and corrected for shading inhomogeneities in the Zeiss Zen software. Images were exported in the .tiff image format and analyzed in FIJI.

\subsubsection{Epifluorescence microscopy}

\section{Setup}

A Zeiss Observer Z1 inverted microscope controlled by the Zen software and equipped with a 12-bit AxioCamMRm3 camera was used for the analysis of fluorescently labeled samples. A mercury arc lamp (HXP $120 \mathrm{~V}$ ) served as a light source that was filtered by the following filter cubes accommodated in a motorized filter turret. Dapi was visualized with a 365/50 $\mathrm{nm}$ band pass excitation filter, a $395 \mathrm{~nm}$ dichromatic mirror, and a 445/50 $\mathrm{nm}$ band-pass emission filter. EGFP, green mEos2, Cy2 and Alexa 488 were visualized with a $470 / 40 \mathrm{~nm}$ band pass excitation filter, a $495 \mathrm{~nm}$ dichromatic mirror, and a 525/50 $\mathrm{nm}$ band pass emission filter. Cy3, Alexa 555 and red mEos 2 were visualized with a $545 / 25 \mathrm{~nm}$ band pass excitation filter, a $570 \mathrm{~nm}$ dichromatic mirror, and a 605/70 nm band-pass emission filter. Cy5, Alexa 633 and Topro-3 were visualized with a 640/30 $\mathrm{nm}$ band pass excitation filter, a $660 \mathrm{~nm}$ dichromatic mirror, and a 690/50 $\mathrm{nm}$ bandpass emission filter. An adjustable rectangular diaphragm in the light path allowed the control over the size of the field of illumination. Cellular structures were visualized with a Plan-Neofluar 20x/0.50 objective and subcellular structures with a C-Apochromat 
63x/1.20 W Korr UV VIS IR objective. The microscope was equipped with an incubation chamber and controlled for temperature, $\mathrm{CO}_{2}$ and humidity.

\section{Image acquisition: Embedded samples}

For quantitative analysis of fluorescence signals the metal halide lamp was warmed up 20-30 min prior to use. The exposure was set manually for each staining such that at least $25 \%$ of the cameras dynamic range were covered. Samples that were to be compared were imaged on the same day and with the same parameters. For deconvolution 3D images were collected matching Nyquist criteria. Non-uniform illumination was corrected and shading correction performed in Zen before image export in the .czi format. For peroxisome quantification on brain vibratome sections a Z-stack spanning $3.2 \mu \mathrm{m}$ was acquired and analyzed in Imaris. For figures in section 3.3.1 and 3.3.2 a $6.4 \mu \mathrm{m}$ spanning Z-stack was acquired with subsequent deconvolution in the Zen program and final adjustment steps in Fiji. Pictures were analyzed in Fiji or Imaris.

\section{Image acquisition: Live-cell imaging}

Preincubation of the microscope was started 2-3 $\mathrm{h}$ before image acquisition to fully equilibrate the system and avoid drift. Temperature was kept at $37^{\circ} \mathrm{C}$ and air was humidified and enriched with $5 \% \mathrm{CO}_{2}$. A C-Apochromat 63x/1.20 W Korr UV VIS IR objective was used for all live cell experiments. To lower possible phototoxic effects exposure times were kept to a necessary minimum and 3D images were under sampled along the Z-axis with $0.5 \mu \mathrm{m}$ distance between each image. The rectangular diaphragm restricted the light reaching the sample to the field of view of the camera. Only a maximum of 20 positions per well were imaged to lower cell media exposition to light and thus generation of reactive oxygen species. A dark field picture was acquired and automatically subtracted from the final image to eliminate hot pixels. For photoconversion of mEos2 the rectangular diaphragm was set to either allow illumination of the whole cell or just a small region of interest and the sample was illuminated with ultraviolet light for $1200 \mathrm{~ms}$ at $100 \%$ lamp output. Green mEos 2 was imaged at $150 \mathrm{~ms}$ and red at 450 ms. Images were stitched, saved in the .czi format and further analyzed in Imaris.

\subsubsection{Nanoscopy}

Super resolution images by stimulated emission depletion (STED) were acquired on a homebuilt STED microscope equipped with a 63x glycerol immersion objective (Leica HCX PL APO 63x / 1.3) in collaboration with Katrin Willig. Details of the setup are described elsewhere (Willig et al., 2014). mEos was excited at $480 \mathrm{~nm}$ with a pulsed diode laser and 595nm STED light was provided by an optical parametric oscillator 
(OPO, APE, Berlin) pumped by a mode-locked Ti:Sapphire laser (Maitai, Spectra Physics) at a repetition rate of $80 \mathrm{MHz}$. The doughnut shape of the excitation beam was achieved by a vortex phase plate. PFA fixed brain sections were taken with a vibratome from Cnp-mEos2-PTS1 control and mutant mice and mounted in Aqua Poly mount. 5 stacks of images were acquired with a pixel size of $29 \mathrm{~nm}$ in $x$ and $y$ and a pixel dwell time of $20 \mu \mathrm{s}, 500 \mathrm{~nm}$ apart in $\mathrm{z}$. The resolution was $\leq 80 \mathrm{~nm}$.

\subsubsection{Magnetic resonance tomography}

Mice were primarily anesthetized with $5 \%$ Isoflurane and subsequently intubated and artificially ventilated with $1.75 \%$ Isoflurane. The animals were secured in a reproducible position and the body temperature was kept at $37^{\circ} \mathrm{C}$ by a heated water blanket. The resolution was $117 \mu \mathrm{m}$. All measurements were conducted by Dr. T. Michaelis in the Biodemedizinische NMR Forschungs $\mathrm{GmbH}$ at the Max-Planck Institute for biophysical chemistry.

\subsection{Image processing}

\subsubsection{Fiji}

Chromogenic images were quantified using the FIJI software. APP and CD3 stainings were counted manually with the multi point tool. The Mac3 positive area was quantified automatically after performing color deconvolution and thresholding.

In fluorescent images thresholding was performed with the auto local contrast function and fluorescently labeled areas were measured automatically. Calculations were performed in Microsoft Office Excel.

For publication a maximum intensity projection of deconvolved or undeconvovled Zstacks were conducted and fluorescence adjusted for better visualization.

\subsubsection{Imaris}

Peroxisome quantification was carried out in Imaris. 2D stacks were reduced to one plane by maximum intensity projection. The spots wizard was used to count peroxisomes and determine intraperoxisomal fluorescence. In Live-cell imaging experiments green and red peroxisomes were quantified automatically. Only green peroxisomes were calculated by the subtraction of red peroxisomes from green peroxisomes. For analysis of the peroxisomal size in images acquired by STED microscopy the Surfaces wizard was used with the region growing option enabled. The percentage of Olig2positive cells expressing mEos2 was determined by generation of mEos2-positve spots 
and Olig2-postive surfaces and colocalization of the same. In Cnp-mEos2-PTS1 transgenic animals crossbred to Cnp-Mfp2 mutants peroxisomes were quantified by the spots wizard. Oligodendrocytes were defined as Topro positive nuclei surrounded by mEos2 positive peroxisomes. Peroxisomes further than $4 \mu \mathrm{m}$ away from an oligodendrocyte nucleus were identified by the 'Spots close to surface' function and were defined as peripheral peroxisomes. Calculations were performed in Microsoft Office Excel.

\subsubsection{Zen2}

Deconvolution was conducted in the Zen Software. A fast iterative default mode was applied.

\subsection{Animals and behavior}

All mice used in this study were bred and maintained in the animal facility of the Max Planck Institute of Experimental Medicine. Animal maintenance, handling and experiments were in compliance with the German animal welfare laws and regulations. Best efforts were made to reduce animal suffering as well as animal numbers used for experiments. Food and tap water were available ad libitum. Mice were kept in standard cages (Makrolon Type2, $19 \times 34 \times 20 \mathrm{~cm}$ ). The room temperature was kept at $22 \pm 1^{\circ} \mathrm{C}$ and the $12 / 12 \mathrm{~h}$ day and night cycle was kept constant with light on from 4.30 am till $4.30 \mathrm{pm}$. Animals were sacrificed by cervical dislocation for fresh tissue collection or anesthetized by intraperitoneal injection of Avertin $(100 \mu \mathrm{l} / 10 \mathrm{~g}$ bodyweight) for perfusion.

\subsubsection{Behavior}

For the evaluation of the overall condition of the mutants, mice were observed for presence $(+)$ or absence $(-)$ of altered fur appearance, kyphosis, ataxia, tremor, passiveness and hindlimb paralysis. Fur appearance, tremor and passiveness could best be judged by the monitoring of cage behavior. Atactic gait was analyzed by observation of the hindlimbs while the mouse was moving on a flat surface. An overall score was calculated for each mouse.

For a more detailed analysis of ataxia the grid test and the beam test were conducted. For the grid test mice were placed on a metal grid with a spacing of $1 \mathrm{~cm}^{2}$ in between the rods, which measured $2 \mathrm{~mm}$ in diameter. Mouse movement was observed for a distance of about $80 \mathrm{~cm}$ and slips counted. The test was repeated three times for each animal. The beam test consists of a $1.5 \mathrm{~cm}$ broad and $55 \mathrm{~cm}$ long elevated beam that 
terminates in a hiding box. Mice were placed at the end of the rod and were observed until they reached the hiding box. The number of slips were assessed in three repeats per mice.

\subsubsection{Generation of transgenic Cnp-mEos2-PTS1 mice}

Purified transgenic DNA prepared as described in section 6.1.1 was used for microinjection into $\mathrm{C} 57 \mathrm{BL} / 6 \mathrm{~N}$ pronuclei. Microinjected oocytes were then transferred to pseudopregnant mice. The procedure was performed at the transgenic facility of the Max Planck Institute of Experimental Medicine. Tail biopsies from 3 week old offspring were used for DNA preparation and genotyping to identify potential founder mice.

\subsubsection{Generation of $\mathrm{Cnp}^{\mathrm{Cre} / \mathrm{Wt} *} \mathrm{Mfp}^{-/-}$mice}

Transgenic Mfp $2^{\text {loxP/loxP }}$ animals were a generous gift from Myriam Baes and have been described before (Verheijden et al. 2013). Generation of mice containing MFP2deficient peroxisomes in myelinating glia cells was achieved by cross-breeding $M f p 2^{\text {loxP/loxP }}$ to Cnp-Cre transgenic mice (Lappe-Siefke et al. 2003). Mfp $2^{f l o x / f l o x}$ mice were used as controls.

\subsubsection{Genotyping of transgenic mice}

Tail biopsies were taken at the age of 3 weeks and DNA was prepared as described in section 6.1.1. The genotype was determined by PCR containing mutant specific genotyping primers. All genotyping primers were synthesized at the 'DNA core facility' of the Max Planck Institute of Experimental Medicine. They were provided at $50 \mathrm{pM}$ concentration and diluted 1:5.

\section{Cnp-mEos2-PTS1 genotyping}

PCR reaction mix

$\begin{array}{ll}1 \mu \mathrm{l} & \text { DNA } \\ 0.4 \mu \mathrm{l} & \text { Genotyping forward primer } \\ 0.4 \mu \mathrm{l} & \text { Genotyping reverse primer } \\ 2 \mu \mathrm{l} & \text { dNTP mix }(2 \mathrm{mM}) \\ 4 \mu \mathrm{l} & 5 \times \text { buffer } \\ 0.1 \mu \mathrm{l} & \text { go Taq polymerase }(1 \mathrm{U} / \mu \mathrm{l}) \\ 13.1 \mu \mathrm{l} & \text { deionized } \mathrm{H}_{2} \mathrm{O}\end{array}$

PCR program

$\begin{array}{ll}1.95^{\circ} \mathrm{C} & 3 \mathrm{~min} \\ 2.60^{\circ} \mathrm{C} & 30 \mathrm{sec} \\ 3.72^{\circ} \mathrm{C} & 1.5 \mathrm{~min} \\ 4.95^{\circ} \mathrm{C} & 30 \mathrm{sec} \\ 5.50^{\circ} \mathrm{C} & 1 \mathrm{~min} \\ 6.72^{\circ} \mathrm{C} & 10 \mathrm{~min}\end{array}$

7. $4^{\circ} \mathrm{C} \quad$ pause

2 to $4: 34$ cycles 


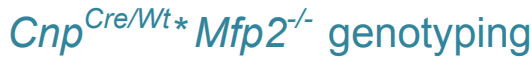

Mfp2 flox/flox genotyping

$\mathrm{PCR}$ reaction mix

$\begin{array}{ll}1 \mu \mathrm{l} & \text { DNA } \\ 0.5 \mu \mathrm{l} & \text { Genotyping forward primer } \\ 0.5 \mu \mathrm{l} & \text { Genotyping reverse primer } \\ 2 \mu \mathrm{l} & \text { dNTP mix }(2 \mathrm{mM}) \\ 4 \mu \mathrm{l} & 5 \times \text { buffer } \\ 0.1 \mu \mathrm{l} & \text { go Taq polymerase }(1 \mathrm{U} / \mu \mathrm{l}) \\ 12.9 \mu \mathrm{l} & \text { deionized } \mathrm{H}_{2} \mathrm{O}\end{array}$

PCR program

$\begin{array}{ll}1.95^{\circ} \mathrm{C} & 3 \mathrm{~min} \\ 2.65^{\circ} \mathrm{C} & 1 \mathrm{~min} \\ 3.72^{\circ} \mathrm{C} & 1 \mathrm{~min} \\ 4.95^{\circ} \mathrm{C} & 1 \mathrm{~min} \\ 5.65^{\circ} \mathrm{C} & 1 \mathrm{~min} \\ 6.72^{\circ} \mathrm{C} & 10 \mathrm{~min} \\ 7.4^{\circ} \mathrm{C} & \text { pause }\end{array}$

Step 2 to 4: 34

cycles

\section{Cnp-Cre genotyping}

$\mathrm{PCR}$ reaction mix
$1 \mu \mathrm{l} \quad$ DNA
$0.5 \mu \mathrm{l} \quad$ Genotyping forward primer
$0.25 \mu \mathrm{l} \quad$ Genotyping forward primer
$0.75 \mu \mathrm{l} \quad$ Genotyping reverse primer
$2 \mu \mathrm{l} \quad$ dNTP mix $(2 \mathrm{mM})$
$4 \mu \mathrm{l} \quad 5 \times$ buffer
$0.1 \mu \mathrm{l} \quad$ go Taq polymerase $(1 \mathrm{U} / \mu \mathrm{l})$
$12.4 \mu \mathrm{l} \quad$ deionized $\mathrm{H}_{2} \mathrm{O}$

$\begin{array}{ll}\text { PCR program } & \\ 1.95^{\circ} \mathrm{C} & 3 \mathrm{~min} \\ 2.50^{\circ} \mathrm{C} & 30 \mathrm{sec} \\ 3.72^{\circ} \mathrm{C} & 1 \mathrm{~min} \\ 4.95^{\circ} \mathrm{C} & 30 \mathrm{seC} \\ 5.50^{\circ} \mathrm{C} & 1 \mathrm{~min} \\ 6.72^{\circ} \mathrm{C} & 10 \mathrm{~min} \\ 7.4^{\circ} \mathrm{C} & \text { pause }\end{array}$

Step 2 to $4: 34$

cycles

\subsection{Data analysis}

For histological analysis the mean of the technical replicates of each sample was combined to one data point. $\mathrm{N}$-numbers reflect biological replicates. For live-cell imaging at least 10 cells per condition were analyzed and n-numbers reflect independent experiments. Data analysis was conducted in Microsoft Excel 2011. Data is presented as mean + standard deviation (SD). Statistical significance between two groups was determined by the unpaired Student's $t$ test assuming normal distribution between samples. P-values smaller than 0.05 were considered significant. P-values $<0.05$ are displayed with $\left({ }^{*}\right),<0.01$ with $\left({ }^{* *}\right)$ and $<0.001$ with $\left({ }^{* *}\right)$. 


\section{References}

Agrawal G, Joshi S, Subramani S (2011) Cell-free sorting of peroxisomal membrane proteins from the endoplasmic reticulum. Proc Natl Acad Sci USA 108:9113-9118

Ahlemeyer B, Neubert I, Kovacs WJ, Baumgart-Vogt E (2007) Differential expression of peroxisomal matrix and membrane proteins during postnatal development of mouse brain. J Comp Neurol 505:1-17

Alberts B, Johnson A, Lewis J, et al. Molecular Biology of the Cell. 4th edition (2002)

New York: Garland Science;. Peroxisomes. Available from:

http://www.ncbi.nlm.nih.gov/books/NBK26858/

Apanasets O, Grou CP, Van Veldhoven PP, Brees C, Wang B, Nordgren M, Dodt G, Azevedo JE, Fransen M (2013) PEX5, the Shuttling Import Receptor for Peroxisomal Matrix Proteins, Is a Redox-Sensitive Protein. Traffic:n/a-n/a

Aranovich A, Hua R, Rutenberg AD, Kim PK (2014) PEX16 contributes to peroxisome maintenance by constantly trafficking PEX3 via the ER. Journal of Cell Science 127:3675-3686

Baarine M, Andréoletti $P$, Athias A, Nury T, Zarrouk A, Ragot K, Vejux A, Riedinger JM, Kattan Z, Bessede G, Trompier D, Savary S, Cherkaoui-Malki M, Lizard G (2012) Evidence of oxidative stress in very long chain fatty acid â€" Treated oligodendrocytes and potentialization of ROS production using RNA interferencedirected knockdown of ABCD1 and ACOX1 peroxisomal proteins. Neuroscience 213:1-18

Baarine M, Ragot K, Athias A, Nury T, Kattan Z, Genin EC, Andreoletti P, Ménétrier F, Riedinger J-M, Bardou M, Lizard G (2012) Incidence of Abcd1 level on the induction of cell death and organelle dysfunctions triggered by very long chain fatty acids and TNF-Î \pm on oligodendrocytes and astrocytes. Neurotoxicology 33:212-228

Baes M, Huyghe S, Carmeliet P, Declercq PE, Collen D, Mannaerts GP, Van Veldho ven PP (2000) Inactivation of the peroxisomal multifunctional protein-2 in mice impedes the degradation of not only 2-methyl-branched fatty acids and bile acid intermediates but also of very long chain fatty acids. J Biol Chem 275:16329-16336

Baes M, Van Veldhoven PP (2012) Mouse models for peroxisome biogenesis defects and $\beta$-oxidation enzyme deficiencies. BBA - Molecular Basis of Disease 1822:1489-1500

Baes M, Gressens $P$, Baumgart E, Carmeliet $P$, Casteels $M$, Fransen M, Evrard $P$, Fahimi D, Declercq PE, Collen D, Van Veldhoven PP, Mannaerts GP (1997) A mouse model for Zellweger syndrome. Nat Genet 17:49-57

Ballabh P, Braun A, Nedergaard M (2004) The blood-brain barrier: an overview. Neurobiology of Disease 16:1-13

Barford D (2004) The role of cysteine residues as redox-sensitive regulatory switches. 
Current Opinion in Structural Biology 14:679-686

Baumann N, Pham-Dinh D (2001) Biology of oligodendrocyte and myelin in the mammalian central nervous system. Physiol Rev 81:871-927

Bechmann I, Nitsch R (1997) Astrocytes and microglial cells incorporate degenerating fibers following entorhinal lesion: a light, confocal, and electron microscopical study using a phagocytosis-dependent labeling technique. Glia 20:145-154

Belachew S, Yuan X, Gallo V (2001) Unraveling oligodendrocyte origin and function by cell-specific transgenesis. Dev Neurosci 23:287-298

Birnboim HC, and Doly J (1979) A rapid alkaline extraction procedure for screening recombinant plasmid DNA. Nucleic Acids Res 7(6): 1513-1523

Boiko T, Winckler B (2006) Myelin under construction -- teamwork required. The Journal of Cell Biology 172:799-801

Bonekamp NA, Grille S, Cardoso MJ, Almeida M, Aroso M, Gomes S, Magalhaes AC, Ribeiro D, Islinger M, Schrader M (2013) Self-Interaction of Human Pex11p $\beta$ during Peroxisomal Growth and Division (H Einwaechter, Ed.). PLoS ONE 8:e53424-16

Boveris A, Oshino N, Chance B (1972) The cellular production of hydrogen peroxide. Biochem J 128:617-630

Braverman NE, Moser AB (2012) Functions of plasmalogen lipids in health and disease. BBA - Molecular Basis of Disease 1822:1442-1452

Bright JJ, Natarajan C, Muthian G, Barak Y, Evans RM (2003) Peroxisome ProliferatorActivated Receptor- -Deficient Heterozygous Mice Develop an Exacerbated Neural Antigen-Induced Th1 Response and Experimental Allergic Encephalomyelitis. The Journal of Immunology 171:5743-5750

Brites P, Mooyer PAW, Mrabet el L, Waterham HR, Wanders RJA (2008) Plasmalogens participate in very-long-chain fatty acid-induced pathology. Brain 132:482492

Brocard C, Hartig A (2014) Molecular Machines Involved in Peroxisome Biogenesis and Maintenance. Springer

Brosius U, Dehmel T, Gartner J (2002) Two Different Targeting Signals Direct Human Peroxisomal Membrane Protein 22 to Peroxisomes. J Biol Chem 277:774-784

Brown GC, Neher JJ (2014) Microglial phagocytosis of live neurons. Nature Publishing Group 15:209-216

Burda JE, Sofroniew MV (2014) Reactive Gliosis and the Multicellular Response to CNS Damage and Disease. Neuron 81:229-248

Carvalho AF, Pinto MP, Grou CP, Alencastre IS, Fransen M, Sá-Miranda C, Azevedo JE (2007) Ubiquitination of mammalian Pex5p, the peroxisomal import receptor. J Biol Chem 282:31267-31272 
Chang CC, South S, Warren D, Jones J, Moser AB, Moser HW, Gould SJ (1999) Metabolic control of peroxisome abundance. Journal of Cell Science 112 ( Pt 10):1579-1590

Cheon J-E, Kim I-O, Hwang YS, Kim KJ, Wang K-C, Cho B-K, Chi JG, Kim CJ, Kim WS, Yeon KM (2002) Leukodystrophy in children: a pictorial review of MR imaging features. Radiographics 22:461-476

Colton HM (2004) Visualization and Quantitation of Peroxisomes Using Fluorescent Nanocrystals: Treatment of Rats and Monkeys with Fibrates and Detection in the Liver. Toxicological Sciences 80:183-192

Dansen TB, Wirtz KW, Wanders RJ, Pap EH (2000) Peroxisomes in human fibroblasts have a basic $\mathrm{pH}$. Nat Cell Biol 2:51-53

Davidoff MS, Middendorff R, Köfüncü E, Müller D, Ježek D, Holstein AF (2002a) Leydig cells of the human testis possess astrocyte and oligodendrocyte marker molecules. Acta Histochemica 104:39-49

Davidoff MS, Middendorff R, Köfüncü E, Müller D, Ježek D, Holstein AF (2002b) Leydig cells of the human testis possess astrocyte and oligodendrocyte marker molecules. Acta Histochemica 104:39-49

de Castro DCAF (2013) The effect of glia-glia interactions on oligodendrocyte precursor cell biology during development and in demyelinating diseases. :1-15

De Duve C, Baudhuin P (1966) Peroxisomes (microbodies and related particles). Physiol Rev 46:323-357

Delille HK, Agricola B, Guimaraes SC, Borta H, Luers GH, Fransen M, Schrader M (2010) Pex11p -mediated growth and division of mammalian peroxisomes follows a maturation pathway. Journal of Cell Science 123:2750-2762

Doetsch F, García-Verdugo JM, Alvarez-Buylla A (1997) Cellular composition and three-dimensional organization of the subventricular germinal zone in the adult mammalian brain. Journal of Neuroscience 17:5046-5061

Dreyer C, Krey G, Keller H, Givel F, Helftenbein G (1992) Control of the peroxisomal $\beta$ oxidation pathway by a novel family of nuclear hormone receptors. Cell 68:879887

Dyer JM, McNew JA, Goodman JM (1996) The sorting sequence of the peroxisomal integral membrane protein PMP47 is contained within a short hydrophilic loop. The Journal of Cell Biology 133:269-280

Eichler FS, Ren J-Q, Cossoy M, Rietsch AM, Nagpal S, Moser AB, Frosch MP, Ransohoff RM (2008) Is microglial apoptosis an early pathogenic change in cerebral X-linked adrenoleukodystrophy? Ann Neurol 63:729-742

Engelen M, Kemp S, de Visser M, van Geel BM, Wanders RJA, Aubourg P, Poll-The BT (2012) X-linked adrenoleukodystrophy (X-ALD): clinical presentation and guidelines for diagnosis, follow-up and management. Orphanet J Rare Dis 7:51

Evans MJ, Finean JB (1965) The lipid composition of myelin from brain and peripheral 
nerve. Journal of Neurochemistry 12:729-734

Fakieh MH, Drake PJM, Lacey J, Munck JM, Motley AM, Hettema EH (2013) Intra-ER sorting of the peroxisomal membrane protein Pex3 relies on its luminal domain. Biology Open 2:829-837

Fang Y, Morrell JC, Jones JM, Gould SJ (2004) PEX3 functions as a PEX19 docking factor in the import of class I peroxisomal membrane proteins. The Journal of Cell Biology 164:863-875

Farooqui AA, Horrocks LA, Farooqui T (2000) Glycerophospholipids in brain: their metabolism, incorporation into membranes, functions, and involvement in neurological disorders. Chemistry and Physics of Lipids 106:1-29

Feinstein DL, Galea E, Gavrilyuk V, Brosnan CF, Whitacre CC, Dumitrescu-Ozimek L, Landreth GE, Pershadsingh HA, Weinberg G, Heneka MT (2002) Peroxisome proliferator-activated receptor-? agonists prevent experimental autoimmune encephalomyelitis. Ann Neurol 51:694-702

Ferdinandusse S, Denis S, Hogenhout EM, Koster J, van Roermund CWT, IJlst L, Moser AB, Wanders RJA, Waterham HR (2007) Clinical, biochemical, and mutational spectrum of peroxisomal acyl-coenzyme A oxidase deficiency. Hum Mutat 28:904912

Ferdinandusse S, Denis S, Mooyer PAW, Dekker C, Duran M, Soorani-Lunsing RJ, Boltshauser E, Macaya A, Gärtner J, Majoie CBLM, Barth PG, Wanders RJA, PollThe BT (2005) Clinical and biochemical spectrum of D-bifunctional protein deficiency. Ann Neurol 59:92-104

Ferdinandusse S, Denis S, Mooyer PAW, Dekker C, Duran M, Soorani-Lunsing RJ, Boltshauser E, Macaya A, Gärtner J, Majoie CBLM, Barth PG, Wanders RJA, PollThe BT (2006) Clinical and biochemical spectrum of D-bifunctional protein deficiency. Ann Neurol 59:92-104

Forss-Petter S, Werner H, Berger J, Lassmann H, Molzer B, Schwab MH, Bernheimer $\mathrm{H}$, Zimmermann F, Nave KA (1997) Targeted inactivation of the X-linked adrenoleukodystrophy gene in mice. J Neurosci Res 50:829-843

Fourcade S, Ferrer I, Pujol A (2015) Oxidative stress, mitochondrial and proteostasis malfunction in adrenoleukodystrophy_A paradigm for axonal degeneration. Free Radical Biology and Medicine 88:18-29

Fransen M, Nordgren M, Wang B, Apanasets O (2012) Role of peroxisomes in ROS/RNS-metabolism: Implications for human disease. BBA - Molecular Basis of Disease 1822:1363-1373

Fujiki Y (2014) Peroxisome biogenesis in mammalian cells. Front physiol. :1-8

Funk CD (2001) Prostaglandins and leukotrienes: advances in eicosanoid biology. Science 294:1871-1875

Fünfschilling U, Supplie LM, Mahad D, Boretius S, Saab AS, Edgar J, Brinkmann BG, Kassmann CM, Tzvetanova ID, Möbius W, Diaz F, Meijer D, Suter U, Hamprecht B, Sereda MW, Moraes CT, Frahm J, Goebbels S, Nave K-A (2013) Glycolytic oli- 
godendrocytes maintain myelin and long-term axonal integrity. Nature 485:517521

Galino J, Ruiz M, Fourcade S, Schlüter A, López-Erauskin J, Guilera C, Jove M, Naudi A, García-Arumí E, Andreu AL, Starkov AA, Pamplona R, Ferrer I, Portero-Otin M, Pujol A (2011) Oxidative Damage Compromises Energy Metabolism in the Axonal Degeneration Mouse Model of X-Adrenoleukodystrophy. 15:2095-2107

Gallyas F (1979) Silver staining of myelin by means of physical development. Neurol Res 1(2): 203-9

Geuze HJ, Murk JL, Stroobants AK, Griffith JM, Kleijmeer MJ, Koster AJ, Verkleij AJ, Distel B, Tabak HF (2003) Involvement of the endoplasmic reticulum in peroxisome formation. Molecular Biology of the Cell 14:2900-2907

Gould SJ, Valle D (2000) Peroxisome biogenesis disorders: genetics and cell biology. Trends Genet 16:340-345

Gould SJ, Keller GA, Hosken N, Wilkinson J, Subramani S (1989) A conserved tripeptide sorts proteins to peroxisomes. The Journal of Cell Biology 108:1657-1664

Gravel M, Di Polo A, Valera PB, Braun PE (1998) Four-kilobase sequence of the mouse CNP gene directs spatial and temporal expression of lacZ in transgenic mice. J Neurosci Res 53:393-404

Griffiths I, Klugmann M, Anderson T, Yool D, Thomson C, Schwab MH, Schneider A, Zimmermann F, McCulloch M, Nadon N, Nave KA (1998) Axonal swellings and degeneration in mice lacking the major proteolipid of myelin. Science 280:1610 1613

Grou CP, Carvalho AF, Pinto MP, Alencastre IS, Rodrigues TA, Freitas MO, Francisco T, Sá-Miranda C, Azevedo JE (2008) The peroxisomal protein import machinery a case report of transient ubiquitination with a new flavor. Cell Mol Life Sci 66:254262

Grou CP, Francisco T, Rodrigues TA, Freitas MO, Pinto MP, Carvalho AF, Domingues P, Wood SA, Rodríguez-Borges JE, Sá-Miranda C, Fransen M, Azevedo JE (2012) Identification of Ubiquitin-specific Protease 9X (USP9X) as a Deubiquitinase Acting on Ubiquitin-Peroxin 5 (PEX5) Thioester Conjugate. J Biol Chem 287:1281512827

Hagemeyer N, Goebbels S, Papiol S, Kästner A, Hofer S, Begemann M, Gerwig UC, Boretius S, Wieser GL, Ronnenberg A, Gurvich A, Heckers SH, Frahm J, Nave KA, Ehrenreich $\mathrm{H}$ (2012) A myelin gene causative of a catatonia-depression syndrome upon aging. EMBO Mol Med 4:528-539

Hajra AK (1997) Dihydroxyacetone phosphate acyltransferase. Biochim Biophys Acta 1348:27-34

Hanisch U-K, Kettenmann H (2007) Microglia: active sensor and versatile effector cells in the normal and pathologic brain. Nat Neurosci 10:1387-1394

Hara-Kuge S, Fujiki Y (2008) The peroxin Pex14p is involved in LC3-dependent degradation of mammalian peroxisomes. Experimental Cell Research 314:3531-3541 
Hardeman D, van den Bosch H (1991) Localization of enzymes involved in glyceroether bond formation in rat liver. ... et Biophysica Acta (BBA)-Lipids and Lipid ... 1081:285-292

Heiland I, Erdmann R (2005) Biogenesis of peroxisomes. FEBS Journal 272:23622372

Hein S, Schonfeld P, Kahlert S, Reiser G (2008) Toxic effects of X-linked adrenoleukodystrophy-associated, very long chain fatty acids on glial cells and neurons from rat hippocampus in culture. Human Molecular Genetics 17:1750-1761

Heneka M, Landreth G (2007) PPARs in the brain. Biochimica et Biophysica Acta (BBA) - Molecular and Cell Biology of Lipids 1771:1031-1045

Hess R, Stäubli W, Riess W (1965) Nature of the hepatomegalic effect produced by ethyl-chlorophenoxy-isobutyrate in the rat. Nature

Hoepfner D, Schildknegt D, Braakman I, Philippsen P, Tabak HF (2005) Contribution of the Endoplasmic Reticulum to Peroxisome Formation. Cell 122:85-95

Hu J, Desai M (2014) Light control of peroxisome proliferation during Arabidopsis photomorphogenesis. Plant Signaling \& Behavior 3:801-803

Hua R, Kim PK (2015) Multiple paths to peroxisomes: Mechanism of peroxisome maintenance in mammals. BBA - Molecular Cell Research:1-11

Hulshagen L, Krysko O, Bottelbergs A, Huyghe S, Klein R, Van Veldhoven PP, De Deyn PP, D'Hooge R, Hartmann D, Baes M (2008) Absence of functional peroxisomes from mouse CNS causes dysmyelination and axon degeneration. J Neurosci 28:4015-4027

Huybrechts SJ, Van Veldhoven PP, Brees C, Mannaerts GP, Los GV, Fransen M (2009) Peroxisome Dynamics in Cultured Mammalian Cells. Traffic 10:1722-1733

Huyghe S, Schmalbruch H, Hulshagen L, Veldhoven PV, Baes M, Hartmann D (2006) Peroxisomal Multifunctional Protein-2 Deficiency Causes Motor Deficits and Glial Lesions in the Adult Central Nervous System. The American Journal of Pathology 168:1321-1334

Igarashi M, Schaumburg HH, Powers J, Kishmoto Y, Kolodny E, Suzuki K (1976) Fatty acid abnormality in adrenoleukodystrophy. Journal of Neurochemistry 26:851-860

Issemann I, Green S (1990) Activation of a member of the steroid hormone receptor superfamily by peroxisome proliferators. Nature 347:645-650

Ito D, Imai Y, Ohsawa K, Nakajima K, Fukuuchi Y, Kohsaka S (1998) Microglia-specific localisation of a novel calcium binding protein, Iba1. Brain Res Mol Brain Res $57: 1-9$

Itoyama A, Honsho M, Abe Y, Moser A, Yoshida Y, Fujiki Y (2012) Docosahexaenoic acid mediates peroxisomal elongation, a prerequisite for peroxisome division. Journal of Cell Science 125:589-602

Itoyama A, Michiyuki S, Honsho M, Yamamoto T, Moser A, Yoshida Y, Fujiki Y (2013) 
Mff functions with Pex11p and DLP1 in peroxisomal fission. Biology Open 2:9981006

Itzkovitz B, Jiralerspong S, Nimmo G, Loscalzo M, Horovitz DDG, Snowden A, Moser A, Steinberg S, Braverman N (2011) Functional characterization of novel mutations in GNPAT and AGPS, causing rhizomelic chondrodysplasia punctata (RCDP) types 2 and 3. Hum Mutat 33:189-197

Ivashchenko O, Van Veldhoven PP, Brees C, Ho Y-S, Terlecky SR, Fransen M (2011) Intraperoxisomal redox balance in mammalian cells: oxidative stress and interorganellar cross-talk. Molecular Biology of the Cell 22:1440-1451

Iwata J-I, Ezaki J, Komatsu M, Yokota S, Ueno T, Tanida I, Chiba T, Tanaka K, Kominami E (2006) Excess Peroxisomes Are Degraded by Autophagic Machinery in Mammals. J Biol Chem 281:4035-4041

Jankowski A, Kim JH, Collins RF, Daneman R, Walton P, Grinstein S (2001) In Situ Measurements of the $\mathrm{pH}$ of Mammalian Peroxisomes Using the Fluorescent Protein pHluorin. J Biol Chem 276:48748-48753

Jansen GA, Waterham HR, Wanders RJA (2004) Molecular basis of Refsum disease: Sequence variations in Phytanoyl-CoA Hydroxylase (PHYH) and the PTS2 receptor (PEX7). Hum Mutat 23:209-218

Jing K, Lim K (2012) Why is autophagy important in human diseases? Exp Mol Med 44:69-4

Joseph KC, Druse MJ, Newell LR, Hogan EL (1972) Fatty acid composition of cerebrosides, sulphatides and ceramides in murine leucodystrophy: the quaking mutant. Journal of Neurochemistry 19:307-312

Joshi S, Agrawal G, Subramani S (2012) Phosphorylation-dependent Pex11p and Fis $1 p$ interaction regulates peroxisome division. Molecular Biology of the Cell 23:1307-1315

Karbowski J (2007) Global and regional brain metabolic scaling and its functional consequences. BMC Biol 5:18-11

Kassmann CM, Lappe-Siefke C, Baes M, Brügger B, Mildner A, Werner HB, Natt O, Michaelis T, Prinz M, Frahm J, Nave K-A (2007) Axonal loss and neuroinflammation caused by peroxisome-deficient oligodendrocytes. Nat Genet 39:969-976

Kassmann CM, Quintes S, Rietdorf J, Möbius W, Sereda MW, Nientiedt T, Saher G, Baes M, Nave K-A (2011) A role for myelin-associated peroxisomes in maintaining paranodal loops and axonal integrity. FEBS LETTERS:1-7

Kaushik S, Massey AC, Mizushima N, Cuervo AM (2008) Constitutive Activation of Chaperone-mediated Autophagy in Cells with Impaired Macroautophagy. Molecular Biology of the Cell 19:2179-2192

Kiel JAKW, Veenhuis M, van der Klei IJ (2006) PEX Genes in Fungal Genomes: Common, Rare or Redundant. Traffic 7:1291-1303

Kikuchi M, Hatano N, Yokota S, Shimozawa N, Imanaka T, Taniguchi H (2004) Prote- 
omic Analysis of Rat Liver Peroxisome: PRESENCE OF PEROXISOME-SPECIFIC ISOZYME OF LON PROTEASE. J Biol Chem 279:421-428

Kim N, Jeong S, Jing K, Shin S, Kim S, Heo J-Y, Kweon G-R, Park S-K, Wu T, Park JI, Lim K (2015) Docosahexaenoic Acid Induces Cell Death in Human Non-Small Cell Lung Cancer Cells by Repressing mTOR via AMPK Activation and PI3K/Akt Inhibition. BioMed Research International 2015:1-14

Kim PK (2006) The origin and maintenance of mammalian peroxisomes involves a de novo PEX16-dependent pathway from the ER. The Journal of Cell Biology 173:521-532

Kim PK, Hailey DW, Mullen RT, Lippincott-Schwartz J (2008) Ubiquitin signals autophagic degradation of cytosolic proteins and peroxisomes. Proc Natl Acad Sci USA 105:20567-20574

Kliewer SA, Umesono K, Mangelsdorf DJ, Evans RM (1992) Retinoid X receptor interacts with nuclear receptors in retinoic acid, thyroid hormone and vitamin D3 signalling. Nature 355:446-449

Knoblach B, Rachubinski RA (2010) Phosphorylation-dependent Activation of Peroxisome Proliferator Protein PEX11 Controls Peroxisome Abundance. J Biol Chem 285:6670-6680

Kobayashi TEA (1997) Adrenoleukodystrophy Protein-Deficient Mice Represent Abnormality of Very Long Chain Fatty Acid Metabolism. :1-6

Koch J, Brocard C Membrane elongation factors in organelle maintenance: the case of peroxisome proliferation (2011). BioMolecular Concepts 2:353-364

Koga H, Kaushik S, Cuervo AM (2010) Altered lipid content inhibits autophagic vesicular fusion. The FASEB Journal 24:3052-3065

Kovacs WJ, Faust PL, Keller GA, Krisans SK (2001) Purification of brain peroxisomes and localization of 3-hydroxy-3-methylglutaryl coenzyme A reductase. Eur J Biochem 268:4850-4859

Lam SK, Yoda N, Schekman R (2011) PNAS Plus: A vesicle carrier that mediates peroxisome protein traffic from the endoplasmic reticulum. Proceedings of the National Academy of Sciences 108:E51-E52

Laplante M, Sabatini DM (2009) mTOR signaling at a glance. Journal of Cell Science 122:3589-3594

Lappe-Siefke C, Goebbels S, Gravel M, Nicksch E, Lee J, Braun PE, Griffiths IR, Nave K-A (2003) Disruption of Cnp1 uncouples oligodendroglial functions in axonal support and myelination. Nat Genet 33:366-374

Launay N, Aguado C, Fourcade S, Ruiz M, Grau L, Riera J, Guilera C, Giròs M, Ferrer I, Knecht E, Pujol A (2014) Autophagy induction halts axonal degeneration in a mouse model of X-adrenoleukodystrophy. Acta Neuropathol 129:399-415

Lazarow PB (1978) Rat liver peroxisomes catalyze the beta oxidation of fatty acids. J Biol Chem 253:1522-1528 
Lazarow PB, Fujiki Y (1985) Biogenesis of peroxisomes. Annu Rev Cell Biol 1:489-530

Lemay DG (2006) Genome-wide identification of peroxisome proliferator response elements using integrated computational genomics. The Journal of Lipid Research $47: 1583-1587$

Léon S, Goodman JM, Subramani S (2006) Uniqueness of the mechanism of protein import into the peroxisome matrix: Transport of folded, co-factor-bound and oligomeric proteins by shuttling receptors. Biochimica et Biophysica Acta (BBA) Molecular Cell Research 1763:1552-1564

Li X, Gould SJ (2002) PEX11 promotes peroxisome division independently of peroxisome metabolism. The Journal of Cell Biology 156:643-651

Li X, Baumgart E, Dong GX, Morrell JC, Jimenez-Sanchez G, Valle D, Smith KD, Gould SJ (2002) PEX11 Is Required for Peroxisome Proliferation in Response to 4-Phenylbutyrate but Is Dispensable for Peroxisome Proliferator-Activated Receptor Alpha-Mediated Peroxisome Proliferation. Molecular and Cellular Biology 22:8226-8240

Lieber DS, Hershman SG, Slate NG, Calvo SE, Sims KB, Schmahmann JD, Mootha VK (2014) Next generation sequencing with copy number variant detection expands the phenotypic spectrum of HSD17B4-deficiency. BMC Med Genet 15:30

Lieberman AP, Puertollano R, Raben N, Slaugenhaupt S, Walkley SU, Ballabio A (2012) Autophagy in lysosomal storage disorders. Autophagy 8:719-730

Lines MA, Jobling R, Brady L, Marshall CR, Scherer SW, Rodriguez AR, Lee L, Lang AE, Mestre TA, Wanders RJA, Ferdinandusse S, Tarnopolsky MA, Canadian Pediatric Genetic Disorders Sequencing Consortium (FORGE Canada) (2014) Peroxisomal D-bifunctional protein deficiency: three adults diagnosed by whole-exome sequencing. Neurology 82:963-968

Lu JF, Lawler AM, Watkins PA, Powers JM, Moser AB, Moser HW, Smith KD (1997) A mouse model for $X$-linked adrenoleukodystrophy. Proceedings of the National Academy of Sciences 94:9366-9371

Ma C, Hagstrom D, Polley SG, Subramani S (2013) Redox-regulated Cargo Binding and Release by the Peroxisomal Targeting Signal Receptor, Pex5. J Biol Chem 288:27220-27231

Manivannan S, de Boer R, Veenhuis M, van der Klei IJ (2014) Lumenal peroxisomal protein aggregates are removed by concerted fission and autophagy events. Autophagy 9:1044-1056

Mao K, Liu X, Feng Y, Klionsky DJ (2014) The progression of peroxisomal degradation through autophagy requires peroxisomal division. Autophagy 10:652-661

Marzioch M, Erdmann R, Veenhuis M, Kunau WH (1994) PAS7 encodes a novel yeast member of the WD-40 protein family essential for import of 3-oxoacyl-CoA thiolase, a PTS2-containing protein, into peroxisomes. The EMBO Journal 13:4908-4918

Matsuzono Y, Kinoshita N, Tamura S, Shimozawa N, Hamasaki M, Ghaedi K, Wanders RJ, Suzuki Y, Kondo N, Fujiki Y (1999) Human PEX19: cDNA cloning by functional 
complementation, mutation analysis in a patient with Zellweger syndrome, and potential role in peroxisomal membrane assembly. Proceedings of the National Academy of Sciences 96:2116-2121

Matthews MA, Duncan D (1971) A quantitative study of morphological changes accompanying the initiation and progress of myelin production in the dorsal funiculus of the rat spinal cord. J Comp Neurol 142:1-22

Mauch DH, Nägler K, Schumacher S, Göritz C, Müller EC, Otto A, Pfrieger FW (2001) CNS synaptogenesis promoted by glia-derived cholesterol. Science 294:13541357

McCarthy KD, de Vellis J (1980) Preparation of separate astroglial and oligodendroglial cell cultures from rat cerebral tissue. The Journal of Cell Biology 85:890-902

McKenna O, Arnold G, Holtzman E (1976) Microperoxisome distribution in the central nervous system of the rat. Brain Res 117:181-194

McKinney SA, Murphy CS, Hazelwood KL (2009) A bright and photostable photoconvertible fluorescent protein. Nature 6:131-133

McMullen PD, Bhattacharya S, Woods CG, Bin Sun, Yarborough K, Ross SM, Miller ME, McBride MT, LeCluyse EL, Clewell RA, Andersen ME (2014) A map of the PPARÎ \pm transcription regulatory network for primary human hepatocytes. CHEMICO-BIOLOGICAL INTERACTIONS 209:14-24

Meinecke M, Cizmowski C, Schliebs W, Krüger V, Beck S, Wagner R, Erdmann R (2010) The peroxisomal importomer constitutes a large and highly dynamic pore. Nature Publishing Group 12:273-277

Michalik L, Auwerx J, Berger JP, Chatterjee VK, Glass CK, Gonzalez FJ, Grimaldi PA, Kadowaki T, Lazar MA, O'Rahilly S, Palmer CNA, Plutzky J, Reddy JK, Spiegelman BM, Staels B, Wahli W (2006) International Union of Pharmacology. LXI. Peroxisome Proliferator-Activated Receptors. Pharmacological Reviews 58:726-741

Montilla-Martinez M, Beck S, Klümper J, Meinecke M, Schliebs W, Wagner R, Erdmann R (2015) Distinct Pores for Peroxisomal Import of PTS1 and PTS2 Proteins. CellReports 13:2126-2134

Moser HW, Moser AB, Smith KD, Bergin A, Borel J, Shankroff J, Stine OC, Merette C, Ott J, Krivit W, Shapiro E (1992) Adrenoleukodystrophy: Phenotypic variability and implications for therapy. J Inherit Metab Dis 15:645-664

Mullis K, Faloona F, Scharf S, Saiki R, Horn G, Erlich H (1986), Cold Spring Harb Symp Quant Biol., 51(1), 263

Nimmerjahn A, Kirchhoff F, Helmchen F (2005) Resting microglial cells are highly dynamic surveillants of brain parenchyma in vivo. Science 308:1314-1318

Nixon RA (2013) The role of autophagy in neurodegenerative disease. Nat Med 19:983-997 
Nordgren M, Francisco T, Lismont C, Hennebel L, Brees C, Wang B, Van Veldhoven PP, Azevedo JE, Fransen M (2015) Export-deficient monoubiquitinated PEX5 triggers peroxisome removal in SV40 large T antigen-transformed mouse embryonic fibroblasts. Autophagy 11:1326-1340

Novikoff AB, Novikoff PM, Davis C, Quintana N (1972) Studies on microperoxisomes. II. A cytochemical method for light and electron microscopy. J Histochem Cytochem 20:1006-1023

Braissant O, Foufelle F, Scotto C, Dauça M, Wahli W (1996) Differential expression of peroxisome proliferator-activated receptors (PPARs): tissue distribution of PPARalpha, -beta, and -gamma in the adult rat. Endocrinology 137:354-366

O'Brien JS, Sampson EL (1965) Lipid composition of the normal human brain: gray matter, white matter, and myelin. The Journal of Lipid Research 6:537-544

Ofman R, Hettema EH, Hogenhout EM (1998) Acyl-CoA: dihydroxyacetonephosphate acyltransferase: cloning of the human cDNA and resolution of the molecular basis in rhizomelic chondrodysplasia punctata .... Human molecular ...

Pekny M, Pekna M (2004) Astrocyte intermediate filaments in CNS pathologies and regeneration (FC Ramaekers and FT Bosman, Eds.). J Pathol 204:428-437

Pellerin L, Bouzier-Sore A-K, Aubert A, Serres S, Merle M, Costalat R, Magistretti PJ (2007) Activity-dependent regulation of energy metabolism by astrocytes: An update. Glia 55:1251-1262

Peters JM, Cheung C, Gonzalez FJ (2005) Peroxisome proliferator-activated receptor$\alpha$ and liver cancer: where do we stand? J Mol Med 83:774-785

Peters JM, Lee SS, Li W, Ward JM, Gavrilova O, Everett C, Reitman ML, Hudson LD, Gonzalez FJ (2000) Growth, adipose, brain, and skin alterations resulting from targeted disruption of the mouse peroxisome proliferator-activated receptor beta(delta). Molecular and Cellular Biology 20:5119-5128

Pillai BK, Jasuja R, Simard JR, Hamilton JA (2009) Fast Diffusion of Very Long Chain Saturated Fatty Acidsacross a Bilayer Membrane and Their Rapid Extractionby Cyclodextrins. J Biol Chem 284:1-9

Pinto MP, Grou CP, Fransen M, Sá-Miranda C, Azevedo JE (2009) The cytosolic domain of PEX3, a protein involved in the biogenesis of peroxisomes, binds membrane lipids. BBA - Molecular Cell Research 1793:1669-1675

Poll-The BT, Roels F, Ogier H, Scotto J, Vamecq J, Schutgens RB, Wanders RJ, van Roermund CW, van Wijland MJ, Schram AW (1988) A new peroxisomal disorder with enlarged peroxisomes and a specific deficiency of acyl-CoA oxidase (pseudoneonatal adrenoleukodystrophy). Am J Hum Genet 42:422-434

Powers JM, Liu Y, Moser AB, Moser HW (1992) The inflammatory myelinopathy of adreno-leukodystrophy: cells, effector molecules, and pathogenetic implications. J Neuropathol Exp Neurol 51:630-643 
Pujol A, Hindelang C, Callizot N, Bartsch U, Schachner M, Mandel J-L (2002) Late onset neurological phenotype of the X-ALD gene inactivation in mice: a mouse model for adrenomyeloneuropathy. Human Molecular Genetics 11:499-505

Rachubinski VITARA (2014) Origin and spatiotemporal dynamics of the peroxisomal endomembrane system. :1-2

Rakhshandehroo M, Knoch B, Müller M, Kersten S (2010) Peroxisome ProliferatorActivated Receptor Alpha Target Genes. PPAR Research 2010:1-20

Rhodin J (1954) Correlation of structural organization and function in normal and experimentally changed proximal convoluted tubule cells of the mouse kidney. Stockholm, Karolinska Inst, Dept of Anatomy

Richert S, Kleinecke S, Günther J, Schaumburg F, Edgar J, Nienhaus GU, Nave K-A, Kassmann CM (2014) In\&nbsp;vivo labeling of peroxisomes by photoconvertible mEos2 in myelinating glia of mice. Biochimie 98:127-134

Rosewich H (2005) Genetic and clinical aspects of Zellweger spectrum patients with PEX1 mutations. Journal of Medical Genetics 42:e58-e58

Rottensteiner H, Stein K, Sonnenhol E, Erdmann R (2003) Conserved function of pex $11 p$ and the novel pex25p and pex27p in peroxisome biogenesis. Molecular Biology of the Cell 14:4316-4328

Saab AS, Tzvetanova ID, Nave K-A (2013) ScienceDirect The role of myelin and oligodendrocytes in axonal energy metabolism. Current Opinion in Neurobiology 23:1065-1072

Sacksteder KA, Jones JM, South ST, Li X, Liu Y, Gould SJ (2000) PEX19 binds multiple peroxisomal membrane proteins, is predominantly cytoplasmic, and is required for peroxisome membrane synthesis. The Journal of Cell Biology 148:931-944

Saluja I, Granneman JG, Skoff RP (2001) PPAR $\delta$ agonists stimulate oligodendrocyte differentiation in tissue culture. Glia

Scherer SS, Braun PE, Grinspan J, Collarini E, Wang DY, Kamholz J (1994) Differential regulation of the 2',3'-cyclic nucleotide 3'-phosphodiesterase gene during oligodendrocyte development. Neuron 12:1363-1375

Scherz-Shouval R, Elazar Z (2011) Regulation of autophagy by ROS: physiology and pathology. Trends in Biochemical Sciences 36:30-38

Schönfeld P, Reiser G (2016) Brain Lipotoxicity of Phytanic Acid and Very Long-chain Fatty Acids. Harmful Cellular/Mitochondrial Activities in Refsum Disease and XLinked Adrenoleukodystrophy. A\&D 7:136-14

Schueren F, Lingner T, George R, Hofhuis J, Dickel C, Gärtner J, Thoms S (2014) Peroxisomal lactate dehydrogenase is generated by translational readthrough in mammals. eLife 3

Schuldiner M, Metz J, Schmid V, Denic V, Rakwalska M, Schmitt HD, Schwappach B, Weissman JS (2008) The GET Complex Mediates Insertion of Tail-Anchored Proteins into the ER Membrane. Cell 134:634-645 
Shaid S, Brandts $\mathrm{CH}$, Serve H, Dikic I (2012) Ubiquitination and selective autophagy. Cell Death and Differentiation 20:21-30

Shibata H, Kashiwayama Y, Imanaka T, Kato H (2004) Domain Architecture and Activity of Human Pex19p, a Chaperone-like Protein for Intracellular Trafficking of Peroxisomal Membrane Proteins. J Biol Chem 279:38486-38494

Simons M, Nave K-A (2016) Oligodendrocytes: Myelination and Axonal Support. Cold Spring Harb Perspect Biol 8:a020479-16

Smith JJ, Aitchison JD (2013) nrm3700. Nature Publishing Group 14:803-817

Sokoloff L (1977) Relation between physiological function and energy metabolism in the central nervous system. Journal of Neurochemistry 29:13-26

South ST, Sacksteder KA, Li X, Liu Y, Gould SJ (2000) Inhibitors of COPI and COPII do not block PEX3-mediated peroxisome synthesis. The Journal of Cell Biology 149:1345-1360

Sprangers J, Rabouille C (2015) SEC16 in COPII coat dynamics at ER exit sites. Biochm Soc Trans 43:97-103

Sprinkle TJ, McMorris FA, Yoshino J, DeVries GH (1985) Differential expression of 2':3'-cyclic nucleotide 3'-phosphodiesterase in cultured central, peripheral, and extraneural cells. Neurochem Res 10:919-931

Steelman AJ, Thompson JP, Li J (2012) Demyelination and remyelination in anatomically distinct regions of the corpus callosum following cuprizone intoxication. Neuroscience Research 72:32-42

Stoffel W, Bosio A (1997) Myelin glycolipids and their functions. Current Opinion in Neurobiology 7:654-661

Suzuki K, Ohsumi Y (2007) Molecular machinery of autophagosome formation in yeast, Saccharomyces cerevisiae. FEBS LETTERS 581:2156-2161

Suzuki T, Sakata H, Kato C, Connor JA, Morita M (2012) Astrocyte activation and wound healing in intact-skull mouse after focal brain injury. Eur J Neurosci 36:3653-3664

Swinkels BW, Gould SJ, Bodnar AG, Rachubinski RA, Subramani S (1991) A novel, cleavable peroxisomal targeting signal at the amino-terminus of the rat 3-ketoacylCoA thiolase. The EMBO Journal 10:3255-3262

Tanaka AR, Tanabe K, Morita M, Kurisu M, Kasiwayama Y, Matsuo M, Kioka N, Amachi T, Imanaka T, Ueda K (2002) ATP Binding/Hydrolysis by and Phosphorylation of Peroxisomal ATP-binding Cassette Proteins PMP70 (ABCD3) and Adrenoleukodystrophy Protein (ABCD1). J Biol Chem 277:40142-40147

Theodoulou FL, Holdsworth M, Baker A (2006) Peroxisomal ABC transporters. FEBS LETTERS 580:1139-1155

Thomas SE, Byers DM, Palmer F (1990) Incorporation of polyunsaturated fatty acids into plasmalogens, compared to other phospholipids of cultured glioma cells, is 
more dependent on chain length than on ....... et Biophysica Acta (BBA ...

Thoms S, Harms I, Kalies K-U, Gärtner J (2012) Peroxisome Formation Requires the Endoplasmic Reticulum Channel Protein Sec61. Traffic 13:599-609

Titorenko VI, Chan H, Rachubinski RA (2000) Fusion of small peroxisomal vesicles in vitro reconstructs an early step in the in vivo multistep peroxisome assembly pathway of Yarrowia lipolytica. The Journal of Cell Biology 148:29-44

Toro AA, Araya CA, Córdova GJ, Arredondo CA, Cárdenas HG, Moreno RE, Venegas A, Koenig CS, Cancino J, Gonzalez A, Santos MJ (2009) Pex3p-dependent peroxisomal biogenesis initiates in the endoplasmic reticulum of human fibroblasts. $J$ Cell Biochem 107:1083-1096

Troffer-Charlier N, Doerflinger N, Metzger E, Fouquet F, Mandel J-L, Aubourg P (1998) Mirror expression of adrenoleukodystrophy and adrenoleukodystrophy related genes in mouse tissues and human cell lines. European Journal of Cell Biology 75:254-264

Trompier D, Vejux A, Zarrouk A, Gondcaille C, Geillon F, Nury T, Savary S, Lizard G (2013) Brain peroxisomes. Biochimie:1-9

van der Knaap MS, Wassmer E, Wolf NI, Ferreira P, Topçu M, Wanders RJA, Waterham HR, Ferdinandusse $S$ (2012) MRI as diagnostic tool in early-onset peroxisomal disorders. Neurology 78:1304-1308

van der Zand A, Braakman I, Tabak HF (2010) Peroxisomal membrane proteins insert into the endoplasmic reticulum. Molecular Biology of the Cell 21:2057-2065

van der Zand A, Gent J, Braakman I, Tabak HF (2012) Biochemically Distinct Vesicles from the Endoplasmic Reticulum Fuse to Form Peroxisomes. Cell 149:397-409

Verheijden S, Bottelbergs A, Krysko O, Krysko DV, Beckers L, De Munter S, Van Veldhoven PP, Wyns S, Kulik W, Nave K-A, Ramer MS, Carmeliet P, Kassmann CM, Baes M (2013) Peroxisomal multifunctional protein-2 deficiency causes neuroinflammation and degeneration of Purkinje cells independent of very long chain fatty acid accumulation. Neurobiology of Disease:1-12

Wanders RJ, Waterham HR (2006) Biochemistry of mammalian peroxisomes revisited. Annu Rev Biochem 75:295-332

Wanders RJA (2014) Metabolic functions of peroxisomes in health and disease. Biochimie 98:36-44

Waterham HR, Ferdinandusse S, Wanders RJA (2016) Human disorders of peroxisome metabolism and biogenesis. BBA - Molecular Cell Research 1863:922-933

Weissbarth S, Maker HS, Raes I, Brannan TS, Lapin EP, Lehrer GM (1981) The activity of 2',3'-cyclic nucleotide 3'-phosphodiesterase in rat tissues. Journal of Neurochemistry 37:677-680

Wierzbicki AS (2007) Peroxisomal disorders affecting phytanic acid alpha-oxidation: a review. Biochm Soc Trans 35:881-886 
Wiesinger C, Kunze M, Regelsberger G, Forss-Petter S, Berger J (2013) Impaired Very Long-Chain Acyl-CoA -Oxidation in Human X-ALD Fibroblasts Is a Direct Consequence of $A B C D 1$ Transporter Dysfunction.

Williams C, van der Klei IJ (2014) The Functions of Pex11 Family Proteins in Peroxisome Biology. In: Molecular Machines Involved in Peroxisome Biogenesis and Maintenance. Springer Vienna, Vienna, p 425-437

Willig KI, Steffens H, Gregor C, Herholt A, Rossner MJ, Hell SW (2014) Nanoscopy of Filamentous Actin in Cortical Dendrites of a Living Mouse. Biophysj 106:L01-L03

Xiao Y, Karnati S, Qian G, Nenicu A, Fan W, Tchatalbachev S, Höland A, Hossain H, Guillou F, Lüers GH, Baumgart-Vogt E (2013) Cre -Mediated Stress Affects Sirtuin Expression Levels, Peroxisome Biogenesis and Metabolism, Antioxidant and Proinflammatory Signaling Pathways. PLoS ONE 7:e41097

Yamasaki M, Hashiguchi N, Fujiwara C, Imanaka T, Tsukamoto T, Osumi T (1999) Formation of peroxisomes from peroxisomal ghosts in a peroxisome-deficient mammalian cell mutant upon complementation by protein microinjection. J Biol Chem 274:35293-35296

Yanagihara T, Cumings JN (1969) Alterations of phospholipids, particularly plasmalogens, in the demyelination of multiple sclerosis as compared with that of cerebral oedema. Brain 92:59-70

Yokota S (2003) Degradation of normal and proliferated peroxisomes in rat hepatocytes: Regulation of peroxisomes quantity in cells. Microsc Res Tech 61:151160

Young K, O Rourke M, Gasperini R (2014) Adult myelination: wrapping up neuronal plasticity. Neural Regen Res 9:1261-4

Zhang J, Kim J, Alexander A, Cai S, Tripathi DN, Dere R, Tee AR, Tait-Mulder J, Di Nardo A, Han JM, Kwiatkowski E, Dunlop EA, Dodd KM, Folkerth RD, Faust PL, Kastan MB, Sahin M, Walker CL (2013) ncb2822. Nature 15:1186-1196

Zhang J, Tripathi DN, Jing J, Alexander A, Kim J, Powell RT, Dere R, Tait-Mulder J, Lee J-H, Paull TT, Pandita RK, Charaka VK, Pandita TK, Kastan MB, Walker CL (2015) ATM functions at the peroxisome to induce pexophagy in response to ROS. Nat Cell Biol 17:1259-1269

Zhao J, Dong J-N, Wang H-G, Zhao M, Sun J, Zhu W-M, Zuo L-G, Gong J-F, Li Y, Gu L-L, Li N, Li J-S (2015) Docosahexaenoic Acid Attenuated Experimental Chronic Colitis in Interleukin 10-Deficient Mice by Enhancing Autophagy Through Inhibition of the mTOR Pathway. Journal of Parenteral and Enteral Nutrition:1-6 


\section{Publications}

Richert S, Kleinecke S, Günther J, Schaumburg F, Edgar J, Nienhaus GU, Nave K-A, Kassmann CM (2014) In vivo labeling of peroxisomes by photoconvertible mEos2 in myelinating glia of mice. Biochimie 98:127-134

Mayerl S, Müller J, Bauer R, Richert S, Kassmann CM, Darras VM, Buder K, Boelen A, Visser TJ, Heuer H (2014) Transporters MCT8 and OATP1C1 maintain murine brain thyroid hormone homeostasis. J Clin Invest 124:1987-1999

Richert S, Nienhaus GU, Brügger B, Kleinecke S, Djbay P, Möbius W, Willig K, Verheijden $S$, Nave KA, Baes $M$, and Kassmann $C$. 'Central nervous system degeneration caused by lack of peroxisomal $\beta$-oxidation in oligodendrocytes' in preperation.

Kleinecke S, Richert S, de Hoz L, Quintes S, Blanz J, Naseri K, McGonigal R, Asadollahi E, Willison H, Brügger B, Sachsenheimer T, Wozny K, Möbius W, Baes M, Nave $\mathrm{KA}$, and Kassmann CM 'Conduction failure of myelinated axons caused by perturbed lipid metabolism in Schwann cells' in preperation. 
